hep-th/9902098

IFT-P.012/99

HUTP-99/A004

IASSNS-HEP-99-5

\title{
Conformal Field Theory Of AdS Background With Ramond-Ramond Flux
}

\author{
Nathan Berkovits \\ Instituto de Física Teórica, Universidade Estadual Paulista \\ Rua Pamplona 145, 01405-900, São Paulo, SP, Brasil \\ Cumrun Vafa \\ Jefferson Laboratory of Physics \\ Harvard University, Cambridge, MA 02138, USA \\ Edward Witten \\ School of Natural Sciences, Institute for Advanced Study \\ Olden Lane, Princeton, NJ 08540, USA
}

We review a formalism of superstring quantization with manifest six-dimensional spacetime supersymmetry, and apply it to $\mathrm{AdS}_{3} \times \mathbf{S}^{3}$ backgrounds with Ramond-Ramond flux. The resulting description is a conformal field theory based on a sigma model whose target space is a certain supergroup $S U^{\prime}(2 \mid 2)$.

February, 1999 


\section{Introduction}

In the RNS formulation of superstring theory, powerful conformal field theory methods of computation are available. A price one pays is that spacetime supersymmetry is not visible as a classical symmetry of the worldsheet action. It can be seen by incorporating spin fields and picture changing [1]. On the other hand, the Green-Schwarz description [2] makes spacetime supersymmetry manifest, but quantization becomes difficult, except in light cone gauge.

Though a fully covariant quantization of the Green-Schwarz string is not known, one does know a partial substitute in that it is possible to reformulate the RNS description in terms of Green-Schwarz-like variables, in a way that makes manifest a portion of the spacetime symmetry group. For example [3], one can exhibit four-dimensional Poincaré invariance with manifest $N=1$ supersymmetry (or, for Type II superstrings, $N=1$ from left-movers and $N=1$ from right-movers), giving a framework in which one can study superstring compactification on a Calabi-Yau manifold, with all symmetries more or less manifest. By a further extension of these ideas [4], one can exhibit six-dimensional $N=1$ super-Poincaré invariance (or $N=1$ for left-movers and $N=1$ for right-movers), giving a framework in which all symmetries of superstring compactification on a K3 surface are visible. These constructions are hybrids in which the spacetime symmetries of four or six of the ten dimensions are described in Green-Schwarz-like variables, while the remaining dimensions are described using RNS variables. These constructions may thus loosely, but only loosely, be described as covariant quantization of the Green-Schwarz string in four or six dimensions. There also exists a quantization of the ten-dimensional superstring which, after Wick-rotating, preserves manifest $U(5)$ super-Poincaré invariance [5].

One important property of the RNS approach to Type II superstrings is that in the RNS case, some of the bosonic fields - those from the Ramond-Ramond sector - are represented by spin fields. As a result, it is exceedingly difficult to describe and understand backgrounds in which Ramond-Ramond or RR fields are present in the vacuum. At best, one would have to describe such backgrounds by worldsheet Lagrangians with spin fields added to the action, leading at least generically to rather untransparent formulas. In addition, there are several questions of principle. A narrow one is simply that one must ask in which picture or pictures one should take the Ramond-Ramond vertex operators that are added to the Lagrangian. A broader question is this: What is supposed to replace worldsheet superconformal symmetry? Superconformal invariance is usually understood 
as the guiding principle of the RNS description of perturbative superstrings. However, in the presence of RR fields, the superconformal symmetry is violated (as the worldsheet supercurrents are not local with respect to a Ramond-Ramond field), and it is not at all clear even in principle what would constitute a satisfactory worldsheet action with RR fields.

The partially covariant methods mentioned before give an answer in principle, at least in certain special cases. If the RR fields live only in the four or six dimensions whose spacetime symmetries are manifest in the constructions of [3] or [4], then their vertex operators are ordinary untwisted worldsheet operators and can be added to the Lagrangian while sticking within a known framework in which the rules are all clear. For example, to leading order in an $\alpha^{\prime}$ expansion, a sigma model containing four-dimensional $\mathrm{RR}$ fields has been constructed in [6]. Although explicit $\beta$-function computations have not yet been done for this sigma model representing the Type II superstring, there have been $\beta$-function computations [7] in $D=4$ superspace for a closely related sigma model representing the heterotic superstring. The analogous sigma model construction has not been made in six dimensions either for the heterotic or Type II superstring.

The problem of describing conformal field theories with RR fields has acquired greater currency because of the much-studied dualities [8] between string theories on Anti de Sitter or AdS backgrounds that contain RR fields and certain conformal field theories. In the context of these dualities, it would be highly desireable to be able to construct conformal field theories representing backgrounds with RR fields. In the absence of an ability to do so, one is limited to studying certain limits of AdS theories in which supergravity is an adequate approximation.

The purpose of the present paper is to use the covariant Green-Schwarz-like methods mentioned above to construct a conformal field theory description of a particular AdS background with RR fields. This will be the Type IIB compactification on $\mathrm{AdS}_{3} \times \mathbf{S}^{3} \times \mathrm{K} 3$ $\left(\right.$ or $\left.\mathrm{AdS}_{3} \times \mathbf{S}^{3} \times \mathbf{T}^{4}\right)$. In fact, we will study the $\mathrm{AdS}_{3} \times \mathbf{S}^{3}$ examples with a general mixture of RR and Neveu-Schwarz fluxes. Though the details are complicated, the general outline of the answer that we will get is easily stated. $\mathrm{AdS}_{3} \times \mathbf{S}^{3}$ will be represented by a sigma model whose target space is a certain supergroup that we call $S U^{\prime}(2 \mid 2)$; this sigma model is conformally invariant because of special properties of that supergroup. The sigma model with target space $S U^{\prime}(2 \mid 2)$ depends on two parameters, which correspond physically to the RR and NS fluxes. For any values of the fluxes, this sigma model is highly nonunitary, because for example the worldsheet fermions have a second order kinetic energy; physical 
states will be those obeying a certain fairly elaborate set of constraints. Except in the special case that the RR flux vanishes, the $S U^{\prime}(2 \mid 2)$ sigma model must be supplemented with couplings of matter fields to ghost-like fields.

We should mention other approaches that give somewhat complementary information. The $\mathrm{AdS}_{3} \times \mathbf{S}^{3}$ models have cousins with NS fields [9] that can be described as conformal field theories using RNS variables [10,11]. One important ingredient in this approach is the $S L(2, \mathbf{R})$ WZW model, which has been studied from many points of view (for example, see [12]). Also, there are extremely elegant descriptions of AdS backgrounds with RR fields, including $\mathrm{AdS}_{3} \times \mathbf{S}^{3}$, at the classical level (for example, see [13]). Those descriptions involve strings in superspace, usually a superspace with more odd variables than will appear in our discussion, and with compensating $\kappa$ symmetry. There have also been attempts at $\mathrm{AdS}_{3} \times \mathbf{S}^{3}$ quantization in light cone and temporal gauges [14].

This paper is organized as follows. Because the methods we will use are comparatively little-known, we begin in sections 2-5 with an unusually detailed review and summary of previous work. The reader should not assume that everything in these sections (especially sections 2 and 3 ) is needed for understanding the rest of the paper. In section 6, we begin with $\mathbf{R}^{6} \times \mathrm{K} 3$ (or $\mathbf{R}^{6} \times \mathbf{T}^{4}$ ) and describe vertex operators that, locally and to first order, deform $\mathbf{R}^{6}$ to $\mathrm{AdS}_{3} \times \mathbf{S}^{3}$ with RR fields. Many general lessons become clear from this linearized treatment. Using those lessons, we construct in section 7 the conformal field theory - as mentioned before, a sigma model with target a certain supergroup - that describes $\mathrm{AdS}_{3} \times \mathbf{S}^{3}$. Then in section 8 , we analyze the necesssary ghost couplings and show their consistency with the framework reviewed in sections 2-5. In section 9, we define the conditions which physical vertex operators have to satisfy in the conformal field theory. In section 10, we study the $\mathrm{AdS}_{3} \times \mathbf{S}^{3}$ models with NS background in the same framework. Though this is "unnecessary," in the sense that conformal field theory descriptions of these backgrounds are already known, our approach has the virtue of making spacetime supersymmetry manifest - at the cost, we must admit, of a certain complexity.

There is one point of physics that we should perhaps mention here. One important route to the $\mathrm{AdS}_{3} \times \mathbf{S}^{3}$ backgrounds with RR flux begins with $\mathbf{R}^{6} \times \mathrm{K} 3\left(\right.$ or $\mathbf{R}^{6} \times \mathbf{T}^{4}$ ) with parallel Dirichlet onebranes and fivebranes in $\mathbf{R}^{6}$ (the fivebranes being wrapped on K3 or $\mathbf{T}^{4}$ to make strings in $\mathbf{R}^{6}$ ). Let $Q_{1}$ and $Q_{5}$ be the number of such strings or fivebranes. (Physically, one can also add threebranes wrapped on two-cycles in K3 or $\mathbf{T}^{4}$ to make additional strings, but in this case the methods we will use are much less effective, as the resulting RR flux does not "live" just in $\mathbf{R}^{6}$.) These integer variables determine the 
amount of RR flux on the $\mathbf{S}^{3}$ factor in $\mathrm{AdS}_{3} \times \mathbf{S}^{3}$, and also the volume of the $\mathrm{K} 3$ or $\mathbf{T}^{4}$. In our work, however, we will not see any quantization of either of those variables. One way to explain the reason is as follows. Let $\lambda$ be the string coupling constant. The number of RR flux quanta needed to make an $\mathrm{AdS}_{3} \times \mathbf{S}^{3}$ background that remains fixed as $\lambda \rightarrow 0$ is of order $1 / \lambda$. Since (by doing conformal field theory) we are working in the small $\lambda$ limit, the integrality of a quantity that is of order $1 / \lambda$ is not readily visible. For example, the number of wrapped Dirichlet fivebranes equals the number of $R R$ flux quanta on $\mathbf{S}^{3}$. To see integrality of this number, one should study $D$-strings on $\mathrm{AdS}_{3} \times \mathbf{S}^{3}$, rather than the elementary strings that we will in fact be studying.

By contrast, integrality of the NS flux can be probed by elementary strings. If one considers on $\mathbf{R}^{6} \times \mathrm{K} 3 \mathrm{NS}$ fivebranes as well as Dirichlet fivebranes, one gets an $\mathrm{AdS}_{3} \times \mathbf{S}^{3}$ model with NS as well as RR flux. The NS flux appears as a Wess-Zumino coupling in the $S U^{\prime}(2 \mid 2)$ sigma model, which is quantized for topological reasons. Integrality of the number of NS fivebranes is thus manifest in our formalism.

Some of the massless scalar moduli in $\mathbf{R}^{6} \times \mathrm{K} 3$ (or similarly $\mathbf{R}^{6} \times \mathbf{T}^{4}$ ) get masses when one goes to $\mathrm{AdS}_{3} \times \mathbf{S}^{3} \times \mathrm{K} 3$. In fact, one scalar in each tensor multiplet gets mass. This is a consequence of the structure of the $\mathrm{AdS}_{3} \times \mathbf{S}^{3}$ supermultiplets, and hence will automatically be true in the framework we are developing here. However, we will not describe the vertex operators for disturbances in $\mathrm{AdS}_{3} \times \mathbf{S}^{3}$ in the present paper and so will not see the phenomenon explicitly.

\section{2. $N=2$ and $N=4$ Topological Strings}

The aim of this section is to recall the definition of $N=2$ [15] and $N=4$ [4] topological strings. Then we will proceed in section 3 to review how the superstring can be formulated in the language of $N=4$ topological string theory, and in section 4 to review how this formalism can be used to give a description of the superstring with manifest $D=6$ spacetime supersymmetry. In section 5 , we will review the massless vertex operators in this spacetime-supersymmetric description of the superstring. 


\section{1. $N=2$ Topological Strings}

The $N=2$ topological string is modeled after ordinary $N=0$ bosonic strings. Thus to motivate it, we first recall what general ingredients are needed for defining a bosonic string theory.

In bosonic string perturbation theory, the key elements are a two-dimensional conformal field theory with certain additional structures. 1 One requires a spin 1 fermionic BRST current $j_{B R S T}$; the charge associated to it is the BRST operator

$$
Q_{B R S T}=\oint j_{B R S T}
$$

and obeys $Q_{B R S T}^{2}=0 . j_{B R S T}$ has charge one with respect to a "ghost number" symmetry, which is generated by a ghost current $j_{g h o s t}$ that has an anomaly $3 g-3$ on a Riemann surface of genus $g$. In addition there is a spin 2 antighost field $b$ with ghost number -1 , which satisfies

$$
\{Q, b(z)\}=T(z)
$$

where $T$ is the total energy momentum tensor of the system (including both the matter and ghost system). $T$ has central charge 0 . Moreover $(b)^{2} \sim 0$ (in the sense that there is no short distance singularity in the $b \cdot b$ operator product). The physical fields are defined relative to $Q_{B R S T}$ cohomology. A physical field is defined to be a ghost number 1 field $\Phi^{+}$ that obeys

$$
\left\{Q_{B R S T}, \Phi^{+}\right\}=0
$$

with the gauge equivalence relation being

$$
\Phi^{+} \sim \Phi^{+}+\left\{Q_{B R S T}, \Lambda\right\}
$$

For conventional bosonic strings, one can prove from the structure of the BRST cohomology that every physical field, modulo the gauge equivalence relation, has a representative that is a primary field of the Virasoro algebra of dimension 0. We will assume in general that $\Phi^{+}$has this property. Let $b_{-1} \Phi^{+}$denote the single pole in the OPE of $b$ with $\Phi^{+}$. (In what follows, in general, if $O$ is an operator of dimension $d, O_{n} \Phi$ will signify the pole of order $d+n$ in the OPE of $O$ with $\Phi$.) Then the operator $\int b_{-1} \Phi^{+}$has dimension one and so is a marginal deformation that can be used to give a deformation of the action. By

1 We will concentrate on left-movers and will denote right-movers with barred notation. 
virtue of the properties explained above, the operator $\int b_{-1} \Phi^{+}$has ghost number zero, is BRST invariant modulo a surface term, and commutes with $b$. So adding it to the action preserves the structure we have assumed for the definition of the theory, namely the conformal invariance, BRST invariance, ghost symmetry, and the existence of $b$.

Now, let us consider the theory formulated on a genus $g$ Riemann surface. The moduli space of such surfaces is a complex $3 g-3$ dimensional space $\mathcal{M}_{g}$. The tangent vectors on it are identified with Beltrami differentials $\mu_{z}^{z}$ on the corresponding Riemann surface. Beltrami differentials are equivalent if they differ by $\bar{\partial} v^{z}$ where $v^{z}$ is a globally defined vector field on the Riemann surface. Modulo this relation, the space of Beltrami differentials has dimension $3 g-3$ (for $g>1$ ). Note that since $b$ is a spin 2 current, i.e., it is a quadratic differential on the Riemann surface, the integral

$$
b(\mu)=\int b_{z z} \mu_{\bar{z}}^{z}
$$

makes sense on the Riemann surface. The $N$-point genus $g$ bosonic string scattering amplitude is defined by

$$
F_{g}=\int_{\mathcal{M}_{g}} \prod_{i}\left\langle\left|\prod_{i=1}^{3 g-3} b\left(\mu_{i}\right)\right|^{2} \prod_{r=1}^{N} \int b_{-1} \bar{b}_{-1} \Phi_{(r)}^{++}\right\rangle
$$

where the $\mu_{i}$ fix the volume element on $\mathcal{M}_{g}$ and $\Phi_{(r)}^{++}$satisfies the physicality conditions with respect to both left and right-movers. It is easy to check that this definition is consistent with the notion of physical states defined above. In particular, if we add a term $Q \Lambda$ to one of the $\Phi_{(r)}^{+}$, this does not affect the genus $g$ partition function. Also note that in order for $F_{g}$ not to be trivially zero, the ghost current needs to have an anomaly. In particular, there must be $3 g-3$ units of ghost charge generated by the path-integral measure in order to balance the $3 g-3$ anti-ghost $b$ fields in the definition of $F_{g}$.

Now we are ready to discuss $N=2$ topological strings. Start with any $N=2$ superconformal field theory. This will have currents $T, G^{ \pm}$, and $J$ of spins $2, \frac{3}{2}$, and 1 , respectively, where $T$ is the energy momentum tensor, $J$ is the $U(1)$ current, and $G^{ \pm}$are the two supercurrents with charge \pm 1 under $J$. The energy-momentum tensor can be modified or "twisted" by

$$
T \rightarrow T^{\prime}=T-\frac{1}{2} \partial J
$$

(or the same formula with $+\frac{1}{2} \partial J$ instead). One can show that $T^{\prime}$ generates a Virasoro algebra. Passing from $T$ to $T^{\prime}$ shifts the dimension of every field by $-1 / 2$ its $U(1)$ charge. 
So with respect to $T^{\prime}, G^{+}$has spin 1 and $G^{-}$has spin 2. Moreover, the modified energy momentum tensor has zero central charge no matter what was the central charge $\hat{c}$ of the underlying $N=2$ theory that we started with. Now we see we have exactly the same structure as needed in the definition of bosonic string with the following identifications:

$$
\begin{gathered}
T \rightarrow T^{\prime} \\
j_{B R S T} \rightarrow G^{+} \\
b \rightarrow G^{-} \\
j_{\text {ghost }} \rightarrow J .
\end{gathered}
$$

In particular, (2.2) now takes the form

$$
F_{g}=\int_{\mathcal{M}_{g}} \prod_{i}\left\langle\left|\prod_{i=1}^{3 g-3} G^{-}\left(\mu_{i}\right)\right|^{2} \prod_{r=1}^{N} \int G_{-1}^{-} \bar{G}_{-1}^{-} \Phi_{(r)}^{++}\right\rangle
$$

Translating the conditions for the physical fields, we see that they correspond to chiral primary fields with charge +1 and dimension 0 (they arise from operators that have charge 1 and dimension $\frac{1}{2}$ before twisting).

Note also that nonvanishing of the partition function puts a strong constraint on the anomaly of the $U(1)$ current. Unless the anomaly of the $N=2 U(1)$ current is $3 g-3$ at genus $g$, the partition function trivially vanishes and we must consider correlation functions of suitable physical states instead. If the anomaly is precisely $3 g-3$, the partition function will generally not vanish. The anomaly arises from a two point function $J \cdot T^{\prime}=$ $J \cdot\left(T-\frac{1}{2} \partial J\right)$. Since the $J \cdot T$ two point function vanishes (by virtue of the underlying $N=2$ algebra), the anomaly comes from $J \cdot \partial J$, and hence is proportional to the central charge $\hat{c}$ of the $N=2$ algebra. The anomaly is in fact given precisely by by $\hat{c}(g-1)$, so $\hat{c}=3$ is the case for which the partition function need not a priori vanish. An important example of an $N=2$ model with $\hat{c}=3$ is the supersymmetric sigma model with target space a Calabi-Yau threefold. 


\section{2. $N=4$ topological strings}

Let us consider an $N=4$ superconformal theory and see how one can make a string theory out of that, as we have just done for $N=2$ superconformal theories. By an $N=4$ theory, we mean a superconformal theory with the small $N=4$ algebra, which has one spin 2 energy momentum tensor $T$, four spin $\frac{3}{2}$ fermionic currents $G^{\alpha}$, and three spin 1 currents $J^{a}$ forming an $S U(2)$ current algebra. We write the supercurrents as $G^{a, i}$, $a, i=1,2$, obeying in a unitary theory a reality condition $G^{a, i}=\epsilon^{a b} \epsilon^{i j} G_{b, j}^{*}$. The $S U(2)$ symmetry that is part of the $N=4$ algebra acts on the first index of $G^{a, i}$. Acting on the second index is an additional $S U(2)$ symmetry, which we call $S U(2)_{\text {outer }}$, that acts by outer automorphisms on the $N=4$ algebra. Some $N=4$ theories have $S U(2)_{\text {outer }}$ as a symmetry (for instance, the sigma model with target the flat hyper-Kahler manifold $\mathbf{R}^{4}$ ) but many others do not (for instance, a sigma model with target a K3 surface).

In order to define the $N=4$ topological string, it is convenient to first view the $N=4$ superconformal theory as an $N=2$ superconformal theory. To pick an $N=2$ subalgebra in an $N=4$ theory, we proceed as follows. First we choose, from among the $S U(2)$ currents $J^{a}$, a linear combination that we will regard as the $U(1)$ current $J$ of an $N=2$ algebra. However, even when $J$ is given, an $N=2$ subalgebra of the $N=4$ algebra is not uniquely determined. With respect to $J$, there are two supercurrents of charge 1 , which we may denote as $G^{+, i}, i=1,2$. We may take any linear combination of these to be the $G^{+}$ generator of an $N=2$ algebra $\left(G^{-}\right.$will then be fixed as the hermitian conjugate of $\left.G^{+}\right)$. The first choice, namely the choice of $J$, is inessential in the sense that the different choices give equivalent theories; they differ by $S U(2)$ rotations. However, the choice of which linear combination of the $G^{+, i}$ we call $G^{+}$contains essential information. The choices can indeed be rotated to each other by $S U(2)_{\text {outer }}$, but in general, as we have noted, $S U(2)_{\text {outer }}$ is not a symmetry of the theory. So the different choices are inequivalent.

If we denote the $G^{+, i}$ as $G^{+}$and $\widetilde{G}^{+}$, then we can take the $G^{+}$generator in the $N=2$ algebra to be

$$
\widehat{G^{+}}(u)=u_{1} G^{+}+u_{2} \widetilde{G}^{+}
$$

for any complex constants $u_{1}, u_{2}$. If the $u_{i}$ are multiplied by a common factor, we get the same $N=2$ algebra, so the choices are parametrized by a copy of $\mathbf{C P}^{1}$. We can think of this $\mathbf{C P}^{1}$ as a two-sphere that is a homogeneous space of the group $S U(2)_{\text {outer }}$; it can be identified as

$$
\frac{S U(2)_{\text {outer }}}{U(1)}
$$


As we have stressed, since the $S U(2)_{\text {outer }}$ is an outer automorphism of the algebra and is not necessarily a symmetry of the theory, the different $u$ 's will give inequivalent imbeddings of the $N=2$ superconformal algebra in $N=4$. For any choice of the $u_{i}$, normalized to $\left|u_{1}\right|^{2}+\left|u_{2}\right|^{2}=1$, we pick the basis

$$
\begin{aligned}
& \widehat{G^{+}}(u)=u_{1} G^{+}+u_{2} \widetilde{G}^{+} \\
& \widehat{\widetilde{G}^{-}}(u)=-u_{2} G^{-}+u_{1} \widetilde{G}^{-} \\
& \widehat{G^{-}}(u)=u_{1}^{*} G^{-}+u_{2}^{*} \widetilde{G}^{-} \\
& \widehat{\widetilde{G}^{+}}(u)=-u_{2}^{*} G^{+}+u_{1}^{*} \widetilde{G}^{+} .
\end{aligned}
$$

These formulas have been obtained as follows. We have regarded $\left(u_{1}, u_{2}\right)$ as the top row of an $S U(2)_{\text {outer }}$ matrix

$$
M=\left(\begin{array}{cc}
u_{1} & u_{2} \\
-u_{2}^{*} & u_{1}^{*}
\end{array}\right) .
$$

Then we have transformed the two $S U(2)_{\text {outer }}$ doublets, namely $\left(G^{+}, \widetilde{G}^{-}\right)$and $\left(\widetilde{G}^{+}, G^{-}\right)$, by this group element to get the above basis.

Either $\left(G^{+}, G^{-}\right)$or $\left(\widetilde{G}^{+}, \widetilde{G}^{-}\right)$, or their transforms by $M$, can be used to form the two supercurrents of an $N=2$ algebra. Moreover, the OPEs

$$
G^{+} \widetilde{G}^{-} \sim 0 \quad G^{-} \widetilde{G}^{+} \sim 0
$$

have no singularities.

We now construct an $N=2$ twisted theory by modifying the energy momentum tensor as explained before: $T \rightarrow T-\frac{1}{2} \partial J$. Since this operation shifts the dimension of every field by $-\frac{1}{2}$ its $U(1)$ charge, the twisted theory has the following symmetry currents. There are two bosonic spin 2 fields, namely the modified energy momentum tensor $T^{\prime}$ and the $S U(2)$ current $J^{--}$, and two fermionic spin 2 currents $G^{-}, \widetilde{G}^{-}$. Moreover, there are two spin 1 fermionic currents $G^{+}, \widetilde{G}^{+}$and a bosonic spin one current $J$. In addition, there is a spin 0 bosonic current of $S U(2)$, namely $J^{++}$.

Now we have to decide which fields in the twisted theory we would like to identify as "physical." In the case of $N=2$ topological theories, we identified the physical spectrum with the cohomology of $G^{+}$, i.e. with the space of fields annihilated by $G^{+}$modulo addition of fields of the form $G^{+} \Lambda$. Now we have in principle two BRST charges, because both $G^{+}$ 
and $\widetilde{G}^{+}$are spin 1 fermionic currents and give rise to nilpotent fermionic charges $G_{0}^{+}$and $\widetilde{G}_{0}^{+}$. Moreover we have

$$
\left\{G_{0}^{+}, \widetilde{G}_{0}^{+}\right\}=0
$$

by virtue of the $N=4$ algebra. It is natural to construct a theory in which a physical field is required to be annihilated by both $G_{0}^{+}$and $\widetilde{G}_{0}^{+}$. Any field of the form $\left[G_{0}^{+},\left[\widetilde{G}_{0}^{+}, \Lambda\right]\right]$ (where [, ] denotes the appropriate commutator or anticommutator) obeys this condition; we consider such fields to be trivial. In other words, we identify the physical fields as Virasoro primary fields $\Phi^{+}$satisfying

$$
\left[G_{0}^{+}, \Phi^{+}\right]=\left[\widetilde{G}_{0}^{+}, \Phi^{+}\right]=0, \quad \Phi^{+} \sim \Phi^{+}+\left[G_{0}^{+},\left[\widetilde{G}_{0}^{+}, \Lambda^{-}\right]\right]
$$

As before, to the physical field $\Phi^{+}$, we associate the deformation of the Lagrangian $\int G_{-1}^{-} \Phi^{+}$.

One would like to know under what conditions this deformation preserves the $N=4$ structure. A simple sufficient criterion, which will be convenient when we discuss massless vertex operators, is that $\int G_{-1}^{-} \Phi^{+}$should preserve the $S U(2)$ symmetry. (This condition suffices since $N=2$ plus $S U(2)$ gives $N=4$.) In particular, acting with the raising operator $J^{++}$(and recalling that, after twisting, its zero mode becomes $J_{1}^{++}$), we get $J_{1}^{++} G_{-1}^{-} \Phi^{+}=G_{-1}^{-} J_{1}^{++} \Phi^{+}+\widetilde{G}_{0}^{+} \Phi^{+}=G_{-1}^{-} J_{1}^{++} \Phi^{+}$. A sufficient criterion for vanishing of $J_{1}^{++} G_{-1}^{-} \Phi^{+}$is thus $J_{1}^{++} \Phi^{+}=0$. In terms of the untwisted theory, this means that $\Phi^{+}$, having $U(1)$ charge $1 / 2$ and being annihilated by $J^{++}$, is the top component of an $S U(2)$ doublet. As explained in section 2 of reference [4, the condition that the zero mode of $J^{--}$annihilates the deformation is that $G^{-}\left(J_{-1}^{--} \Phi^{+}\right)=0$, in other words the bottom component of the doublet containing $\Phi^{+}$is antichiral from the $N=2$ point of view.

Suppose the cohomology of $\widetilde{G}_{0}^{+}$is trivial, i.e. $\widetilde{G}_{0}^{+} V=0$ implies $V=\widetilde{G}_{0}^{+} W$ for some $W$. Then if we were able to consider a reduced Hilbert space $\widetilde{\mathcal{H}} \subset \mathcal{H}$ where $\widetilde{\mathcal{H}}$ is the subspace of $\mathcal{H}$ killed by $\widetilde{G}_{0}^{+}$, the physical state condition (2.6) acting on this reduced Hilbert space would be the same as the condition for an $N=2$ twisted physical field 2 and we could use the same rules of computation as discussed in the previous subsection. So the question is how to do this reduction. Using the fact that $\left(\widetilde{G}^{+}\right)^{2}=0$, this can be done by simply

2 In other words, consider the $G_{0}^{+}$cohomology in $\widetilde{\mathcal{H}}$. It is generated by operators $\Phi \in \widetilde{\mathcal{H}}$ obeying $\left[G_{0}^{+}, \Phi\right]=0$; such states, being in $\widetilde{\mathcal{H}}$, obey also $\widetilde{G}_{0}^{+} \Phi=0$. $\Phi$ is considered trivial if it is of the form $G_{0}^{+} \Psi$ for some $\Psi \in \widetilde{\mathcal{H}}$; if the $\widetilde{G}^{+}$cohomology is trivial, we can write $\Psi=\left[\widetilde{G}_{0}^{+}, \Lambda\right]$, and then the equivalence relation on $\Phi$ is $\Phi \sim \Phi+\left[G_{0}^{+},\left[\widetilde{G}_{0}^{+}, \Lambda\right]\right]$. 
inserting an operator $\oint \widetilde{G}^{+}$around each $a$-cycle on the Riemann surface, or in a manifestly modular-invariant way by combining with right-movers and integrating $\int d^{2} z \widetilde{G}^{+} \widetilde{\widetilde{G}}^{+}$over the surface (when $\widetilde{G}^{+}$is holomorphic, this surface integral reduces to integrals over the cycles in the usual way). We are therefore led to considering the $N$-point scattering amplitude on genus $g$ defined by the measure over moduli space $\mathcal{M}_{g}$

$$
\left\langle\left|G^{-}\left(\mu_{1}\right) \ldots G^{-}\left(\mu_{3 g-3}\right)\right|^{2}\left[\int \widetilde{G}^{+} \widetilde{\bar{G}}^{+}\right]^{g} \prod_{r=1}^{N} \int G_{-1}^{-} \bar{G}_{-1}^{-} \Phi_{(r)}^{++}\right\rangle
$$

However, this guess requires some modification, because this amplitude is identically zero. To see this, use $\oint \widetilde{G}^{+} J=-\widetilde{G}^{+}$to replace one of the $\widetilde{G}^{+}$'s by the contour integral of $\oint \widetilde{G}^{+}$around $J$, and pull the contour of $\widetilde{G}^{+}$off the surface. Since the $G^{-}$'s and $\widetilde{G}^{+}$'s have no singularity with it, we get zero! There is another reason why the above formula is not what we want. From (2.6) we wish a deformation to be trivial only if it can be written as $\left[G_{0}^{+},\left[\widetilde{G}_{0}^{+}, \Lambda\right]\right]$. However, in the above definition, it is easy to see that $\left[\widetilde{G}_{0}^{+}, \Lambda\right]$ would already be topologically trivial since we can pull the $\oint \widetilde{G}^{+}$contour off of $\Lambda$ and get zero by the same reasoning as above.

So instead, we will define the topological $N$-point scattering amplitude to be

$$
F_{g}=\int_{\mathcal{M}_{g}}\left\langle\left|G^{-}\left(\mu_{1}\right) \ldots G^{-}\left(\mu_{3 g-3}\right)\right|^{2}\left[\int \widetilde{G}^{+}{\widetilde{G^{+}}}^{g-1} \int J \bar{J} \prod_{r=1}^{N} \int G_{-1}^{-} \bar{G}_{-1}^{-} \Phi_{(r)}^{++}\right\rangle\right.
$$

which is no longer zero. Note that the contour of $\oint \widetilde{G}^{+}$can no longer be pulled off the surface since it hits the $J$ and gives back $\widetilde{G}^{+}$as the residue. For the same reason, adding $\oint \widetilde{G}^{+} \Lambda$ to the action may change the partition function. However, if we consider adding $\oint G^{+} \oint \widetilde{G}^{+} \Lambda$ to the action, then the $\widetilde{G}^{+}$contour can be pulled off of $\Lambda$ and converts the $J$ to a $\widetilde{G}^{+}$. Now pulling the $G^{+}$contour off of $\Lambda$, we encounter no residues from $\widetilde{G}^{+}$. From the $G^{-}$'s, we get residues which are the energy momentum tensor, thus giving us total derivatives in the moduli which at least formally (barring anomalies) integrate to zero. Thus (2.7) has the correct topological symmetry.

Actually, because of the possibility of making an $S U(2)_{\text {outer }}$ rotation of the $N=2$ embedding, there is a whole family of topological scattering amplitudes which can be defined by

$$
\hat{F}_{g}\left(u_{1}^{*}, u_{2}^{*}, \bar{u}_{1}^{*}, \bar{u}_{2}^{*}\right)=\int_{\mathcal{M}_{g}}\left\langle\left|\widehat{G}^{-}\left(\mu_{1}\right) \ldots \widehat{G}^{-}\left(\mu_{3 g-3}\right)\right|^{2}\left[\int \widehat{\widetilde{G}}^{+} \widehat{\widetilde{G}}^{+}\right]^{g-1} \int J \bar{J}\right.
$$




$$
\left.\prod_{r=1}^{N} \int \widehat{G}_{-1}^{-} \widehat{\widehat{G}}_{-1}^{-} \phi_{(r)}^{++}\right\rangle
$$

where $\widehat{\widetilde{G}}^{+}$and $\widehat{G}^{-}$are defined in (2.4). (Note that $\bar{u}_{1}$ and $\bar{u}_{2}$ refer to the right-movers, and are not the complex conjugates of $u_{1}$ and $u_{2}$, which are denoted as $u_{1}^{*}$ and $u_{2}^{*}$.) Since $\hat{F}_{g}$ is a homogeneous polynomial of degree $4 g-4+N$ in $\left(u_{1}^{*}, u_{2}^{*}\right)$ and degree $4 g-4+N$ in $\left(\bar{u}_{1}^{*}, \bar{u}_{2}^{*}\right)$, we can decompose it to each individual component of the polynomial and obtain $(4 g-3+N)^{2}$ independent scattering amplitudes $F_{g}^{(m, n)}$ defined by

$$
\begin{gathered}
F_{g}\left(u_{1}^{*}, u_{2}^{*}, \bar{u}_{1}^{*}, \bar{u}_{2}^{*}\right)= \\
\sum_{m=-2 g+2-N}^{2 g-2} \sum_{n=-2 g+2-N}^{2 g-2} \frac{(4 g-4+N) !}{(2 g-2+N+m) !(2 g-2-m) !} \frac{(4 g-4+N) !}{(2 g-2+N+n) !(2 g-2-n) !} \\
F_{g}^{(m, n)}\left(u_{1}^{*}\right)^{2 g-2+N+m}\left(u_{2}^{*}\right)^{2 g-2-m}\left(\bar{u}_{1}^{*}\right)^{2 g-2+N+n}\left(\bar{u}_{2}^{*}\right)^{2 g-2-n} .
\end{gathered}
$$

Comparing with (2.8), one sees that $F_{g}^{(m, n)}$ is computed in the same manner as $F_{g}^{(2 g-2,2 g-2)}$ of (2.7) but with some of the $G^{-}$'s and $\widetilde{G}^{+}$'s switched to $\widetilde{G}^{-}$'s and $G^{+}$'s. Using contour integral manipulations similar to those described above, it is straightforward to show that switching any $p G^{-}$'s to $\widetilde{G}^{-}$'s and any $q \widetilde{G}^{+}$'s to $G^{+}$'s in (2.7) computes $F^{(2 g-2-p-q, 2 g-2)}$ up to contact terms. The choice of which $G^{-}$'s and $\widetilde{G}^{+}$'s are switched is irrelevant up to contact terms.

For example, consider the amplitude below where one of the $G^{-}$'s in the measure has been switched to a $\widetilde{G}^{-}$:

$$
\begin{gathered}
F_{g}=\int_{\mathcal{M}_{g}}\left\langle\widetilde{G}^{-}\left(\mu_{1}\right) \bar{G}^{-}\left(\bar{\mu}_{1}\right)\left|G^{-}\left(\mu_{2}\right) \bar{G}^{-}\left(\bar{\mu}_{2}\right) \ldots G^{-}\left(\mu_{3 g-3}\right)\right|^{2}\left[\int \widetilde{G}^{+} \overline{\widetilde{G}}^{+}\right]^{g-1} \int J \bar{J}\right. \\
\left.\prod_{r=1}^{N} \int G_{-1}^{-} \bar{G}_{-1}^{-} \phi_{(r)}^{++}\right\rangle .
\end{gathered}
$$

Writing $\widetilde{G}^{-}\left(\mu_{1}\right)=\left[\oint G^{+}, J^{--}\left(\mu_{1}\right)\right]$, one can pull the $G^{+}$contour off the $J^{--}\left(\mu_{1}\right)$ until it hits the $J$ to turn it into a $G^{+}$. (We are ignoring contact terms coming from when the $G^{+}$ hits the $G^{-}$'s.) One can now write one of the $\widetilde{G}^{+}$'s from the cycles as $\widetilde{G}^{+}=\left[\oint \widetilde{G}^{+}, J\right]$, and pull the $\widetilde{G}^{+}$contour off until it hits the bare $J^{--}\left(\mu_{1}\right)$, turning it into a $G^{-}\left(\mu_{1}\right)$. Finally, one pulls the $G^{+}$off one of the cycles until it hits the $J$ on the other cycle to give the amplitude

$$
F_{g}=\int_{\mathcal{M}_{g}}\left\langle\left|G^{-}\left(\mu_{1}\right) \ldots G^{-}\left(\mu_{3 g-3}\right)\right|^{2}\left[\int \widetilde{G}^{+} \overline{\widetilde{G}}^{+}\right]^{g-2} \int G^{+} \overline{\widetilde{G}}^{+} \int J \bar{J}\right.
$$




$$
\left.\prod_{r=1}^{N} \int G_{-1}^{-} \bar{G}_{-1}^{-} \phi_{(r)}^{++}\right\rangle .
$$

So we have shown that the amplitude is the same (up to contact terms) if one switches a $G^{-}$to $\widetilde{G}^{-}$or if one switches a $\widetilde{G}^{+}$to $G^{+}$.

\subsection{Critical $N=2$ String as an $N=4$ Topological String}

One class of $N=4$ theories that is important for our purposes is the following. One can construct a small $N=4$ superconformal theory from any $\hat{c}=2 N=2$ superconformal theory by defining the three $\mathrm{SU}(2)$ currents to be $J, e^{\int J}$ and $e^{-\int J}$, and defining the four

fermionic generators to be $G^{+}, G^{-},\left[e^{-\int J}, G^{+}\right]$and $\left[e^{\int J}, G^{-}\right]$. Note that $\hat{c}$ must equal 2 (i.e. the associated $N=2$ string is critical) in order that $e^{ \pm \int J}$ has spin 1 . 3

It is interesting to ask what $F_{g}^{(m, n)}$ is computing for these $N=4$ strings. As was shown in reference [4], $F_{g}^{(m, n)}$ computes the $N=2$ scattering amplitude on a surface of leftmoving instanton number $m$ and right-moving instanton number $n$. In other words, after introducing the usual set of $N=2$ super-reparameterization ghosts, with $c=-6$, one can construct BRST-invariant vertex operators for the critical $N=2$ string which are in oneto-one correspondence with the physical vertex operators of the $N=4$ topological string. Furthermore, one can compute $N=2$ correlation functions of these vertex operators on an $N=2$ super-Riemann surface of genus $g$ and left and right-moving instanton number $m$ and $n$, and after integrating over the $N=2$ super-moduli of this Riemann surface (including the fermionic and $U(1)$ moduli), one recovers precisely the $N=4$ topological formula $F_{g}^{(m, n)}$ for the appropriate $N=4$ physical vertex operators.

\section{The Superstring as an $N=4$ Topological String}

We will now review how the usual superstring (which is conventionally described as a critical $N=1$ string) can also be described as an $N=2$ string with $\hat{c}=2$, and therefore also as an $N=4$ topological string. The advantage of the $N=4$ topological description

3 An important example of this construction is the following. In general, the supersymmetric sigma model with target a Calabi-Yau $n$-fold is an $N=2$ superconformal model with $\hat{c}=n$. To get $N=4$ supersymmetry, the target should be hyper-Kahler. The condition $\hat{c}=2$ amounts to $n=2$. A Calabi-Yau two-fold is always hyper-Kahler, and that is why the $\hat{c}=2$ case automotically gives $N=4$ superconformal symmetry. 
of the superstring is that, after a field-redefinition to Green-Schwarz-like variables, some of the spacetime supersymmetry can be made manifest. In this paper, we will be mainly interested in Type IIB superstring compactifications on K3, which admit sixteen unbroken spacetime supersymmetries. (We can also consider compactification on $\mathbf{T}^{4}$, but in that case our construction exhibits only some of the unbroken supersymmetries.) As will be reviewed below, eight of these sixteen supersymmetries will be manifest in the formalism (i.e. act geometrically on the target superspace), while the other eight can be exhibited in terms of ordinary vertex operators (as opposed to spin operators) but do not arise just from geometrical symmetries of the target superspace.

We shall first construct the $N=4$ superconformal generators for the RNS superstring and prove that the physical $N=4$ vertex operators are in one-to-one correspondence with the usual BRST-invariant RNS vertex operators. We shall then show that the $N=4$ topological amplitude $F_{g}^{(m, n)}$ defined in the previous section computes the RNS $g$-loop superstring amplitude where the total left and right-moving picture of the vertex operators is $m$ and $n$.

\section{1. $N=4$ Superconformal Generators and Physical Vertex Operators}

As discussed in [16], any critical $N=1$ string can be embedded into a critical $N=2$ string. This embedding allows $N=1$ string scattering amplitudes to be computed using $N=2$ string methods.

The critical $N=2$ superconformal generators are constructed from the $N=1$ matter and ghost fields as follows:

$$
\begin{gathered}
T=T_{N=1}+\frac{1}{2} \partial(b c+\xi \eta) \\
G^{+}=\gamma G_{m}+c\left(T_{m}-\frac{3}{2} \beta \partial \gamma-\frac{1}{2} \gamma \partial \beta-b \partial c\right)-\gamma^{2} b+\partial^{2} c+\partial(c \xi \eta) \\
G^{-}=b \\
J=c b+\eta \xi
\end{gathered}
$$

where $T_{N=1}=T_{m}+T_{g}$ is the stress-tensor of the original $N=1$ matter and ghost fields, and the $(\beta, \gamma)$ super-reparameterization ghosts have been bosonized as $\left(\beta=i e^{-\phi} \partial \xi, \gamma=\right.$ $\left.-i \eta e^{\phi}\right)$. 1 For instance, the zero mode of $G^{+}$is the $N=1$ BRST operator $Q$ and after

4 The factors of $i$ have been put in the bosonization formula so that $\beta$ is real, i.e. $\beta^{*}=$ $-i \partial \xi e^{-\phi}=i e^{-\phi} \partial \xi=\beta$. 
twisting (which in this case amounts to removing the term $\frac{1}{2} \partial(b c+\xi \eta)$ from $\left.T\right), G^{+}$has conformal weight 1 while $G^{-}$has conformal weight 2 . Furthermore, $J$ is related to the usual ghost current $j_{\text {ghost }}=c b+\gamma \beta$ by

$$
J=j_{\text {ghost }}-j_{\text {picture }}
$$

where $j_{\text {picture }}$ is the picture-current defined as $j_{\text {picture }}=-\partial \phi+\xi \eta$. Note that $P=\oint j_{\text {picture }}$ commutes with the $\beta$ and $\gamma$ ghosts, but not with $\eta$ or $\xi$. Moreover, we can write

$$
\oint J=Q_{g h o s t}-P
$$

where $Q_{\text {ghost }}=\oint j_{\text {ghost }}$ is the ghost number.

Since one now has a critical $N=2$ string, one can ask what does the $N=4$ topological scattering amplitude defined in the previous section compute? To answer this, first note that the additional $N=4$ generators (constructed as in subsection 2.3) are given by

$$
\begin{gathered}
\widetilde{G}^{+}=\eta \\
\widetilde{G}^{-}=b\left(e^{\phi} G_{m}+\eta e^{2 \phi} \partial b-c \partial \xi\right)-\xi\left(T_{m}-\frac{3}{2} \beta \partial \gamma-\frac{1}{2} \gamma \partial \beta+2 b \partial c-c \partial b\right)+\partial^{2} \xi, \\
J^{++}=c \eta, \quad J^{--}=b \xi .
\end{gathered}
$$

So physical $N=4$ topological vertex operators $\Phi^{+}$are defined by the conditions $(2.6)$, i.e.

$$
Q \Phi^{+}=\eta_{0} \Phi^{+}=\left(Q_{\text {ghost }}-P-1\right) \Phi^{+}=0, \quad \Phi^{+} \sim \Phi^{+}+Q \eta_{0} \Lambda^{-}
$$

But these are just the physical conditions for a BRST-invariant vertex operator in the standard RNS formalism. Note that the condition $\eta_{0} \Phi^{+}=0$ implies that $\Phi$ is independent of the $\xi$ zero mode (i.e. it can be written in terms of the unfermionized $\beta$ and $\gamma$ ghosts) and, similarly, the triviality condition $\Phi^{+}=Q \eta_{0} \Lambda^{-}$implies that $\Phi^{+}=Q \Omega$ for some $\Omega$ which is independent of the $\xi$ zero mode (i.e. $\eta_{0} \Omega=0$ ). Furthermore, $(G-P-1) \Phi^{+}=0$ is the usual ghost-number condition for physical fields.

5 For NS states at zero picture, it implies that $G=1$ as desired. For $\mathrm{R}$ states at picture $-1 / 2$, it implies that $G=1 / 2$ as desired. All RNS physical states at other pictures are related to these pictures by multiplication by the picture-raising operator $Z=\{Q, \xi\}$ or the picture-lowering operator $Y=c \partial \xi e^{-2 \phi}$. But the operators $Z$ and $Y$ commute with $\oint J$, so these other physical states also satisfy the condition $(\oint J-1) \Phi^{+}=0$. 


\section{2. $N=4$ Topological Amplitudes as RNS Superstring Amplitudes}

In terms of the RNS variables, the scattering amplitude defined in (2.7) (or in (2.8) at $\left.u_{2}=\bar{u}_{2}=0\right)$ is

$$
\begin{gathered}
F_{g}^{(2 g-2,2 g-2)}=\int_{\mathcal{M}_{g}}\left\langle\left|b\left(\mu_{1}\right) \ldots b\left(\mu_{3 g-3}\right)\right|^{2}\left[\int \eta \bar{\eta}\right]^{g-1} \int(b c+\xi \eta)(\bar{b} \bar{c}+\bar{\xi} \bar{\eta})\right. \\
\left.\prod_{r=1}^{N} \int b_{-1} \bar{b}_{-1} \Phi_{(r)}^{++}\right\rangle .
\end{gathered}
$$

Note that if we are in the "large" RNS Hilbert space, i.e. including the $\xi$ zero mode, then the only $\xi$ zero mode in this expression comes from the $J$ current $b c+\xi \eta$. After integrating out the $\xi$ zero mode, one is left with the integration

$$
\begin{gathered}
{\left[\int d^{2} z(\eta \bar{\eta})\right]^{g}=\left[\sum_{i=1}^{g}\left(\int_{a_{i}} d z \eta \int_{b_{i}} d \overline{z \eta}-\int_{b_{i}} d z \eta \int_{a_{i}} d \overline{z \eta}\right)\right]^{g}} \\
=g ! \prod_{i=1}^{g}\left(\int_{a_{i}} d z \eta \int_{b_{i}} d \overline{z \eta}-\int_{b_{i}} d z \eta \int_{a_{i}} d \overline{z \eta}\right) .
\end{gathered}
$$

Although these $\eta$ integrations over the cycles look strange, they can be understood as coming from the integration over the $(\beta, \gamma)$ ghosts in the bosonized form of the standard RNS model [17 6. The rest of (3.4) is just the standard RNS scattering amplitude when the total (left,right)-moving picture of the vertex operators $\Phi_{(r)}^{++}$is $(2 g-2,2 g-2)$.

To obtain the scattering amplitude when the vertex operators are in other pictures, note that switching $G^{-}$with $\widetilde{G}^{-}$in the definition of the integrated vertex operator raises its picture by +1 , i.e.

$$
\int \widetilde{G}_{-1}^{-} \Phi^{+}=\int\left[G^{+}, J_{-1}^{--}\right] \Phi^{+}=\int\left[Q,(\xi b)_{-1}\right] \Phi^{+}=\int\left[Q, \xi_{0} b_{-1}\right] \Phi^{+}=\int b_{-1} Z \Phi^{+}
$$

6 As discussed in [17], one needs to also insert $\delta$-functions in the momentum of the $\phi$ field to restrict the picture of states propagating in the internal loops. These $\delta$-functions give rise to unphysical poles coming from the multiloop correlation function for the negative-energy field $\phi$ which, at the present time, are not well understood. So our scattering amplitudes are only trustworthy when these $\delta$-functions of $\partial \phi$ do not contribute, i.e. when the integrands of the scattering amplitudes are independent of the locations of the picture-changing operators and there are no unphysical poles. Besides tree and one-loop amplitudes, examples of such multi-loop scattering amplitudes are the $D=4$ and $D=6$ "topological" scattering amplitudes discussed in [18] and [4]. 
where $Z=\left\{Q, \xi_{0}\right\}$ is the picture-raising operator and we are assuming that $(\xi b)_{-1} \Phi^{+}=$ $\xi_{0} b_{-1} \Phi^{+}$, i.e. $\xi$ has no poles with $\Phi^{+}$and $b$ has no double poles or higher with $\Phi^{+}$. It will now be shown that computing $F^{(2 g-2,2 g-2)}$ in (2.8) with one of the integrated vertex operators replaced by $\int \widetilde{G}_{-1}^{-} \bar{G}_{-1}^{-} \Phi^{+}$is equivalent to computing $F^{(2 g-3,2 g-2)}$ in (2.8) where all of the vertex operators are of the form $\int G_{-1}^{-} \bar{G}_{-1}^{-} \Phi^{+}$. So if $F^{(2 g-2,2 g-2)}$ computes the RNS superstring amplitude when the total (left,right)-moving picture of the vertex operators is $(2 g-2,2 g-2)$, then $F^{(2 g-3,2 g-2)}$ computes the RNS superstring amplitude when the total (left,right)-moving picture of the vertex operators is $(2 g-3,2 g-$ 2 ). Similarly, one can show that $F^{(m, n)}$ computes the scattering amplitude when the total picture of the vertex operators is $(m, n)$.

To prove the above claim (which is a special case of the claim for $F^{(m, n)}$ made above (2.10)), consider the amplitude

$$
\begin{gathered}
F_{g}=\int_{\mathcal{M}_{g}}\left\langle\left|G^{-}\left(\mu_{1}\right) \ldots G^{-}\left(\mu_{3 g-3}\right)\right|^{2}\left[\int \widetilde{G}^{+} \overline{\widetilde{G}}^{+}\right]^{g-1} \int J \bar{J}\right. \\
\left.\int \widetilde{G}_{-1}^{-} \bar{G}_{-1}^{-} \Phi_{(1)}^{++} \prod_{r=2}^{N} \int G_{-1}^{-} \bar{G}_{-1}^{-} \Phi_{(r)}^{++}\right\rangle .
\end{gathered}
$$

Writing $\widetilde{G}^{-}=\left[\oint G^{+}, J^{--}\right]$in the vertex operator $\int \widetilde{G}_{-1}^{-} \bar{G}_{-1}^{-} \Phi^{+}$, one can pull the $G^{+}$ contour off the $J^{--}$until it hits the $J$ to turn it into a $G^{+}$. (We are ignoring contact terms coming from when the $G^{+}$hits the $G^{-}$'s.) One can now write one of the $\widetilde{G}^{+}$'s from the cycles as $\widetilde{G}^{+}=\left[\oint \widetilde{G}^{+}, J\right]$, and pull the $\widetilde{G}^{+}$contour off until it hits the bare $J^{--}$on the vertex operator, turning it into a $G^{-}$. Finally, one pulls the $G^{+}$off one of the cycles until it hits the $J$ on the other cycle to give the amplitude

$$
\begin{gathered}
F_{g}=\int_{\mathcal{M}_{g}}\left\langle\left|G^{-}\left(\mu_{1}\right) \ldots G^{-}\left(\mu_{3 g-3}\right)\right|^{2}\left[\int \widetilde{G}^{+} \overline{\widetilde{G}}^{+}\right]^{g-2} \int G^{+} \overline{\widetilde{G}}^{+} \int J \bar{J}\right. \\
\left.\prod_{r=1}^{N} \int G_{-1}^{-} \bar{G}_{-1}^{-} \Phi_{(r)}^{++}\right\rangle .
\end{gathered}
$$

But up to contact terms, this is what $F^{(2 g-3,2 g-2)}$ computes. So we have shown (up to contact terms) that the amplitude computed by $F^{2 g-3,2 g-2}$ is equal to the usual superstring amplitude when the sum of the pictures of the vertex operators is $(2 g-3,2 g-2)$. 


\section{Manifest Six-Dimensional Spacetime-Supersymmetry}

To compute superstring scattering amplitudes keeping spacetime supersymmetry manifest, it is convenient to perform a field-redefinition from the RNS variables to GreenSchwarz-like variables. At the present time, it is not possible to perform a field-redefinition which preserves ten-dimensional super-Poincaré covariance; however, it is possible to preserve either four-dimensional [3], six-dimensional [4], or $U(5)$ [5] super-Poincaré invariance. The four-dimensional formalism is useful for describing compactifications of the superstring on a Calabi-Yau three-fold; a review can be found in [3]. Since we will be interested in this paper in compactifications of the superstring on a Calabi-Yau two-fold, we will use the six-dimensional formalism [0] which will be reviewed in the following two sections.

After reviewing the six-dimensional supersymmetry algebra, we shall introduce a fieldredefinition which allows the supersymmetry to be made manifest. For compactifications which preserve six-dimensional supersymmetry, this field redefinition maps the RNS matter and ghost variables into a set of six-dimensional superspace variables plus an $N=2$ $\hat{c}=2$ superconformal field theory describing the compactification. Under the field redefinition, the $N=2 \hat{c}=2$ superconformal generators of (3.1) get mapped into a sum of $N=2 \hat{c}=0$ superconformal generators constructed from the six-dimensional superspace variables and $N=2 \hat{c}=2$ superconformal generators constructed from the compactification variables. Also, the six-dimensional supersymmetry generators get mapped under this field-redefinition into simple compactification-independent operators.

\subsection{Review of six-dimensional supersymmetry}

Our six-dimensional notation will use the fact that the rotation group $S O(6)$ is locally isomorphic to $S U(4)$; likewise, with Lorentz signature, the Lorentz group $S O(1,5)$ is locally a real form of $S U(4)$. Under the identification of the rotation group with $S U(4)$, spinors of $S O(6)$ transform as 4's or $\overline{4}$ 's of SU(4). Spinors which transform as 4's will be denoted with a raised index, $\xi^{a}$ for $a=1$ to 4 , and spinors which transform as $\overline{4}$ 's will be denoted with a lowered index $\xi_{a}$. In this notation, vectors $x^{m}$ for $m=0$ to 5 can be denoted as anti-symmetric bispinors $x^{a b}=\left(\sigma_{m}\right)^{a b} x^{m}$ where $\left(\sigma_{m}\right)^{a b}$ and $\left(\sigma_{m}\right)_{a b}$ are the $\mathrm{SO}(5,1)$ Pauli matrices, which satisfy the algebra

$$
\left(\sigma_{m}\right)^{a b}\left(\sigma_{n}\right)_{a c}+\left(\sigma_{n}\right)^{a b}\left(\sigma_{m}\right)_{a c}=2 \eta_{m n} .
$$

Also, $\left(\sigma_{m}\right)_{a b}$ is defined to be $\left(\sigma_{m}\right)_{a b}=\frac{1}{2} \epsilon_{a b c d}\left(\sigma_{m}\right)^{c d}$. 
In $N=1$ supersymmetry in six dimensions, the supersymmetry generators consist of a pair of $\overline{4}$ 's, say $q_{a}^{\alpha}$ where $\alpha= \pm$. (CPT invariance together with the pseudoreal nature of the $\overline{\mathbf{4}}$ representation of $S O(5,1)$ makes it impossible to have a supersymmetric theory with supersymmetries transforming as a single copy of the $\overline{\mathbf{4}}$.) To exhibit manifest supersymmetry, one would like a Green-Schwarz style description in terms of strings moving in a superspace with coordinates $x^{m}$ and $\theta_{a}^{\alpha}$. It is not known how to accomplish this much; what is known is only how to introduce a single multiplet $\theta_{a}$ of string coordinates, so that half of the supersymmetries act geometrically. This is reminiscent of harmonic superspace, but the worldsheet analog of the harmonic variables is not yet understood. The other half of the spacetime supersymmetries is realized by vertex operators that we will describe below. All statements in this paragraph apply to open strings or to left or right-movers of a closed string. If supersymmetric left-movers and right-movers are combined to make a Type II string, then the amount of supersymmetry is doubled - both manifest and nonmanifest. Then there are separate $q_{a}^{\alpha}$ 's carried by both left and right-movers, and separate left and right-moving $\theta$ 's.

The $N=1 D=6$ supersymmetry algebra is

$$
\left\{q_{a}^{\alpha}, q_{b}^{\beta}\right\}=\frac{1}{2} \epsilon^{\alpha \beta} \epsilon_{a b c d} P^{c d}
$$

where $P_{m}$ is the translation generator. It is useful to define $S U(2)$ generators, $R^{\alpha \beta}$, which rotate these susy generators as

$$
\left[R^{\alpha \beta}, q_{a}^{\gamma}\right]=\frac{1}{2}\left(\epsilon^{\beta \gamma} q_{a}^{\alpha}+\epsilon^{\alpha \gamma} q_{a}^{\beta}\right)
$$

This $S U(2)$ group acts by outer automorphisms of the $N=1$ supersymmetry algebra.

In the RNS formalism, the spacetime-supersymmetry generators can appear in arbitrary semi-integer picture. To construct these generators, one first needs to construct the six-dimensional spinor fields, $S_{a}$ and $S^{a}$, out of the six RNS $\psi^{m}$ fields. One writes

$$
S^{a}=e^{\frac{1}{2} \int^{z}\left( \pm \psi^{0} \psi^{1} \pm \psi^{2} \psi^{3} \pm \psi^{4} \psi^{5}\right)}
$$

with an even number of + signs. $S_{a}$ is defined by the same formula but with an odd number of + signs. The simplest picture for the susy generators is the $-1 / 2$ picture where

$$
q_{a}^{ \pm}=\oint e^{-\frac{1}{2} \phi} S_{a} e^{ \pm \frac{i}{2} H_{C}^{R N S}}
$$


and $J_{C}^{R N S}=i \partial H_{C}^{R N S}$ is the $U(1)$ current of the $N=2 \hat{c}=2$ superconformal field theory representing the compactification manifold.

However, as is well known, the susy generators defined in this picture do not satisfy off-shell the algebra of (4.1). Rather, they anti-commute to give

$$
\left\{q_{a}^{\alpha}, q_{b}^{\beta}\right\}=\epsilon^{\alpha \beta} \oint e^{-\phi} \psi_{a b}
$$

$\oint e^{-\phi} \psi_{m}$ is related to the translation generator $-i \oint \partial x_{m}$ by multiplication with the picture-raising operator ( since $Z e^{-\phi} \psi^{m}=-i \partial x^{m}$ ), but picture-changing is only a valid operation when all states are on-shell. For this reason, it is convenient to define $q_{a}^{-}$in the $-\frac{1}{2}$ picture but to define $q_{a}^{+}$in the $+\frac{1}{2}$ picture. This gives

$$
\begin{gathered}
q_{a}^{-}=\oint e^{-\frac{1}{2} \phi} S_{a} e^{-\frac{i}{2} H_{C}^{R N S}} \\
q_{a}^{+}=\oint\left(e^{\frac{3}{2} \phi} b \eta S_{a} e^{\frac{i}{2} H_{C}^{R N S}}-e^{\frac{1}{2} \phi}\left(\frac{i}{2} \epsilon_{a b c d} S^{b} \partial x^{c d}+i S_{a} G_{-3 / 2 C}^{-R N S}\right) e^{\frac{i}{2} H_{C}^{R N S}}\right) .
\end{gathered}
$$

As discussed in [1], $q_{a}^{+}$is computed by multiplying the susy generator in the $-\frac{1}{2}$ picture by the picture-raising operator $Z$. The terms in $q_{a}^{+}$come from the terms $\partial \phi b \eta e^{2 \phi},-i e^{\phi} \partial x^{m} \psi_{m}$ and $-i e^{\phi} G_{C}^{-R N S}$ in $Z$. Also, $G_{-3 / 2 C}^{-R N S} e^{\frac{i}{2} H_{C}^{R N S}}$ means the square root pole in the OPE of $G_{C}^{-R N S}$ with $e^{\frac{i}{2} H_{C}^{R N S}}$.

These operators now commute to give the spacetime supersymmetry algebra even offshell since $\left\{q_{a}^{\alpha}, q_{b}^{\beta}\right\}=-\frac{i}{2} \epsilon^{\alpha \beta} \oint \epsilon_{a b c d} \partial x^{c d}$. Having made a definite choice of pictures for all supersymmetry charges, it is also possible to make a definite choice of pictures for all vertex operators in a manifestly supersymmetric fashion. But doing this in a convenient manner requires information about the construction of the vertex operators that we will develop later.

For Type II strings, one can construct $N=2 D=6$ susy generators from the left and right-moving worldsheet fields. The additional generators coming from the right-moving worldsheet fields can be defined, just as in the the above lines, to be

$$
\begin{gathered}
\bar{q}_{\bar{a}}^{-}=\oint e^{-\frac{1}{2} \bar{\phi}} \bar{S}_{\bar{a}} e^{-\frac{i}{2} \bar{H}_{C}^{R N S}} \\
\bar{q}_{\bar{a}}^{+}=\oint\left(e^{\frac{3}{2} \bar{\phi}} \bar{b} \bar{\eta} \bar{S}_{\bar{a}} e^{\frac{i}{2} \bar{H}_{C}^{R N S}}-e^{\frac{1}{2} \bar{\phi}}\left(\frac{i}{2} \epsilon_{\bar{a} \bar{b} \bar{c}} \bar{S} \bar{b} \bar{\partial} x^{\bar{c} \bar{d}}+i \bar{S}_{\bar{a}} \bar{G}_{-3 / 2 C}^{-R N S}\right) e^{\frac{i}{2} \bar{H}_{C}^{R N S}} .\right.
\end{gathered}
$$

We are here using an unusual notation with the symbol $\bar{a}$ meant to cover the Type IIA and Type IIB cases simultaneously. For the Type IIA superstring, a lowered $\bar{a}$ index denotes a 
4 representation of $\mathrm{SU}(4)$ and a raised $\bar{a}$ index denotes a $\overline{4}$ representation of $\mathrm{SU}(4)$ (which is the opposite convention from that of the $a$ index). For the Type IIB superstring, a raised $\bar{a}$ index denotes a 4 representation of SU(4) and a lowered $\bar{a}$ index denotes a $\overline{4}$ representation of $\mathrm{SU}(4)$ (which is the same convention as that of the $a$ index).

These generators satisfy the $N=2 D=6$ susy algebra

$$
\left\{q_{a}^{\alpha}, q_{b}^{\beta}\right\}=\frac{1}{2} \epsilon^{\alpha \beta} \epsilon_{a b c d} P^{c d}, \quad\left\{\bar{q}_{\bar{a}}^{\alpha}, \bar{q}_{\bar{b}}^{\beta}\right\}=\frac{1}{2} \epsilon^{\alpha \beta} \epsilon_{\bar{a} \bar{b} \bar{c} \bar{d}} P^{\bar{c} \bar{d}} .
$$

The left and right moving supersymmetry algebras each admit $S U(2)$ groups of outer automorphisms, with generators $R^{\alpha \beta}$ and $\bar{R}^{\alpha \beta}$ satisfying the algebra

$$
\begin{aligned}
& {\left[R^{\alpha \beta}, q_{a}^{\gamma}\right]=\frac{1}{2}\left(\epsilon^{\beta \gamma} q_{a}^{\alpha}+\epsilon^{\alpha \gamma} q_{a}^{\beta}\right), \quad\left[\bar{R}^{\alpha \beta}, q_{a}^{\gamma}\right]=0,} \\
& {\left[R^{\alpha \beta}, \bar{q}_{\bar{a}}^{\gamma}\right]=0, \quad\left[\bar{R}^{\alpha \beta}, \bar{q}_{\bar{a}}^{\gamma}\right]=\frac{1}{2}\left(\epsilon^{\beta \gamma} \bar{q}_{\bar{a}}^{\alpha}+\epsilon^{\alpha \gamma} \bar{q}_{\bar{a}}^{\beta}\right) .}
\end{aligned}
$$

Although the supersymmetry algebra now closes off-shell, spacetime supersymmetry is not yet manifest in the RNS formalism since the worldsheet variables transform in a complicated manner under commutation with the susy generators defined in (4.5). In order to make susy manifest, one should therefore find a field redefinition of the worldsheet variables such that they transform in a simpler manner.

\subsection{Field redefinition to Green-Schwarz-like variables}

Supersymmetry generates translations of the odd coordinates of superspace. So the first step in defining the new variables is to look for a $\theta_{\alpha}^{a}$ variable which transforms as $\left\{q_{a}^{\alpha}, \theta_{\beta}^{b}\right\}=\delta_{a}^{b} \delta_{\beta}^{\alpha}$. This is easily done by defining

$$
\theta_{-}^{a}=e^{\frac{1}{2}\left(\phi+i H_{C}^{R N S}\right)} S^{a}, \quad \theta_{+}^{a}=c \xi e^{-\frac{1}{2}\left(3 \phi+i H_{C}^{R N S}\right)} S^{a} .
$$

However, it is easy to show that $\theta_{-}^{a}$ and $\theta_{+}^{a}$ are not independent fields since they satisfy $\theta_{-}^{a} \theta_{+}^{b}=\theta_{+}^{a} \theta_{-}^{b}$. In fact, one can write $\theta_{+}^{a}$ in terms of $\theta_{-}^{a}$ as $\theta_{+}^{a}=c \xi e^{-2 \phi-i H_{C}^{R N S}} \theta_{-}^{a}$, so one cannot choose all eight of these variables to be free fields. However, one can choose half of them, e.g. $\theta_{-}^{a}$, to be free fields. This breaks half of the eight manifest spacetime supersymmetries, but leaves four of them manifest. It might be possible to restore all eight supersymmetries by treating $\theta_{-}^{a}$ as a harmonic-like variable, but this has not yet been done. Since we will no longer refer to $\theta_{+}^{a}$, we shall rename $\theta_{-}^{a}=\theta^{a}$ for the rest of this paper. 
The conjugate momentum to $\theta^{a}$ is easily seen to be

$$
p_{a}=e^{-\frac{1}{2}\left(\phi+i H_{C}^{R N S}\right)} S_{a}
$$

whose zero mode is just the susy generator $q_{a}^{-}$. Note that $\theta^{a}$ and $p_{a}$ carry conformal weight 0 and 1 and satisfy the OPE

$$
p_{a}(y) \theta^{b}(z) \rightarrow \frac{\delta_{a}^{b}}{y-z} .
$$

Because of the $J_{C}^{R N S}$ dependence in $\theta^{a}$, $\theta^{a}$ has non-trivial OPE's with any RNS compactification variable $\chi_{C}^{R N S}$ carrying non-zero $\mathrm{U}(1)$ charge. For this reason, it is convenient to redefine the compactification variables to have no OPE singularities with the six-dimensional variables. To do so, we bosonize the $(\xi, \eta)$ variables as $\left(\xi=e^{-i \kappa}, \eta=e^{i \kappa}\right)$. Then we make a chiral $U(1)$ rotation of the compactification variables generated by the parameter $\phi+i \kappa-\frac{i}{2} \pi$. A field $\chi_{C}^{G S}$ that is a $U(1)$ primary of charge $n$ (so its dependence on $H_{C}^{R N S}$ is precisely $e^{i n H_{C}^{R N S}}$ ) is transformed to

$$
\chi_{C}^{G S}=e^{n\left(i \kappa+\phi-\frac{i}{2} \pi\right)} \chi_{C}^{R N S} .
$$

It is easy to check that the fields transformed in this way have no singular OPE's with $\theta^{a}$ or $p_{a}$ (and likewise with fields $\rho$ and $\sigma$ introduced below).

This chiral $\mathrm{U}(1)$ rotation of the compactification variables transforms the $N=$ 2 superconformal generators which describe the compactifaction. If $\left[T_{C}^{R N S}, G_{C}^{+} R N S\right.$, $\left.G^{-R N S}, J_{C}^{R N S}\right]$ are these generators, then the chiral rotation transforms these generators as

$$
\left[T_{C}^{G S}, G_{C}^{+G S}, G^{-G S}, J_{C}^{G S}\right] \rightarrow\left[e^{R} T_{C}^{R N S} e^{-R}, e^{R} G_{C}^{+R N S} e^{-R}, e^{R} G_{C}^{-R N S} e^{-R}, e^{R} J_{C}^{R N S} e^{-R}\right]
$$

where $R=\oint(i \kappa+\phi) J_{C}^{R N S}$. It is straightforward to show this implies that

$$
\begin{gathered}
T_{C}^{G S}=T_{C}^{R N S}+\partial^{2}(\phi+i \kappa)-\partial(\phi+i \kappa) J_{C}^{R N S}, \\
G_{C}^{+G S}=-i e^{\phi} \eta G_{C}^{+R N S}, \quad G_{C}^{-G S}=i e^{-\phi} \xi G_{C}^{-R N S}, \\
J_{C}^{G S}=J_{C}^{R N S}+2 \partial(\phi+i \kappa) .
\end{gathered}
$$

For example, for compactification on $T^{4}$,

$$
G^{+R N S}=\psi^{J R N S} \partial x^{\bar{J}}, \quad J_{C}^{R N S}=\psi^{J R N S} \psi^{\bar{J} R N S}
$$


is replaced with

$$
\begin{gathered}
G^{+G S}=\psi^{J G S} \partial x^{\bar{J}}=-i e^{\phi+i \kappa} \psi^{J R N S} \partial x^{\bar{J}}, \\
J_{C}^{G S}=\psi^{J}{ }^{G S} \psi^{\bar{J} G S}=\psi^{J R N S} \psi^{\bar{J} R N S}+2 \partial(\phi+i \kappa),
\end{gathered}
$$

where $x^{J}, x^{\bar{J}}, \psi^{J}, \psi^{\bar{J}}$ are the compactification variables and $J=1$ to 2 .

Not counting the compactification variables and the six $x^{m}$ 's, the original RNS theory contained two chiral bosons $(\beta, \gamma)$ and eight fermions $\left(\psi^{m}, b, c\right)$. Since the $\theta^{a}$ and $p_{a}$ variables describe eight fermions, one still needs to define two chiral bosons in the supersymmetric variables to recover the original degrees of freedom. These two chiral bosons should be defined such that they have no singular OPE's with the other Green-Schwarz-like variables. One of these chiral bosons, $\sigma$, is easily defined by bosonizing the $b, c$ ghosts as

$$
b=e^{-i \sigma}, \quad c=e^{i \sigma} .
$$

The remaining chiral boson, $\rho$, is defined by

$$
\rho=-2 \phi-i \kappa-i H_{C}^{R N S},
$$

which is the unique combination which has no singular OPE's with the other supersymmetric variables. These chiral bosons satisfy the standard OPE's

$$
\rho(y) \rho(z) \rightarrow-\log (y-z), \quad \sigma(y) \sigma(z) \rightarrow-\log (y-z) .
$$

The completeness of the new variables to the old ones (and the equivalence of the two descriptions) can be checked by bosonization of all variables. Using the conformal weights of the RNS fields, one can compute that the operator $e^{m \rho+i n \sigma}$ has conformal weight equal to $\frac{1}{2}\left(n^{2}-3 n-m^{2}+3 m\right)$. This operator has conformal weight zero when $n=m$. Note that typical $\rho$-dependent operators in the formalism are the real exponentials $e^{n \rho}$ with integer $n$; in this sense, $\rho$ is like the $\phi$ boson of the RNS formalism.

For the Type II superstring, all of the above definitions can be repeated for the rightmoving sector by simply placing bars over all fields. Ignoring the left and right-moving chiral bosons, the worldsheet action for the Green-Schwarz-like variables is given by

$$
S=\int d^{2} z\left(\frac{1}{2} \partial x^{m} \bar{\partial} x_{m}+p_{a} \bar{\partial} \theta^{a}+\bar{p}_{\bar{a}} \partial \bar{\theta}^{\bar{a}}\right)+S_{C}
$$

where $S_{C}$ is the action for the compactification variables. Note that $S_{C} \rightarrow S_{C}+\int d^{2} z \bar{\partial}(i \kappa+$ $\phi) J_{C}^{R N S}$ when one redefines the charged compactification variables as in (4.8). But since $\bar{\partial}(i \kappa+\phi)=0$, this redefinition does not affect $S_{C}$. 


\section{3. $N=2$ superconformal generators and $D=6$ spacetime-supersymmetry generators}

It is straightforward to write the RNS versions of the $N=2$ superconformal generators and $D=6$ spacetime-supersymmetry generators in terms of the Green-Schwarz-like variables defined in the previous subsection. As will be explained below, the $N=2$ superconformal generators of (3.1) get mapped after twisting (which in this case just removes the $\frac{1}{2} \partial(b c+\xi \eta)$ term from $\left.T\right)$ to

$$
\begin{gathered}
T=\frac{1}{2} \partial x^{m} \partial x_{m}+p_{a} \partial \theta^{a}+\frac{1}{2} \partial \rho \partial \rho+\frac{1}{2} \partial \sigma \partial \sigma-\frac{3}{2} \partial^{2}(\rho+i \sigma)+T_{C}^{G S} \\
G^{+}=-e^{-2 \rho-i \sigma}(p)^{4}+\frac{i}{2} e^{-\rho} p_{a} p_{b} \partial x^{a b}+ \\
e^{+i \sigma}\left(\frac{1}{2} \partial x^{m} \partial x_{m}+p_{a} \partial \theta^{a}+\frac{1}{2}(\partial \rho \partial \rho-\partial \sigma \partial \sigma)-\frac{1}{2} \partial^{2}(3 \rho+i \sigma)+T_{C}^{G S}\right) \\
+\partial^{2}\left(e^{i \sigma}\right)+\partial\left(e^{i \sigma}\left(\partial \rho+J_{C}^{G S}\right)\right)+G_{C}^{+G S}+e^{-2 \rho}(p)^{4} G_{C}^{-G S}, \\
G^{-}=e^{-i \sigma}, \\
J=\partial(\rho+i \sigma)+J_{C}^{G S}
\end{gathered}
$$

where $(p)^{4}=\frac{1}{24} \epsilon^{a b c d} p_{a} p_{b} p_{c} p_{d}$. The mapping of $T$ is easily found by noting that the supersymmetric variables are all free fields and by computing their conformal weights. The mapping of $G^{+}$contains various terms which come from the different terms in $j_{B R S T}$. For example, using the definitions of $p_{a}, \rho$ and $\sigma$ of the previous subsection, $(p)^{4}=e^{-2 \phi-2 i H_{C}^{R N S}}$ and $e^{2 \rho-i \sigma}=e^{4 \phi+2 i \kappa+2 i H_{C}^{R N S}} b$, so the first term in $G^{+}$is

$$
-(p)^{4} e^{2 \rho-i \sigma}=-e^{2 \phi+2 i \kappa} b=-\eta \partial \eta e^{2 \phi} b=-\gamma^{2} b .
$$

Similarly, the second term in $G^{+}$comes from the $-i \eta e^{\phi} \psi_{m} \partial x^{m}$ term in $\gamma G$, the third term comes from the $c T-b c \partial c$ term, the fourth and fifth terms come from the $\partial^{2} c+\partial(c \xi \eta)$ terms, and the sixth and seventh terms come from the $-i \eta e^{\phi}\left(G_{C}^{+R N S}+G_{C}^{-R N S}\right)$ term in $\gamma G$. The mapping of $G^{-}$comes from the bosonization of the $b$ ghost in (4.10). Finally, the mapping of $J$ comes from using (4.11) and (4.9) to show that $i \kappa=\partial \rho+J_{C}^{G S}$, so

$$
c b+\eta \xi=\partial(i \sigma+i \kappa)=\partial(i \sigma+\rho)+J_{C}^{G S} .
$$

The generators of (4.13) can be put in a more elegant form by performing the following similarity transformation on all Green-Schwarz-like variables: $y^{G S} \rightarrow e^{R} y^{G S} e^{-R}$ where

$$
R=\oint e^{i \sigma} G_{C}^{-} G S .
$$


For the rest of this paper, we will only refer to Green-Schwarz-like variables which have been transformed as in (4.14), and to simplify the notation, we will not change the symbol for these variables.

The similarity transformation of (4.14) preserves the free-field action of (4.12) since $e^{i \sigma} G_{C}^{-G S}$ is holomorphic. Furthermore, $e^{i \sigma} G_{C}^{-G S}$ is a $U(1)$-neutral primary field of conformal weight one, so $R$ commutes with $T$ and $J$. It is straightforward to compute that

$$
\begin{gathered}
{\left[R, G^{+}\right]=-e^{-2 \rho}(p)^{4} G_{C}^{-G S}+e^{2 i \sigma} G_{C}^{-G S}-e^{i \sigma} T_{C}^{G S}-\partial\left(e^{i \sigma} J_{C}^{G S}\right)-\partial^{2}\left(e^{i \sigma}\right), \quad\left[R, G^{-}\right]=G_{C}^{-G S}} \\
{\left[R,\left[R, G^{+}\right]\right]=-2 e^{2 i \sigma} G_{C}^{-G S}, \quad\left[R,\left[R,\left[R, G^{+}\right]\right]\right]=\left[R,\left[R, G^{-}\right]\right]=0}
\end{gathered}
$$

So after performing this similarity transformation, the above $N=2$ superconformal generators decompose into a $\hat{c}=0$ six-dimensional part and a $\hat{c}=2$ compactification-dependent piece as

$$
\begin{gathered}
T=\frac{1}{2} \partial x^{m} \partial x_{m}+p_{a} \partial \theta^{a}+\frac{1}{2} \partial \rho \partial \rho+\frac{1}{2} \partial \sigma \partial \sigma+\frac{3}{2} \partial^{2}(\rho+i \sigma)+T_{C}^{G S} \\
G^{+}=-e^{-2 \rho-i \sigma}(p)^{4}+\frac{i}{2} e^{-\rho} p_{a} p_{b} \partial x^{a b}+ \\
e^{+i \sigma}\left(\frac{1}{2} \partial x^{m} \partial x_{m}+p_{a} \partial \theta^{a}+\frac{1}{2} \partial(\rho+i \sigma) \partial(\rho+i \sigma)-\frac{1}{2} \partial^{2}(\rho+i \sigma)\right)+G_{C}^{+G S} \\
G^{-}=e^{-i \sigma}+G_{C}^{-G S} \\
J^{G S}=\partial(\rho+i \sigma)+J_{C}^{G S} .
\end{gathered}
$$

Finally, using these $N=2 \hat{c}=2$ generators, one can construct $\mathrm{N}=4$ generators in the way described in subsection (2.3). The additional $N=4$ generators are given by

$$
\begin{aligned}
& \widetilde{G}^{+}=e^{i H_{C}^{G S}+\rho}+e^{\rho+i \sigma} \widetilde{G}_{C}^{+G S}, \\
& \widetilde{G}^{-}=e^{-i H_{C}^{G S}}\left(-e^{-3 \rho-2 i \sigma}(p)^{4}+\frac{i}{2} e^{-2 \rho-i \sigma} p_{a} p_{b} \partial x^{a b}+\right. \\
& \left.e^{-\rho}\left(\frac{1}{2} \partial x^{m} \partial x_{m}+p_{a} \partial \theta^{a}+\frac{1}{2} \partial(\rho+i \sigma) \partial(\rho+i \sigma)-\frac{1}{2} \partial^{2}(\rho+i \sigma)\right)\right)+e^{-\rho-i \sigma} \widetilde{G}_{C}^{-G S}, \\
& J^{++}=e^{\rho+i \sigma} J_{C}^{++G S}, \\
& J^{--}=e^{-\rho-i \sigma} J_{C}^{--G S} \text {. }
\end{aligned}
$$

In terms of the Green-Schwarz-like variables, $\left[x^{m}, \theta^{a}, p_{a}, \rho, \sigma\right]$, the $D=6$ spacetimesupersymmetry generators of (4.5) drastically simplify to

$$
q_{a}^{-}=\oint p_{a}, \quad q_{a}^{+}=\oint\left(e^{-\rho-i \sigma} p_{a}-\frac{i}{2} \epsilon_{a b c d} \theta^{b} \partial x^{c d}\right) .
$$

Note that the term containing $G_{C}^{-R N S}$ in $q_{a}^{+}$was eliminated by the similarity transformation defined in (4.14). Since the compactification variables no longer appear in these generators, they are inert under susy transformations. 


\section{Massless Six-Dimensional Vertex Operators}

Given the formulas in (2.8), (4.16), and (4.17), the only missing element for computing manifestly spacetime-supersymmetric six-dimensional superstring amplitudes is explicit expressions for the vertex operators. After giving the definition of physical closed string vertex operators, we shall give explicit expressions for the massless vertex operators, first for those which are independent of the compactification fields, and then for those which depend on the compactification fields.

\subsection{Definition of Physical Vertex Operators}

As discussed in (2.6), physical vertex operators $\Phi^{+}$are defined for the open $N=4$ topological string by the conditions

$$
G_{0}^{+} \Phi^{+}=\widetilde{G}_{0}^{+} \Phi^{+}=\left(J_{0}-1\right) \Phi^{+}=0, \quad \Phi^{+} \sim \Phi^{+}+G_{0}^{+} \widetilde{G}_{0}^{+} \Lambda^{-}
$$

Since the $\widetilde{G}_{0}^{+}$cohomology is trivial in this case (this is easiest to check in RNS language where $\widetilde{G}^{+}=\eta$ ), it is always possible to define a $V$ satisfying

$$
\Phi^{+}=\widetilde{G}_{0}^{+} V, \quad G_{0}^{+} \widetilde{G}_{0}^{+} V=J_{0} V=0, \quad V \sim V+G_{0}^{+} \Lambda+\widetilde{G}_{0}^{+} \widetilde{\Lambda}
$$

It will be more convenient to describe the massless compactification-independent states in terms of the $\mathrm{U}(1)$-neutral vertex operator $V$, while it will be more convenient to describe the massless compactification-dependent states in terms of the U(1)-charged vertex operator $\Phi^{+}$. Using the relationship described above, it is straightforward to go from one type of vertex operator to the other.

The gauge-invariance of $V$,

$$
\delta V=G_{0}^{+} \Lambda+\widetilde{G}_{0}^{+} \widetilde{\Lambda}
$$

implies that one can choose the gauge-fixing conditions

$$
G_{0}^{-} V=\widetilde{G}_{0}^{-} V=0
$$

in a manner similar to the gauge-fixing of $b_{0}=0$ in the RNS formalism. Applying $G_{0}^{-} \widetilde{G}_{0}^{-}$to the equation of motion $G_{0}^{+} \widetilde{G}_{0}^{+} V=0$ in this gauge implies that $\left(T_{0}\right)^{2} V=0$, which implies that $T_{0} V=0$ since $T_{0}$ is just the conformal weight of $V$. Similarly, since $\Phi^{+}=\widetilde{G}^{+} V$,

$$
G_{0}^{-} \Phi^{+}=\widetilde{G}^{-} \Phi^{+}=T_{0} \Phi^{+}=0
$$


in this gauge.

As discussed in (3.5), the integrated form of the $\Phi^{+}$vertex operator is given by $\int G_{-1}^{-} \Phi^{+}$or $\int \widetilde{G}_{-1}^{-} \Phi^{+}$, where the two different choices are related by picture-changing. For the integrated form of the $V$ vertex operator, one has four different choices:

$$
\int G_{-1}^{-} G_{0}^{+} V, \quad \int \widetilde{G}_{-1}^{-} G_{0}^{+} V, \quad \int G_{-1}^{-} \widetilde{G}_{0}^{+} V, \quad \int \widetilde{G}_{-1}^{-} \widetilde{G}_{0}^{+} V
$$

but these four choices are also all related by picture-changing. The first two and last two choices are related for the same reason as in the $\Phi^{+}$case. The first and third choices (or second and fourth choices) are related by picture-changing for the following reason: If $\Phi^{+}=\widetilde{G}_{0}^{+} V$ is physical, then up to a gauge transformation $\delta V=\widetilde{G}_{0}^{+} \Lambda$ one has $V=\xi_{0} \Phi^{+}$ where $\xi_{0}$ is the $\xi$ zero mode and $G_{0}^{+} \Phi^{+}=\widetilde{G}_{0}^{+} \Phi^{+}=0$. Then $G_{0}^{+} V=Q \xi_{0} \Phi^{+}=Z \Phi^{+}$ is the picture-raised version of $\Phi^{+}$where $Z=\{Q, \xi\}$ is the picture-raising operator. So the integrated vertex operator $\int d z G_{-1}^{-} G_{0}^{+} V=\int d z G_{-1}^{-} Z \Phi^{+}$is related to the integrated vertex operator $\int d z G_{-1}^{-} \widetilde{G}_{0}^{+} V=\int d z G_{-1}^{-} \Phi^{+}$by replacing $\Phi^{+}$with its picture-raised $Z \Phi^{+}$. It will later be convenient to choose the picture of the integrated vertex operator to be the same as that of $V$, so we shall define the integrated vertex operator for $V$ to be the first choice of $(5.3)$, i.e.

$$
U=\int d z G_{-1}^{-} G_{0}^{+} V
$$

Physical gauge-fixed vertex operators for closed $N=4$ topological strings will be defined to be operators which satisfy the conditions of open $N=4$ string physical vertex operators in terms of both the left and right-moving constraints independently. 7 So the

7 This definition is naive since, in the usual closed string field theory, the off-shell closed string field is annihilated by $b_{0}-\bar{b}_{0}$. Furthermore, the on-shell closed string field is annihilated by $Q+\bar{Q}$, but not necessarily by $Q$ and $\bar{Q}$ independently. In practice, however, all physical vertex operators except for those describing special zero-momentum states are killed by $Q$ and $\bar{Q}$ independently. Also, one can use the $Q+\bar{Q}$ gauge-invariance to fix $b_{0}+\bar{b}_{0}=0$, which then combines with the off-shell $b_{0}-\bar{b}_{0}=0$ constraint to imply that $b_{0}=\bar{b}_{0}=0$. This is just the open string gauge-fixing condition imposed on both the left and right-moving sectors.

Although a field theory action for the the open $N=4$ topological string has been constructed [19], a field theory action for the closed $N=4$ topological string has not yet been constructed. So we cannot justify our closed string vertex operator conditions on the basis of an action principle, but are assuming that one can choose them to be the left-right product of the open string gaugefixed conditions of (5.1) and (5.2). It is possible that our conditions are too strong, which might imply that we are missing some special states. 
physical U(1)-charged vertex operators $\Phi^{++}$of the closed $N=4$ topological string must satisfy

$$
\begin{gathered}
G_{0}^{-} \Phi^{++}=\widetilde{G}_{0}^{-} \Phi^{++}=T_{0} \Phi^{++}=0, \\
\bar{G}_{0}^{-} \Phi^{++}=\overline{\widetilde{G}}_{0}^{-} \Phi^{++}=\bar{T}_{0} \Phi^{++}=0, \\
G_{0}^{+} \Phi^{++}=\widetilde{G}_{0}^{+} \Phi^{++}=\bar{G}_{0}^{+} \Phi^{++}=\overline{\widetilde{G}}_{0}^{+} \Phi^{++}=0 .
\end{gathered}
$$

Note that the last line of $(5.5)$ is implied by the other lines if $\Phi^{++}$happens to satisfy the condition $J_{0}^{++} \Phi^{++}=0$. This condition will be satisfied for the massless compactificationdependent vertex operator considered later in this section.

Similarly to the open superstring, one can construct a U(1)-neutral closed superstring vertex operator $V$ defined by $\Phi^{++}=\widetilde{G}_{0}^{+} \overline{\widetilde{G}}_{0}^{+} V$. Imposing the left-right product of the open string conditions of (5.1), $V$ must satisfy the conditions

$$
\begin{gathered}
G_{0}^{-} V=\widetilde{G}_{0}^{-} V=\bar{G}_{0}^{-} V=\overline{\widetilde{G}}_{0}^{-} V=T_{0} V=\bar{T}_{0} V=0, \\
G_{0}^{+} \widetilde{G}_{0}^{+} V=\bar{G}_{0}^{+} \overline{\widetilde{G}}_{0}^{+} V=0 .
\end{gathered}
$$

Note that the last line of (5.5) is implied by the other lines if $V$ happens to satisfy the condition $J_{0}^{++} \widetilde{G}_{0}^{+} V=\bar{J}_{0}^{++} \widetilde{G}_{0}^{+}=0$. This condition will be satisfied for the massless compactification-independent vertex operator considered later in this section. The integrated closed superstring vertex operator in terms of $V$ will be defined similar to the open superstring vertex operator of (5.4) as

$$
U=\int d^{2} z G_{-1}^{-} \bar{G}_{-1}^{-} G_{0}^{+} \bar{G}_{0}^{+} V .
$$

\subsection{Massless Compactification-Independent Vertex Operator}

For the closed superstring, the massless vertex operators which are independent of the compactification fields are most conveniently described by a $U(1)$-neutral vertex operator $V$ which must have conformal weight zero at zero momentum. The most general such vertex operator which is independent of the compactification fields is

$$
V=\sum_{m, n=-\infty}^{+\infty} e^{m(i \sigma+\rho)+n(i \bar{\sigma}+\bar{\rho})} V_{m, n}(x, \theta, \bar{\theta})
$$

where $V_{(m, n)}$ is any function of the zero modes of $x, \theta^{a}$, and $\bar{\theta}^{\bar{a}}$. It will now be shown that the physical conditions imply that $V$ describes the $N=(1,1) D=6$ supergravity 
multiplet for the Type IIA superstring, and describes the $N=(2,0) D=6$ supergravity multiplet plus an anti-self-dual tensor multiplet for the Type IIB superstring.

It is easy to check that $T_{0} V=0$ implies that $\partial^{p} \partial_{p} V_{m, n}=0$ and $G_{0}^{-} V=\bar{G}_{0}^{-} V=0$ implies that $V_{m, n}=0$ for $m>1$ or $n>1$. (For instance, $G_{0}^{-} V$ is the double pole in the $G^{-} \cdot V$ OPE, and as $G^{-}=e^{-i \sigma}$, it vanishes if $V_{m, n}=0$ for $m>1$.) This implies that $V$ satisfies $J_{0}^{++} \widetilde{G}_{0}^{+} V=0$ since $\widetilde{G}_{0}^{+} V=e^{i H_{C}^{G S}+2 \rho+i \sigma} V_{1,1}$. So the only remaining condition comes from $\widetilde{G}_{0}^{-} V=\overline{\widetilde{G}}_{0}^{-} V=0$. For convenience, let us define

$$
\widetilde{G}^{-}=A+B+C+D
$$

where

$$
\begin{gathered}
A=-e^{-3 \rho-2 i \sigma-i H_{C}^{G S}}(p)^{4}, \quad B=\frac{i}{2} e^{-2 \rho-i \sigma-i H_{C}^{G S}} p_{a} p_{b} \partial x^{a b}, \\
C=e^{-\rho-i H_{C}^{G S}}\left(\frac{1}{2} \partial x^{m} \partial x_{m}+p_{a} \partial \theta^{a}+\frac{1}{2} \partial(\rho+i \sigma) \partial(\rho+i \sigma)-\frac{1}{2} \partial^{2}(\rho+i \sigma)\right), \\
D=e^{-\rho-i \sigma} \widetilde{G}_{C}^{-G S} .
\end{gathered}
$$

When acting on $V_{1,1}, \widetilde{G}^{-}$has a double pole proportional to

$$
\begin{aligned}
{\left[e^{-2 \rho-i \sigma-i H_{C}^{G S}}(\partial(\right.} & \left.-3 \rho-2 i \sigma-i H_{C}^{(G S)}\right)(\nabla)^{4}-\frac{1}{6} \epsilon^{a b c d} p_{a} \nabla_{b} \nabla_{c} \nabla_{d} \\
+ & \left.\left.\frac{i}{2} e^{-\rho-i H_{C}^{G S}} \nabla_{a} \nabla_{b} \partial^{a b}\right)\right] V_{1,1}
\end{aligned}
$$

where the first two terms come from $A$ and the third term comes from $B$. We will use the notation that $\nabla_{a}=d / d \theta^{a}$ and $\bar{\nabla}_{\bar{a}}=d / d \bar{\theta}^{\bar{a}}$. The first and third terms of (5.8) imply that

$$
(\nabla)^{4} V_{1,1}=\nabla_{a} \nabla_{b} \partial^{a b} V_{1,1}=0
$$

however the second term can be cancelled by the double pole of $B$ on $V_{0,1}$ which is proportional to

$$
\frac{i}{2} e^{-2 \rho-i \sigma-i H_{C}^{G S}}\left(\partial\left(-2 \rho-i \sigma-i H_{C}^{G S}\right) \nabla_{a} \nabla_{b} \partial^{a b}+2 p_{a} \nabla_{b} \partial^{a b}+\partial x^{a b} \nabla_{a} \nabla_{b}\right] V_{0,1}
$$

This implies that

$$
\epsilon^{a b c d} \nabla_{b} \nabla_{c} \nabla_{d} V_{1,1}=6 i \nabla_{b} \partial^{a b} V_{0,1}, \quad \nabla_{a} \nabla_{b} V_{0,1}=0
$$

Elimination of double poles of $\widetilde{G}^{-}$on $V_{m, n}$ for $m<0$ implies that $V_{m, n}=0$ for $m<0$. 
Similarly, elimination of double poles of $\overline{\widetilde{G}}^{-}$with $V$ implies that

$$
\begin{gathered}
(\bar{\nabla})^{4} V_{1,1}=\bar{\nabla}_{\bar{a}} \bar{\nabla} \bar{b} \partial^{\bar{a} \bar{b}} V_{1,0}=0 \\
\epsilon^{\bar{a} \bar{b} \bar{c} \bar{d}} \bar{\nabla}_{\bar{b}} \bar{\nabla}_{\bar{c}} \bar{\nabla}_{\bar{d}} V_{1,1}=6 i \bar{\nabla}_{\bar{b}} \partial^{\bar{a} \bar{b}} V_{1,0}, \quad \bar{\nabla}_{\bar{a}} \bar{\nabla}_{\bar{b}} V_{1,0}=0
\end{gathered}
$$

and $V_{m, n}=0$ for $n<0$.

Furthermore, there is a residual gauge-invariance of $V$ coming from the fact that the physical conditions are preserved by the gauge transformation

$$
\delta V=G_{0}^{+} \Lambda+\bar{G}_{0}^{+} \bar{\Lambda}+\widetilde{G}_{0}^{+} \widetilde{\Lambda}+\overline{\widetilde{G}}_{0}^{+} \overline{\widetilde{\Lambda}}
$$

if the gauge parameters $\Lambda$ and $\widetilde{\Lambda}$ are annhilated by $\bar{G}_{0}^{+} \overline{\widetilde{G}}_{0}^{+}$, the gauge parameters $\bar{\Lambda}$ and $\overline{\widetilde{\Lambda}}$ are annihilated by $G_{0}^{+} \widetilde{G}_{0}^{+}$, and all gauge parameters are annihilated by $T_{0}, \bar{T}_{0}, G_{0}^{-}, \widetilde{G}_{0}^{-}$, $\bar{G}_{0}^{-}$, and $\overline{\widetilde{G}}_{0}^{-}$, Using this gauge invariance, one can gauge-fix the components of $V_{1,1}$ with no $\theta$ 's or no $\bar{\theta}$ 's by choosing the gauge parameters to be

$$
\Lambda=e^{-\rho-i H_{C}^{G S}+\bar{\rho}+i \bar{\sigma}} \lambda(x, \theta, \bar{\theta}), \quad \bar{\Lambda}=e^{2 \bar{\rho}+i \bar{\sigma}} \bar{\lambda}(x, \theta, \bar{\theta})
$$

which transforms

$$
\begin{gathered}
\delta V_{1,1}=\frac{i}{2} \nabla_{a} \nabla_{b} \partial^{a b} \lambda+\frac{i}{2} \bar{\nabla}_{\bar{a}} \bar{\nabla} \bar{b}_{\bar{b}} \partial^{\bar{a} \bar{b}} \bar{\lambda} \\
\delta V_{0,1}=(\nabla)^{4} \lambda, \quad \delta V_{1,0}=(\bar{\nabla})^{4} \bar{\lambda}
\end{gathered}
$$

Note that the usual residual gauge transformations of the graviton and NS-NS two-form,

$$
\delta\left(g_{m n}+b_{m n}\right)=\partial_{m} \xi_{n}+\partial_{n} \eta_{m}
$$

come from the gauge parameters

$$
\lambda=(\theta)^{4} \bar{\theta}^{\bar{a}} \bar{\theta}^{\bar{b}} \xi_{\bar{a} \bar{b}}, \quad \bar{\lambda}=\theta^{a} \theta^{b}(\bar{\theta})^{4} \eta_{a b}
$$

since the graviton and NS-NS two-form appear as

$$
\left(g_{m n}+b_{m n}\right) \sigma_{a b}^{m} \sigma_{\bar{c} \bar{d}}^{n} \theta^{a} \theta^{b} \bar{\theta}^{c} \bar{\theta}^{d}
$$

in $V_{1,1}$. 
Also, one can eliminate the remaining components of $V_{0, n}$ and $V_{n, 0}$ by using the gauge parameters

$$
\widetilde{\Lambda}=e^{-\rho-i H_{C}^{G S}+n(\bar{\rho}+i \bar{\sigma})} \widetilde{\lambda}_{n}(x, \theta, \bar{\theta}), \quad \overline{\widetilde{\Lambda}}=e^{-\bar{\rho}-i \bar{H}_{C}^{G S}+n(\rho+i \sigma)} \overline{\widetilde{\lambda}}(x, \theta, \bar{\theta}),
$$

which transforms $\delta V_{0, n}=\widetilde{\lambda}$ and $\delta V_{n, 0}=\overline{\widetilde{\lambda}}$.

The remaining degrees of freedom are described by the superfield

$$
\begin{gathered}
V_{1,1}=\theta^{a} \bar{\theta}^{\bar{a}} V_{a \bar{a}}^{--}+\theta^{a} \theta^{b} \theta^{\bar{a}} \sigma_{a b}^{m} \bar{\xi}_{m}^{-} \bar{a}^{a}+\theta^{a} \bar{\theta}^{\bar{a}} \bar{\theta}^{\bar{b}} \sigma_{\bar{a} \bar{b}}^{m} \xi_{m}^{-} a \\
+\theta^{a} \theta^{b} \bar{\theta}^{\bar{a}} \bar{\theta}^{\bar{b}} \sigma_{a b}^{m} \sigma_{\bar{a} \bar{b}}^{n}\left(g_{m n}+b_{m n}+\phi \eta_{m n}\right)+\theta^{a}\left(\bar{\theta}^{3}\right)_{\bar{a}} A_{a}^{-+} \bar{a}+\left(\theta^{3}\right)_{a} \bar{\theta}^{\bar{a}} A_{\bar{a}}^{+-a} \\
+\theta^{a} \theta^{b}\left(\bar{\theta}^{3}\right)_{\bar{a}} \sigma_{a b}^{m} \bar{\chi}_{m}^{+} \bar{a}+\left(\theta^{3}\right)^{a} \bar{\theta}^{\bar{a}} \bar{\theta}^{\bar{b}} \sigma_{\bar{a} \bar{b}}^{m} \chi_{m}^{+a}+\left(\theta^{3}\right)_{a}\left(\bar{\theta}^{3}\right)_{\bar{a}} F^{++a \bar{a}}
\end{gathered}
$$

This describes either the $\mathrm{N}=(1,1)$ supergravity multiplet for the Type IIA superstring, or the $\mathrm{N}=(2,0)$ supergravity multiplet plus an anti-self-dual tensor multiplet for the Type IIB superstring. The NS-NS fields are described by the symmetric traceless $g_{m n}$, the antisymmetric $b_{m n}$, and the dilaton $\phi$. The gravitino field is described by $\chi_{m}^{ \pm a}$ and $\bar{\chi}_{m}^{ \pm \bar{a}}$ where $\chi_{m}^{-a}=\partial^{a b} \xi_{b}^{-}$and $\bar{\chi}_{m}^{-\bar{a}}=\partial^{\bar{a} \bar{b}} \xi_{\bar{b}}^{-}$. The four Ramond-Ramond field strengths are described by $F^{ \pm \pm a \bar{a}}$ where

$$
F^{+-a \bar{a}}=\partial^{\bar{a} \bar{b}} A_{\bar{b}}^{+-a}, \quad F^{-+a \bar{a}}=\partial^{a b} A_{b}^{-+\bar{a}}, \quad F^{--a \bar{a}}=\partial^{a b} \partial^{\bar{a} \bar{b}} V_{b \bar{b}}^{--} .
$$

For the Type IIA superstring, the four Ramond-Ramond fields have spinor indices of opposite chirality and therefore describe the field-strengths of the four vectors in the $\mathrm{N}=(1,1)$ supergravity multiplet. For the Type IIB superstring, the four Ramond-Ramond fields have two spinor indices of the same chirality and therefore describe the field-strengths of four self-dual 2-forms and four scalars. The four Type IIB Ramond-Ramond self-dual 2forms combine with the NS-NS self-dual 2-form and the graviton to give the bosonic fields in the $\mathrm{N}=(2,0)$ supergravity multiplet. The remaining four Type IIB Ramond-Ramond scalars combine with the NS-NS dilaton and anti-self-dual 2-form to give the bosonic fields of an anti-self-dual tensor multiplet. Note that the pictures of the component fields of $V_{1,1}$ coincide with their conventional $\left(R^{+-}, \bar{R}^{+-}\right)$charge in supergravity (whose low-energy action is invariant under $\left.S U(2)_{R} \times S U(2)_{\bar{R}}\right)$.

Besides the mass-shell condition $\partial_{m} \partial^{m}=0$, these component fields satisfy

$$
\partial^{m} g_{m n}=\partial^{m} b_{m n}=0, \quad \partial^{m} \chi_{m}^{ \pm b}=\partial^{m} \bar{\chi}_{m}^{ \pm \bar{b}}=0
$$




$$
\partial_{c b} \chi_{m}^{ \pm b}=\partial_{\bar{c} \bar{b}} \bar{\chi}_{m}^{ \pm \bar{b}}=0, \quad \partial_{c b} F^{ \pm \pm b \bar{a}}=\partial_{\bar{c} \bar{b}} F^{ \pm \pm a \bar{b}}=0
$$

which come from component analysis of the superfield constraint

$$
\nabla_{a} \nabla_{b} \partial^{a b} V_{1,1}=\bar{\nabla}_{\bar{a}} \bar{\nabla} \bar{b} \partial^{\bar{a} \bar{b}} V_{1,1}=0
$$

The vertex operator in integrated form is given by

$$
\begin{gathered}
U=\int d^{2} z G_{1}^{-} \bar{G}_{1}^{-} G_{0}^{+} \bar{G}_{0}^{+} V \\
=\int d^{2} z\left|e^{-i \sigma-\rho} \frac{-\epsilon^{a b c d}}{6} p_{a}\left(\nabla_{b} \nabla_{c} \nabla_{d}\right)+i p_{a}\left(\nabla_{b} \partial^{a b}\right)+\frac{i}{2} \partial x^{a b}\left(\nabla_{a} \nabla_{b}\right)\right|^{2} V_{1,1} .
\end{gathered}
$$

So the vertex operators for $g_{m n}, b_{m n}$ and $\phi$ are proportional to the symmetric traceless, antisymmetric, and trace parts of

$$
\int d^{2} z\left(\partial x^{m}+\left(\sigma^{m p}\right)_{b}^{a} p_{a} \theta^{b} k_{p}\right)\left(\bar{\partial} x^{n}+\left(\sigma^{n q}\right)_{d}^{c} \bar{p}_{c} \bar{\theta}^{d} k_{q}\right) e^{i k \cdot x}
$$

while the vertex operator for $u_{i} \bar{u}_{j} F^{i j}{ }^{a \bar{a}}$ is proportional to 8

$$
\left.\int d^{2} z \mid u^{1}\left(-e^{-i \sigma-\rho} p_{a}-\frac{i}{2} \epsilon_{a b c d} \theta^{b} \partial x^{c d}+\frac{i}{2} \epsilon_{a b c d} \theta^{b} \theta^{c} k^{d e} p_{e}\right)+u^{2} p_{a}\right)\left.\right|^{2} e^{i k \cdot x} .
$$

\subsection{Massless Compactification-Dependent Vertex Operators}

The vertex operators for the massless moduli are constructed from the chiral-chiral $N=2$ worldsheet moduli of the compactification manifold, $P_{I}$, which satisfy

$$
\begin{gathered}
G^{+} P_{I}=\bar{G}^{+} P_{I}=\widetilde{G}^{+} P_{I}=\overline{\widetilde{G}}^{+} P_{I}=0, \\
J P_{I}=\bar{J} P_{I}=P_{I} .
\end{gathered}
$$

These vertex operators are most conveniently described by a $U(1)$-charged vertex operator $\Phi^{++}$whose compactification-independent part must contribute zero conformal weight at zero momentum. The most general such vertex operator is

$$
\Phi^{++}=\sum_{m, n=-\infty}^{+\infty} e^{m(i \sigma+\rho)+n(i \bar{\sigma}+\bar{\rho})} \Phi_{m, n}^{I}(x, \theta, \bar{\theta}) P_{I} .
$$

8 In the formula below, $u^{i}$ is simply a notational device for writing the $\mathrm{SU}(2)$ doublets using a single formula. However, it appears to be related to the $u^{i}$ s in (2.4) since $S U(2)_{\text {outer }}$ acts in a similar way to the $S U(2)$ of the $R^{j k}$ generators in (4.2). Understanding this relation could be useful for making all of the $D=6$ spacetime supersymmetries manifest. 
It is easy to check that $T_{0} \Phi^{++}=0$ implies that $\partial^{p} \partial_{p} \Phi^{++}=0$ and that $G_{0}^{-} \Phi^{++}=$ $\bar{G}_{0}^{-} \Phi^{++}=0$ implies that $\Phi_{m, n}=0$ for $m>1$ or $n>1$. Furthermore, $J_{0}^{++} \Phi^{++}=$ $\bar{J}_{0}^{++} \Phi^{++}=0$, so the only remaining conditions to be analyzed is $\widetilde{G}_{0}^{-} \Phi^{++}=\overline{\widetilde{G}}_{0}^{-} \Phi^{++}=0$. When acting on $\Phi_{1,1}, \widetilde{G}^{-}$has a quartic pole proportional to $(\nabla)^{4} \Phi_{1,1}^{I}$ and a triple pole with a term proportional to $\left(\nabla^{3}\right)^{a} \Phi_{1,1}^{I}$. When these are set to zero, $\widetilde{G}^{-}$still has a double pole coming from $A$ and $B$ of (5.7) which is proportional to

$$
\left[e^{-2 \rho-i \sigma}\left(-\frac{1}{4} \epsilon^{a b c d} p_{a} p_{b} \nabla_{c} \nabla_{d}\right)+i e^{-\rho} p_{a} \nabla_{b} \partial^{a b}\right] \Phi_{1,1}^{I} .
$$

The double pole coming from $B$ cannot be cancelled and implies that

$$
\nabla_{b} \partial^{a b} \Phi_{1,1}^{I}=0
$$

But the double pole coming from $A$ can be cancelled from the double pole of $B$ with $\Phi_{0,1}^{I}$, which is proportional to

$$
\frac{i}{2} e^{-2 \rho-i \sigma}\left(p_{a} p_{b} \partial^{a b}+2 p_{a} \partial x^{a b} \nabla_{b}\right) \Phi_{0,1}^{I},
$$

implying that

$$
\epsilon^{a b c d} \nabla_{c} \nabla_{d} \Phi_{1,1}^{I}=2 i \partial^{a b} \Phi_{0,1}^{I}, \quad \nabla_{a} \Phi_{0,1}^{I}=0 .
$$

Using similar arguments, one can show that $\widetilde{G}_{0}^{-} \Phi^{++}=0$ implies that

$$
\epsilon^{a b c d} \nabla_{c} \nabla_{d} \Phi_{1,0}^{I}=2 i \partial^{a b} \Phi_{0,0}^{I}, \quad \nabla_{a} \Phi_{0,0}^{I}=0
$$

and that $\Phi_{m, n}^{I}=0$ for $m<0$. Analogously, $\overline{\widetilde{G}}_{0}^{-} \Phi^{++}=0$ implies that

$$
\begin{gathered}
\bar{\nabla}_{\bar{b}} \partial^{\bar{a} \bar{b}} \Phi_{1,1}^{I}=\left(\bar{\nabla}^{3}\right)^{\bar{a}} \Phi_{1,1}^{I}=0, \\
\epsilon^{\bar{a} \bar{c} \bar{c} \bar{d}} \bar{\nabla}_{\bar{c}} \bar{\nabla}_{\bar{d}} \Phi_{1,1}^{I}=2 i \partial^{\bar{a} \bar{b}} \Phi_{1,0}^{I}, \quad \bar{\nabla}_{\bar{a}} \Phi_{1,0}^{I}=0, \\
\epsilon^{\bar{a} \bar{b} \bar{c} \bar{d}} \bar{\nabla}_{\bar{c}} \bar{\nabla}_{\bar{d}} \Phi_{0,1}^{I}=2 i \partial^{\bar{a} \bar{b}} \Phi_{0,0}^{I}, \quad \bar{\nabla}_{\bar{a}} \Phi_{0,0}^{I}=0 .
\end{gathered}
$$

There are no residual gauge invariances in this case, so the physical degrees of freedom are described by the superfields

$$
\begin{gathered}
\Phi_{1,1}^{I}=t_{++}^{I}+\theta^{a} \xi_{a}^{+I}+\bar{\theta}^{\bar{a}} \bar{\xi}_{\bar{a}}^{+I}+\theta^{a} \bar{\theta}^{\bar{a}} F_{a \bar{a}}^{I}+\theta^{a} \theta^{b} \partial_{a b} \Phi_{0,1}^{I}++\bar{\theta}^{\bar{a}} \bar{\theta}^{\bar{b}} \partial_{\bar{a} \bar{b}} \Phi_{1,0}^{I}, \\
\Phi_{0,1}^{I}=t_{-+}^{I}+\bar{\theta}^{\bar{a}} \bar{\xi}_{\bar{a}}^{-I}+\bar{\theta}^{\bar{a}} \bar{\theta}^{\bar{b}} \partial_{\bar{a} \bar{b}} t_{--}^{I},
\end{gathered}
$$




$$
\begin{gathered}
\Phi_{1,0}^{I}=t_{+-}^{I}+\theta^{a} \xi_{a}^{-I}+\theta^{a} \theta^{b} \partial_{a b} t_{--}^{I}, \\
\Phi_{0,0}^{I}=t_{--}^{I} .
\end{gathered}
$$

These describe the vector (or tensor) multiplets coming from the Type IIA (or Type IIB) compactification. For example, the four NS-NS scalar moduli are described by $t_{ \pm \pm}^{I}$, the modulinos are described by $\xi_{a}^{ \pm I}$ and $\bar{\xi}_{\bar{a}}^{ \pm I}$, while the Ramond-Ramond field-strength is described by $F_{a \bar{a}}^{I}$. Besides the mass-shell condition $\partial_{m} \partial^{m}=0$, these fields satisfy $\partial^{a b} \xi_{a}^{ \pm I}=\partial^{\bar{a} \bar{b}} \bar{\xi}_{\bar{a}}^{ \pm I}=\epsilon^{a b c d} \partial_{b c} F_{a \bar{a}}^{I}=\epsilon^{\bar{a} \bar{b} \bar{c} \bar{d}} \partial_{\bar{b} \bar{c}} F_{a \bar{a}}^{I}=0$, which comes from the superfield constraint $\nabla_{b} \partial^{a b} \Phi_{1,1}^{I}=\bar{\nabla}_{\bar{b}} \partial^{\bar{a} \bar{b}} \Phi_{1,1}^{I}=0$. For Type IIA (or Type IIB) compactifications, $F_{a \bar{a}}^{I}$ describes the field-strength of a vector (or a self-dual tensor plus a scalar).

The vertex operator in integrated form is given by

$$
\begin{gathered}
U=\int d^{2} z G_{1}^{-} \bar{G}_{1}^{-} \Phi^{++} \\
=\int d^{2} z\left[\left(\left|e^{i \sigma+\rho}\right|^{2} \Phi_{1,1}^{I}+e^{i \sigma+\rho} \Phi_{1,0}^{I}+e^{i \bar{\sigma}+\bar{\rho}} \Phi_{0,1}^{I}+\Phi_{0,0}^{I}\right)\left(G_{1}^{-} \bar{G}_{1}^{-} P_{I}\right)\right. \\
+\left(e^{\rho+i \bar{\sigma}+\bar{\rho}} \Phi_{1,1}^{I}+e^{\rho} \Phi_{1,0}^{I}\right)\left(\bar{G}_{1}^{-} P_{I}\right) \\
+\left(e^{\rho+i \sigma+\bar{\rho}} \Phi_{1,1}^{I}+e^{\bar{\rho}} \Phi_{0,1}^{I}\right)\left(G_{1}^{-} P_{I}\right) \\
\left.+e^{\rho+\bar{\rho}} \Phi_{1,1}^{I} P_{I}\right] .
\end{gathered}
$$

The vertex operator for the component fields are easily computed from the $\theta$ expansion of $U$. For example, the vertex operator for $t_{--}^{I}$ is

$$
\left.\int d^{2} z \mid e^{i \sigma+\rho} \theta^{a} \theta^{b} k_{a b}+1\right) G_{1}^{-}+\left.e^{\rho} \theta^{a} \theta^{b} k_{a b}\right|^{2} P_{I} e^{i k \cdot x}
$$

and for $t_{++}^{I}$ is

$$
\int d^{2} z\left|e^{i \sigma+\rho} G_{1}^{-}+e^{\rho}\right|^{2} P_{I} e^{i k \cdot x}
$$

The vertex operator for the Ramond-Ramond field-strength $F_{a \bar{a}}^{I}$ is

$$
\int d^{2} z \theta^{a} \bar{\theta}^{\bar{a}}\left|e^{i \sigma+\rho} G_{1}^{-}+e^{\rho}\right|^{2} P_{I} e^{i k \cdot x}
$$

Note that replacing $G^{-}$with $\widetilde{G}^{-}$in the definition of $U$ only changes the 'picture' and therefore does not provide new physical states. One therefore does not need to consider vertex operators constructed from chiral/anti-chiral or anti-chiral/anti-chiral moduli of the compactification manifold. 


\section{Almost Flat Approximation to the $\mathrm{AdS}_{3} \times \mathrm{S}^{3}$ Background}

\subsection{Linearized Perturbation}

As discussed in (4.12), the six-dimensional part of the superstring action in a Minkowski background is proportional to

$$
S_{0}=\int d^{2} \sigma\left[\frac{1}{8} \epsilon_{a b c d} \partial x^{a b} \bar{\partial} x^{c d}+p_{a} \bar{\partial} \theta^{a}+\bar{p}_{a} \partial \bar{\theta}^{a}\right] .
$$

where $\partial=\frac{\partial}{\partial \sigma_{1}}-i \frac{\partial}{\partial \sigma_{2}}$ and $\bar{\partial}=\frac{\partial}{\partial \sigma_{1}}+i \frac{\partial}{\partial \sigma_{2}}$. Here we have substituted $x^{a b}=x^{m}\left(\sigma_{m}\right)^{a b}$ and have omitted the chiral boson action for $\rho$ and $\sigma$, which are free fields.

We now want to make a small deformation of this free-field action, adding the vertex operators of a suitable self-dual RR three-form and graviton, so as to describe an $\mathrm{AdS}_{3} \times \mathbf{S}^{3}$ background that is almost flat. This is appropriate for describing an $\operatorname{AdS}_{3} \times \mathbf{S}^{3}$ background obtained by compactification on $\mathrm{K} 3$ or $\mathbf{T}^{4}$ with large values of the onebrane and fivebrane charges $Q_{1}$ and $Q_{5}$. By studying the almost flat case, we will get clues about how $\operatorname{AdS}_{3} \times \mathbf{S}^{3}$ should be described in general in the framework we are using here, and this will make possible a more complete description in section 7 .

We begin by describing the explicit perturbation that is needed to get from $\mathbf{R}^{6}$ to $\mathrm{AdS}_{3} \times \mathbf{S}^{3}$ in the almost flat case. The symmetry group of $\mathbf{R}^{6}$ is, of course, the six-dimensional Poincaré group, and the subgroup that leaves fixed a point is the sixdimensional rotation group $S O(6)$ (or $S O(5,1)$ if we use Lorentz signature). The subgroup of the symmetry group of $\mathrm{AdS}_{3} \times \mathbf{S}^{3}$ that leaves fixed a point is $S O(3) \times S O(3)$ (or $S O(2,1) \times S O(3)$ if we use Lorentz signature), since one can make $S O(3)$ rotations about any given point in the homogeneous, isotropic spaces $\mathrm{AdS}_{3}$ and $\mathbf{S}^{3}$, but there are not rotations that mix the two.

The reason to focus on the symmetries that fix a given point is the following. No matter how small the cosmological constant may be, $\mathrm{AdS}_{3} \times \mathbf{S}^{3}$, if one looks at it "in the large," is not a small perturbation of $\mathbf{R}^{6}$. The $\mathbf{S}^{3}$ is compact, for example, and the deviation of $\mathrm{AdS}_{3}$ from flat $\mathbf{R}^{3}$ grows as one goes to infinity. Viewing $\mathrm{AdS}_{3} \times \mathbf{S}^{3}$ as a perturbation of $\mathbf{R}^{6}$ is a local process; for small cosmological constant, a portion of $\mathbf{R}^{6}$ near a given point, say the origin $P$, can be regarded as an approximation to a portion of $\mathrm{AdS}_{3} \times \mathbf{S}^{3}$. In view of what was said in the last paragraph, the perturbation that goes from $\mathbf{R}^{6}$ to $\mathrm{AdS}_{3} \times \mathbf{S}^{3}$ must break the $S O(6)$ group of rotations around $P$ to $S O(3) \times S O(3)$.

In the flat case, our notation was adapted to the local isomorphism $S O(6) \cong S U(4)$. The $S O(3) \times S O(3)$ subgroup of $S O(6)$ is instead locally isomorphic to an $S O(4)$ subgroup 
of $S U(4)$, under which the $\mathbf{4}$ and $\overline{\mathbf{4}}$ of $S U(4)$ become isomorphic and transform as the $\mathbf{4}$ of $S O(4)$. We can usefully proceed with much the same notation as before, with one crucial difference that in $S O(4)$ there is an invariant metric tensor $\delta_{a b}$, which can be used to raise and lower indices.

A self-dual two-form $H$ that preserves an $S O(3) \times S O(3)$ group of rotations must at $P$ be of the form

$$
-i H_{012}=H_{345}=2 f
$$

for some real constant $f$. Here we identify $x^{0}, x^{1}, x^{2}$ as $\mathrm{AdS}_{3}$ coordinates, and $x^{4}, x^{5}, x^{6}$ as $\mathbf{S}^{3}$ coordinates. Note that in Euclidean signature the self-duality condition reads $H=i * H$, which is the reason for the $i$ in (6.1). A Wick rotation on the $x^{0}$ coordinate (taking us to Lorentz signature for $\mathrm{AdS}_{3}$ ) will make $H$ real. $9 \mathrm{AdS}_{3} \times \mathbf{S}^{3}$ also has "broken" symmetries that do not leave $P$ fixed. In the almost flat approximation, these correspond to the translations of $\mathbf{R}^{6}$, and invariance under them says that $H_{012}$ and $H_{345}$ should be constant.

We want to embed this $H$ field in the six-dimensional Type IIB supergravity multiplet for which vertex operators were constructed in the last section. As we have seen, this multiplet contains the four RR tensor fields $H^{ \pm \pm}$. There is also an NS tensor field. However, we will begin by considering the case of a perturbation by an RR tensor field, and will extend the discussion to the NS perturbation only at the end of the present section.

The low energy supergravity in six dimensions has an $S O(5) \mathrm{R}$ symmetry group. The subgroup of $S O(5)$ that does not mix RR and NS worldsheet vertex operators is $S O(4) \cong$ $S U(2) \times S U(2)$, where one $S U(2)$ acts on the first \pm index of $H^{ \pm \pm}$, and the other acts on the second. The four fields $H^{ \pm \pm}$thus transform as a vector of $S U(2) \times S U(2) \cong S O(4)$, so if $H$ is real, it can be rotated into any desired "direction" by an $S U(2) \times S U(2)$ transformation. (By $H$ being real we mean of course that it is real if the spactime signature is taken to be Lorentzian.) If we denote $H^{ \pm \pm}$as $H^{\alpha \alpha^{\prime}}$, where $\alpha, \alpha^{\prime}=1,2$ label the two-dimensional representations of the two $S U(2)$ 's, then the reality condition on $H$ is $H^{\alpha \alpha^{\prime}}=\epsilon^{\alpha \beta} \epsilon^{\alpha^{\prime} \beta^{\prime}} \bar{H}_{\beta \beta^{\prime}}$. So a representative example of a real field is a field with $H^{++}$and $H^{--}$equal and real. Hence with no essential loss of generality, we take $H^{++}=H^{--}=H / 2$, with $H$ as above.10

$9 H$ is also real if the signature is $(3,3)$, a case that is natural in group theory though not in physics, as we will see in the next section.

10 Since the $S U(2) \times S U(2)$ mentioned in this paragraph is not a symmetry of the string theory, we should explain more accurately in what sense we may rotate $H$ by $S U(2) \times S U(2)$. The point is that the conformal field theory of the compactification manifold $\mathrm{K} 3$ or $\mathrm{T}^{4}$ actually has $(4,4)$ 
From what we have seen in section 4 , the vertex operators for $H^{--}$and $H^{++}$are $p^{a} \bar{p}^{a^{\prime}}$ and $\left(p^{a} e^{\phi}-\frac{i}{2} \epsilon_{a b c d} \theta^{b} \partial x^{c d}\right)\left(\bar{p}^{a^{\prime}} e^{\phi}-\frac{i}{2} \epsilon_{a^{\prime} b^{\prime} c^{\prime} d^{\prime}} \bar{\theta}^{b^{\prime}} \bar{\partial} x^{c^{\prime} d^{\prime}}\right)$. To get the precise $H$ field of eqn. (6.1), we must combine the left and right-moving parts of the vertex operators in a way that is invariant under an $S O(4)$ subgroup in which the $p$ 's and $\theta$ 's transform as a vector. This is done just by contracting with $\delta^{a a^{\prime}}$, so the desired vertex operator is

$$
V_{H}^{R R}=f \int d^{2} \sigma\left[p^{a} \bar{p}^{a}+\left(p^{a} e^{\phi}-\frac{i}{2} \epsilon_{a b c d} \theta^{b} \partial x^{c d}\right)\left(\bar{p}^{a} e^{\bar{\phi}}-\frac{i}{2} \epsilon_{a b^{\prime} c^{\prime} d^{\prime}} \bar{\theta}^{b^{\prime}} \bar{\partial} x^{c^{\prime} d^{\prime}}\right)\right]
$$

where $\phi=-\rho-i \sigma$ and $\bar{\phi}=-\bar{\rho}-i \bar{\sigma}$. Here $f$ is a small parameter measuring the strength of the perturbation. This vertex operator is just the sum of the "squares" of the two spacetime susy generators defined in (4.18).

Note that even after adding such an interaction term to the Lagrangian, $e^{\phi}=e^{-\rho-i \sigma}$ is still a chiral field of dimension zero, as its operator product singularity with itself (and hence with $V_{H}^{R R}$ ) vanishes. Other combinations of $\rho$ and $\sigma$ such as $e^{\rho-i \sigma}$ are no longer chiral.

To order $f$, the deformation to $\mathrm{AdS}_{3} \times \mathbf{S}^{3}$ is simply made by adding $V_{H}$ to the Lagrangian. In order $f^{2}$, we must expect additional corrections. For example, the Einstein equations read roughly $R_{a b}=\left(H^{2}\right)_{a b}$, so the departure of $\mathrm{AdS}_{3} \times \mathbf{S}^{3}$ from flatness is in order $f^{2}$. Near $P$, which we identify as the point $x=0$, the $\operatorname{AdS}_{3} \times \mathbf{S}^{3}$ background is described by the metric

$$
g_{m n}=\eta_{m n}+\frac{1}{6} f^{2}\left(\sigma_{m}\right)^{a e}\left(\sigma_{n}\right)^{b f} \epsilon_{a b c d} x^{c e} x^{d f}+O\left(f^{4}\right),
$$

where $f$ is the same constant as in the three-form. The vertex operator for the order $f^{2}$ deformation cannot be constructed just using the results of section 5 ; in that section, we considered metric perturbations of flat empty space obeying $R_{a b}=0$, while now the relevant equation is $R_{a b}=\left(H^{2}\right)_{a b}$. Nonetheless, it is reasonable to expect the $O\left(f^{2}\right)$ terms in the action to have the form

$$
V_{g}=f^{2} \int d^{2} \sigma\left[\frac{1}{24} \epsilon_{a b c d} x^{a e} x^{b f} \partial x^{c e} \bar{\partial} x^{d f}-\ldots\right]
$$

with the $\ldots$ terms involving $\theta$-dependent couplings such as $p \partial \theta x \partial x$. We will determine the details of the additional couplings in section 7 using spacetime supersymmetry, but

worldsheet supersymmetry, but we viewed it as a $(2,2)$ theory in the flat space construction in sections 4 and 5 . By rotating the choice of $(2,2)$ embedding in $(4,4)$, we rotate what we mean by $H^{++}$and $H^{--}$. 
for now we leave them undetermined and analyze some general properties that will give us important clues for later.

Already at this level, the problems of principle of adding RR fields to the vacuum, in the RNS formalism, have been circumvented. There are no spin fields in the Lagrangian that might make computations difficult, and no ambiguity coming from the different pictures in which an RR field can be written, since a consistent choice of pictures has been made. The price we pay for these simplifications is that - in contrast to more familiar worldsheet actions in the RNS formalism, or the flat case that we reviewed in the present formalism in section 4 - there are couplings of the "matter" fields $x, \theta$, and $p$ to the "ghost" fields $\phi, \bar{\phi}$. What saves the day is that the ghost couplings have a very simple structure. Because the Lagrangian only has positive powers of $e^{\phi}$ and $e^{\bar{\phi}}$ (a fact that reflects the fact that the vertex operators have this property, and hence will persist in higher orders), the ghost couplings are rather like "screening charges"; in any given computation of spectrum or perturbative scattering amplitudes, they can be treated as infinitesimal perturbations, and taken into account only up to some finite order.

If one performs the rescaling 11

$$
\begin{aligned}
p_{a} \rightarrow f^{-\frac{1}{2}} p_{a}, \quad \bar{p}_{a} & \rightarrow f^{-\frac{1}{2}} \bar{p}_{a}, \quad \theta^{a} \rightarrow f^{+\frac{1}{2}} \theta^{a}, \quad \bar{\theta}^{a} \rightarrow f^{+\frac{1}{2} \bar{\theta}^{a},} \\
e^{\phi} & \rightarrow-\frac{f}{4} e^{\phi}, \quad e^{\bar{\phi}} \rightarrow-\frac{f}{4} e^{\bar{\phi}},
\end{aligned}
$$

then the action $S_{A d S}=S_{0}+V_{H}+V_{g}$ becomes

$$
\begin{aligned}
S_{A d S}= & \int d^{2} \sigma\left[\frac{1}{8} \epsilon_{a b c d} \partial x^{a b} \bar{\partial} x^{c d}+p_{a} \bar{\partial} \theta^{a}+\bar{p}_{a} \partial \bar{\theta}^{a}+p^{a} \bar{p}^{a}\right. \\
& +f^{2}\left(\left(-\frac{1}{4} p^{a} e^{\phi}-\frac{i}{2} \epsilon^{a b c d} \theta^{b} \partial x^{c d}\right)\left(-\frac{1}{4} \bar{p}^{a} e^{\bar{\phi}}-\frac{i}{2} \epsilon^{a b^{\prime} c^{\prime} d^{\prime}} \bar{\theta}^{b^{\prime}} \bar{\partial} x^{c^{\prime} d^{\prime}}\right)\right. \\
& \left.\left.+\frac{1}{24} \epsilon_{a b c d} x^{a e} x^{b f} \partial x^{c e} \bar{\partial} x^{d f}+\ldots\right)\right] .
\end{aligned}
$$

This exhibits $f$ as a "coupling constant"; the kinetic terms are independent of $f$, and the interaction are all of order $f^{2}$. Since the interaction terms are homogeneous and quartic in the variables $x, p, \theta, e^{\phi}, \ldots$ a further rescaling

$$
\left(x, p, \theta, e^{\phi}, \bar{p}, \bar{\theta}, e^{\bar{\phi}}\right) \rightarrow f^{-1}\left(x, p, \theta, e^{\phi}, \bar{p}, \bar{\theta}, e^{\bar{\phi}}\right)
$$

11 Note that the rescaling of $e^{\phi}$ and $e^{\bar{\phi}}$ in the following formula amounts to the addition of a constant to the fields $\phi, \bar{\phi}$, and so is a symmetry of the free action of these fields. The factor of $-\frac{1}{4}$ is included to simplify comparison with the exact non-linear action. 
puts the action in a form in which $f$ appears only as an overall multiplicative constant:

$$
\begin{aligned}
& S_{A d S}^{\prime}=\frac{1}{f^{2}} \int d^{2} \sigma\left[\frac{1}{8} \epsilon^{a b c d} \partial x_{a b} \bar{\partial} x_{c d}+p_{a} \bar{\partial} \theta^{a}+\bar{p}_{a} \partial \bar{\theta}^{a}+p^{a} \bar{p}^{a}\right. \\
& +\left(-\frac{1}{4} p^{a} e^{\phi}-\frac{i}{2} \epsilon^{a b c d} \theta^{b} \partial x^{c d}\right)\left(-\frac{1}{4} \bar{p}^{a} e^{\bar{\phi}}-\frac{i}{2} \epsilon^{a b^{\prime} c^{\prime} d^{\prime}} \bar{\theta}^{b^{\prime}} \bar{\partial} x^{c^{\prime} d^{\prime}}\right) \\
& \left.+\frac{1}{24} \epsilon_{a b c d} x^{a e} x^{b f} \partial x^{c e} \bar{\partial} x^{d f}+\ldots\right] \text {. }
\end{aligned}
$$

From this point of view, the expansion we are making around the flat case is an expansion in powers of the fields, which we consider small; $S_{A d S}^{\prime}$ is the $\operatorname{AdS}_{3} \times \mathbf{S}^{3}$ action up to fourth order in the fields, except that some of the back reaction terms have not yet been determined.

\subsection{Sigma Model With Supermanifold As Target}

Now we meet a crucial fact which substantially changes the character of the problem from what we have seen up to this point, and leads to one of the main insights of the present paper. In the flat case, the Lagrangian is linear in $p$ and has the typical structure "second order in bosons, first order in fermions," of most physical supersymmetric actions. But now, in (6.6), we see that there is a $\bar{p} p$ term, and moreover that (at least perturbatively in the fields) its coefficient is everywhere nonzero. Hence $p$ and $\bar{p}$ can be integrated out to give a Lagrangian for $x, \theta$, and $\bar{\theta}$ only (plus couplings to the "ghosts" $\phi, \bar{\phi}$ ).

Solving for the equation of motion for $p^{a}$ and $\bar{p}^{a}$, one gets the action

$$
\begin{aligned}
S_{A d S}^{\prime}=\frac{1}{f^{2}} \int d^{2} \sigma[ & \frac{1}{8} \epsilon^{a b c d} \partial x_{a b} \bar{\partial} x_{c d}-\bar{\partial} \theta^{a} \partial \bar{\theta}^{a} \\
& +\frac{i}{8} \epsilon^{a b c d}\left(e^{\phi} \partial \bar{\theta}^{a} \bar{\theta} b \bar{\partial} x^{c d}+e^{\bar{\phi}} \bar{\partial} \theta^{a} \theta^{b} \partial x^{c d}\right) \\
& \left.+\frac{1}{24} \epsilon_{a b c d} x^{a e} x^{b f} \partial x^{c e} \bar{\partial} x^{d f}+\ldots\right] .
\end{aligned}
$$

Now let us try to interpret this Lagrangian. The spacetime supersymmetry generators, constructed in (4.18), depend on $\phi$ and $\bar{\phi}$, but only via positive powers of $e^{\phi}$ and $e^{\bar{\phi}}$. There likewise are only positive powers of $e^{\phi}$ and $e^{\bar{\phi}}$ in $S_{A d S}$. Hence, the part of $S_{A d S}^{\prime}$ that is independent of $\phi$ and $\bar{\phi}$ must be spacetime supersymmetric by itself. Let us call this action $\widehat{S}_{A d S}$.

$\widehat{S}_{A d S}$ has some properties that are not obvious from its origin via a small perturbation of the flat model. First of all, there is a continuous $S U(2)$ symmetry under which $\theta^{a \alpha}=$ 
$\left(\theta^{a}, \bar{\theta}^{a}\right)$ transforms as a doublet (for each fixed value of the $S O(4)$ index $a$ ). To write the action in a manifestly $S U(2)$-invariant way, we introduce the antisymmetric tensor $\epsilon_{\alpha \beta}$ with $\epsilon_{\alpha \beta} \theta^{a \alpha} \theta^{b \beta}=\theta^{a} \bar{\theta}^{b}+\theta^{b} \bar{\theta}^{a}, \epsilon^{\alpha \beta} \epsilon_{\beta \gamma}=\delta_{\gamma}^{\alpha}$. We furthermore note that $S_{A d S}^{\prime}$ (and all previous actions written in this section) has a manifest symmetry under $z \leftrightarrow \bar{z}, \theta \rightarrow \bar{\theta}$, $\bar{\theta} \rightarrow-\theta$. Since $\theta \rightarrow \bar{\theta}, \bar{\theta} \rightarrow-\theta$ is an $S U(2)$ transformation, the fact that the action has $S U(2)$ symmetry means that this operation itself (without $z \leftrightarrow \bar{z}$ ) is a symmetry, and hence that there is also symmetry under $z \leftrightarrow \bar{z}$, with no action on the fields. Hence we make a further change of notation to exhibit this symmetry, which we will call worldsheet parity. To do so, we introduce real coordinates $\sigma^{1}, \sigma^{2}$, with $z=\frac{1}{2}\left(\sigma^{1}+i \sigma^{2}\right)$. With these choices, we can write $\widehat{S}_{A d S}$ in the form

$$
\begin{aligned}
\widehat{S}_{A d S}= & \frac{1}{f^{2}} \int d^{2} \sigma\left(\frac{1}{8} \epsilon_{a b c d} \partial_{i} x^{a b} \partial_{i} x^{c d}-\frac{1}{2} \partial_{i} \theta^{a \alpha} \partial^{i} \theta^{a \beta} \epsilon_{\alpha \beta}\right. \\
& \left.+\frac{1}{24} \epsilon_{a b c d} x^{a e} x^{b f} \partial_{i} x^{c e} \partial_{i} x^{d f}+\ldots\right)
\end{aligned}
$$

which is manifestly invariant under $S U(2)$ and also under worldsheet parity. The $S U(2)$ symmetry is not yet so striking at this point, because the dependence on $\theta$ and $\bar{\theta}$ is so simple and we have not determined the ... terms. However, we will see in section 7 that the full sigma model action (modulo ghost couplings) does have the $S U(2)$ symmetry.

What is $\widehat{S}_{A d S}$ ? The kinetic energy is quadratic for all bose and fermi fields. Thus, $\widehat{S}_{A d S}$ is a "sigma model with a supermanifold as target space." The action, in other words, is of the general form $\int d^{2} \sigma g_{I J} \partial_{i} \Phi^{I} \partial^{i} \Phi^{J}$, with bosonic and fermionic coordinates $\Phi^{I}, \Phi^{J}$ and metric $g_{I J}$ on a target supermanifold $M$. The coordinates are in fact $x^{m n}$ and $\theta^{a \alpha}$, so the bosonic and fermionic dimension of the target space is $6 \mid 8$. The worldsheet parity symmetry of $\widehat{S}_{A d S}$ means that there is no "Wess-Zumino term." In this context, a Wess-Zumino term would be a term of the general form $\int d^{2} \sigma \epsilon^{i j} B_{I J} \partial_{i} \Phi^{I} \partial_{j} \Phi^{J}$, with $B_{I J}$ a two-form on $M$.

Moreover, spacetime supersymmetry acts on the sigma model in the most elementary way: by geometrical transformations of $M$. Recall that in the flat Minkowski background, the spacetime supersymmetry generators were given by

$$
\begin{aligned}
& q_{a}^{-}=\oint p_{a}, \quad q_{a}^{+}=\oint\left(e^{\phi} p_{a}-\frac{i}{2} \epsilon_{a b c d} \theta^{b} \partial x^{c d}\right), \\
& \bar{q}_{a}^{-}=\oint \bar{p}_{a}, \quad q_{a}^{+}=\oint\left(e^{\bar{\phi}} \bar{p}_{a}-\frac{i}{2} \epsilon_{a b c d} \bar{\theta}^{b} \bar{\partial} x^{c d}\right) .
\end{aligned}
$$


Replacing $p^{a}$ with $\partial \bar{\theta}^{a}$ and $\bar{p}^{a}$ with $-\bar{\partial} \theta^{a}$, and using the OPE's

$$
x^{a b}(y) x^{c d}(z) \rightarrow-\epsilon^{a b c d} \log |y-z|, \quad \theta^{a}(y) \bar{\theta}^{b}(z) \rightarrow \eta^{a b} \log |y-z|,
$$

it is easy to check that under the susy transformation generated by $v_{ \pm}^{a} q_{a}^{ \pm}+\bar{v}_{ \pm}^{a} \bar{q}_{a}^{ \pm}$, the worldsheet fields transform as

$$
\begin{gathered}
\delta \theta^{a}=v_{-}^{a}+v_{+}^{a} e^{\phi}-\frac{i}{2} \epsilon_{a b c d} \bar{v}_{+}^{b} x^{c d}, \\
\delta \bar{\theta}^{a}=\bar{v}_{-}^{a}+\bar{v}_{+}^{a} e^{\bar{\phi}}-\frac{i}{2} \epsilon_{a b c d} v_{+}^{b} x^{c d}, \\
\delta x^{a b}=i\left(v_{+}^{a} \theta^{b}-v_{+}^{b} \theta^{a}+\bar{v}_{+}^{a} \bar{\theta}^{b}-\bar{v}_{+}^{b} \bar{\theta}^{a}\right) \\
\delta \phi=\delta \bar{\phi}=0 .
\end{gathered}
$$

This has the following very important property: if we drop the ghosts $e^{\phi}$ and $e^{\bar{\phi}}$, then under supersymmetry, $\theta, \bar{\theta}$, and $x$ just transform under spacetime supersymmetry into functions of themselves. This means that spacetime supersymmetry acts (modulo ghost couplings) by an automorphism of the target space $M$ of the sigma model, and is a symmetry of the sigma model action (again modulo ghosts) if and only if the metric of $M$ is invariant under that automorphism. Thus, spacetime supersymmetry is just a supergroup of isometries of $M$. Knowledge of this fact will enable us to determine $M$ and the sigma model action in section 7 . Even if the $e^{\phi}$ and $e^{\bar{\phi}}$ terms are included, spacetime supersymmetry still acts by automorphisms of $M$, but now these are $\phi$ and $\bar{\phi}$-dependent automorphisms.

It is also illuminating to examine, in the present "almost flat" approximation to $\mathrm{AdS}_{3} \times$ $\mathbf{S}^{3}$, the structure of the spacetime supersymmetry currents. Recall that in flat Minkowski space, the susy currents for $q_{a}^{ \pm}$were holomorphic (i.e. $j_{\bar{z} a}^{ \pm}=0$ and $\bar{\partial} j_{z a}^{ \pm}=0$ ), while the susy currents for $\bar{q}_{a}^{ \pm}$were similarly anti-holomorphic, (i.e. $\bar{j}_{z a}^{ \pm}=0$ and $\partial \bar{j}_{\bar{z} a}^{ \pm}=0$ ). Is this true after the perturbation to $\mathrm{AdS}_{3} \times \mathbf{S}^{3}$ ? The susy transformations of (6.9) are symmetries of the quadratic part of the $\widehat{S}_{A d S}$. (They are not symmetries of the quartic terms as we have not determined the ... terms; also, as we will see in the next section, there are higher order corrections to the supersymmetry transformations. But the quadratic approximation to the Lagrangian is sufficient for our present purposes.) However, as symmetries of the free kinetic energy of $x, \theta, \bar{\theta}$, these transformations are not generated by purely holomorphic or antiholomorphic currents. Using the Noether method to find the currents that generate the symmetry (6.9) of the free action, one finds for example that $j_{\bar{z} a}^{+}$no longer vanishes 
in the almost flat approximation to AdS, but is equal to $j_{\bar{z} a}^{+}=\frac{i}{2} \epsilon_{a b c d}\left(\bar{\partial} \theta^{b}\right) x^{c d}$. There is thus a conserved supercurrent $\bar{\partial} j_{z a}^{+}+\partial j_{\bar{z} a}^{+}=0$, but it has both $(1,0)$ and $(0,1)$ pieces. In the flat case, the rotation symmetries have this property, but the supersymmetries are purely holomorphic or purely antiholomorphic. After deformation to $\operatorname{AdS}_{3} \times \mathbf{S}^{3}$ (with RR background), the spacetime supersymmetries are carried by mixtures of left and rightmovers.

\subsection{Structure Of Unbroken Supersymmetries}

The following further remark will help in determining the sigma model action $\widehat{S}_{A d S}$ which describes the system modulo ghost terms. Though there will be corrections to (6.9) of higher order in the fields, these formulas suffice for determining which supersymmetries are broken and which are unbroken in the classical vacuum of the sigma model at $x=0$. Setting $e^{\phi}$ and $e^{\bar{\phi}}$ to zero, we see from (6.9) that the unbroken supersymmetries (which act trivially on $\theta, \bar{\theta}$ if $x=0$ ) are precisely those with $v_{-}^{a}=\bar{v}_{-}^{a}=0$. In particular, in the sigma model, half of the supersymmetries are broken and half are unbroken.

It is instructive to calculate the algebra generated by the unbroken supersymmetries. The anticommutator of two unbroken supersymmetries, if not zero, must of course be an unbroken bosonic symmetry. The unbroken bosonic symmetries are the $S O(4)$ rotations around $P$. A straightforward computation shows that the anticommutator of two unbroken supersymmetries is indeed the generator of an infinitesimal rotation of the $x$ 's and $\theta$ 's. The unbroken supersymmetries and rotations generate a supergroup that we will call $S U^{\prime}(2 \mid 2)$; its properties are described more fully in the next section. We can think of the unbroken symmetry group $H \cong S U^{\prime}(2 \mid 2)$ as a supergroup of "rotations" of $x, \theta, \bar{\theta}$. The broken bosonic symmetries are the translations $\delta x=$ constant. Similarly the broken supersymmetries (with $v_{-}^{a}, \bar{v}_{-}^{a}$ nonzero) are translations of $\theta, \bar{\theta}$. There is a spontaneously broken translation symmetry for every coordinate $x, \theta, \bar{\theta}$, so the target space $M$ of the sigma model is a homogeneous space for the spacetime supersymmetry. The coordinates transform in the adjoint representation of $S U^{\prime}(2 \mid 2)$ (this will become more obvious in section 7 when we describe this supergroup in detail), so the broken symmetries, that is the translation generators of $x, \theta, \bar{\theta}$, do likewise.

Even though our analysis has been based on knowing the action only to very low order in the perturbation parameter $f$, it is highly plausible that the identification of the target space as a homogeneous space is general. If so, we can determine what $M$ must be. In fact, the spacetime supersymmetry of Type IIB string theory on $\mathrm{AdS}_{3} \times \mathbf{S}^{3} \times \mathrm{K} 3$ 
is $S U^{\prime}(2 \mid 2)^{2}$, where $G=S U^{\prime}(2 \mid 2)$ is the supergroup encountered in the last paragraph. For $M$ to be a homogeneous space for $G \times G$, it must be of the form $(G \times G) / H$ for some subgroup $H$ of $G \times G$; to get the right dimension for $M, H$ must be of dimension $6 \mid 8$. $H$ is in fact the supergroup generated by the unbroken supersymmetries at any given classical vacuum, which we may as well take to be the vacuum at $P$. From the analysis in the last paragraph, $H$ is therefore isomorphic to $G$. Moreover, to get the unbroken supersymmetries to transform in the adjoint representation of $H, H$ must be a diagonal subgroup of $G \times G$. (This embedding of $H \cong G$ in $G \times G$ is practically unique even without the knowledge of how the broken symmetries transform.) If $H$ is such a diagonal subgroup, then $M=(G \times G) / H$ is just a copy of $G$, with $G \times G$ acting on $M=G$ by left and right multiplication.

This identification of $M$ can further be confirmed by the following simple consideration. Ignoring fermionic variables, the $\mathrm{AdS}_{3} \times \mathbf{S}^{3}$ manifold can be identified as the group manifold $S U(1,1) \times S U(2)$. This is the bosonic reduction of the $S U^{\prime}(2 \mid 2)$ manifold. 12 So on this grounds alone, the bosonic reduction of $H$ must be the bosonic reduction of a diagonal subgroup of $G \times G$.

What about the terms in $S_{A d S}^{\prime}$ with ghosts? They cannot be written in a manifestly $G \times G$-invariant way, but must be $G \times G$-invariant anyway, with a suitable action of $G \times G$. We postpone the analysis of how this comes about to section 8 .

\subsection{NS Perturbation And Wess-Zumino Term}

We will see in the next section that, considering only operators with two derivatives, the sigma model with target space $G$ can be generalized to include one more $G \times G$ invariant interaction, namely a Wess-Zumino term. In fact, usually, in a sigma model with target space a group manifold, conformal invariance can be achieved only if such a term is present with the right coefficient. In the next section, we will see that the sigma model with $S U^{\prime}(2 \mid 2)$ target has an exceptional property: it is conformally invariant for any value of the Wess-Zumino coefficient (including zero). Thus, omitting the Wess-Zumino term, we get a conformal field theory description of the $\mathrm{AdS}_{3} \times \mathbf{S}^{3}$ model with RR background. But the Wess-Zumino term should also have a physical interpretation (as it admits a deformation of the $N=4$ symmetry of the flat model described in section 4 ).

12 To be more precise, it is the bosonic reduction of the alternative real form $S U^{\prime}(1,1 \mid 2)$ of $S U^{\prime}(2 \mid 2)$. 
In fact, its interpretation is rather simple. So far in this section, we have focused on giving an expectation value to the $\mathrm{RR}$ three-form fields on $\mathrm{AdS}_{3} \times \mathbf{S}^{3}$. One can also turn on an NS three form field, with the same ansatz (6.1). (In the interpretation of the model in terms of strings on $\mathbf{R}^{6} \times \mathrm{K} 3$ or $\mathbf{R}^{6} \times \mathbf{T}^{4}$, this corresponds to having NS as well as Dirichlet fivebranes wrapped on $\mathrm{K} 3$ or $\mathbf{T}^{4}$.) To first order in $f$, this perturbation is made by adding a vertex operator $V_{H}^{N S}$ of the $N S B$-field. As shown in section 5 , that vertex operator contains no ghost couplings, so if one perturbs the flat model by the NS three-form only, matter and ghosts are decoupled as in the flat case, while any generic combination of the RR and NS fields gives matter-ghost couplings.

In fact, any generic linear combination of RR and NS perturbations leads to the general structure we have found: the action contains a term $\bar{p} p$ (which appears in the RR vertex operator) as a result of which $p$ and $\bar{p}$ can be integrated out, to give a second order sigma model action for the group $S U^{\prime}(2 \mid 2)$. The NS perturbation appears as a Wess-Zumino term in this action. We will describe the spacetime supersymmetric Wess-Zumino term in section 7 and see its relation to $V_{H}^{N S}$.

If instead of a generic combination of RR and NS fields, one makes the NS perturbation only, then one has two options:

(1) There is no $p \bar{p}$ term in the action, so it is natural to use a description with first order kinetic energy for fermions. This description will be given in section 10 and turns out to be rather simple because the fermions can be treated as free fields.

(2) On the other hand, one can regard the NS model as a model obtained with a perturbation by $V_{H}^{N S}+\epsilon V_{H}^{R R}$, in the limit as $\epsilon \rightarrow 0$. For every nonzero $\epsilon$, there is a $\bar{p} p$ term, making it possible to integrate out $p$. Moreover, after rescaling the $\theta$ 's by a factor of $\epsilon^{-1 / 2}$, the resulting Lagrangian has a limit as $\epsilon \rightarrow 0$. This gives a different description of the NS model, as a WZW model of $S U^{\prime}(2 \mid 2)$.

It will be shown in section 10 that these two descriptions are equivalent by rewriting the currents of WZW $S U^{\prime}(2 \mid 2)$ model in terms of free fermions and an additional bosonic WZW model on $S U(2) \times S L(2)$. Whether one follows route (1) or route (2), the model with the NS perturbation only has left and right-moving $S U^{\prime}(2 \mid 2)$ current algebra, while any model that includes also (or only) an RR perturbation has spacetime supersymmetries that are carried by a mixture of left-movers and right-movers. 


\section{Sigma Model Of $S U^{\prime}(2 \mid 2)$}

\subsection{Construction Of The Model}

In this section, we will investigate the model that was suggested by the discussion in section 6 , namely the two-dimensional sigma model with target the group manifold of $G=S U^{\prime}(2 \mid 2), 13$

First of all, in general the group (or rather supergroup) $U(n \mid m)$ is the group of unitary transformations of a complex vector space of dimension $n \mid m$, that is, bosonic dimension $n$ and fermionic dimension $m$. An element $X$ of $U(n \mid m)$ can be represented by a matrix

$$
X=\left(\begin{array}{cc}
A & B \\
C & D
\end{array}\right),
$$

where $A$ and $D$ are $n \times n$ and $m \times m$ bosonic matrices, and $B$ and $C$ are $n \times m$ and $m \times n$ fermionic matrices. Such a matrix has a Berezinian or superdeterminant, characterized by the fact that for $X, Y \in U(n \mid m)$, Sdet $X Y=\operatorname{Sdet} Y X$ and that if $B=C=0$, $\operatorname{Sdet} X=\operatorname{det} A \operatorname{det}^{-1} B$.

The Lie algebra (or to be more precise, superalgebra) of $U(n \mid m)$ consists of matrices of the form

$$
x=\left(\begin{array}{ll}
a & b \\
c & d
\end{array}\right),
$$

where $a$ and $d$ are bosonic and $b$ and $c$ are fermionic; $a$ and $d$ are hermitian and $b$ and $c$ are hermitian conjugates. One defines the supertrace of $x$ as $\operatorname{Str} x=\operatorname{Tr} a-\operatorname{Tr} d$.

There are two similar ways to relate $U(n \mid m)$ to a group of dimension one less. We can require that $\operatorname{Str} x=0$ or equivalently that $\operatorname{Sdet} X=1$. This gives a supergroup $S U(n \mid m)$. Or we can take the quotient by scalars, considering $x$ trivial if it is a multiple of the identity and identifying two $X$ 's that are scalar multiples of each other.

For $n \neq m$, the two operations are equivalent to each other locally. If $x$ is a nonzero multiple of the identity, its supertrace is nonzero if $n \neq m$, so requiring the supertrace to be zero is equivalent at the Lie algebra level to removing the constants.

For $n=m$, the story is different. One has $\operatorname{Str} 1=0$, so asking that the supertrace of $x$ vanishes does not remove the scalars. One can form a smaller supergroup, of dimension two less than that of $U(n \mid n)$, by requiring that $\operatorname{Str} x=0$ and also working modulo the

13 Aspects of sigma models on supergroup manifolds have also been recently considered independently in 20]. 
constants. We will call this group $S U^{\prime}(n \mid n)$ (mathematically the name $\mathcal{A}_{n-1, n-1}$ has been used). The Lie algebra of $S U^{\prime}(n \mid n)$ consists of matrices as in (7.2) with $\operatorname{Tr} a=\operatorname{Tr} d=0$.

The bosonic part of $S U^{\prime}(n \mid n)$ is $S U(n) \times S U(n)$, generated by $a$ and $d$. The fermionic generators of $S U^{\prime}(n \mid n)$ - that is, the matrices $b$ and $c-\operatorname{transform}$ as $\mathbf{n} \otimes \overline{\mathbf{n}} \oplus \overline{\mathbf{n}} \otimes \mathbf{n}$. At this stage we notice a further coincidence that arises only for $n=2$. In this case, the representations $\mathbf{n}$ and $\overline{\mathbf{n}}$ are isomorphic, so the odd generators of $S U^{\prime}(2 \mid 2)$ consist of two copies of $\mathbf{n} \otimes \mathbf{n}$. As a result, it turns out that the group $S U^{\prime}(2 \mid 2)$ has a group $R=S L(2, \mathbf{R})$ of outer automorphisms, commuting with the bosonic generators and rotating the two fermionic copies of $\mathbf{2} \otimes \mathbf{2} .4$

To describe explicitly the Lie algebra of $S U^{\prime}(2 \mid 2)$ with its outer automorphism group, we proceed as follows. The bosonic part of $S U^{\prime}(2 \mid 2)$ is $S U(2) \times S U(2)$, which we identify at the Lie algebra level with $S O(4)$. The $\mathbf{2} \otimes \mathbf{2}$ of $S U(2) \times S U(2)$ is the vector of $S O(4)$. So the odd or fermionic generators of $S U^{\prime}(2 \mid 2)$ consist of a pair of $S O(4)$ vectors; we write them as $S_{a \alpha}$, where $a=1, \ldots, 4$ is a vector index of $S O(4)$, and $\alpha=1,2$ labels the two vectors. The outer automorphism group will act on the $\alpha$ index. The bosonic generators of $S U^{\prime}(2 \mid 2)$ transform in the adjoint or antisymmetric tensor representation of $S O(4)$; we write them as $K_{a b}$, where $a, b=1, \ldots, 4$ and $K_{a b}=-K_{b a}$. The Lie algebra of $S U^{\prime}(2 \mid 2)$ can be described by the following formulas:

$$
\begin{aligned}
{\left[K_{a b}, K_{c d}\right] } & =\delta_{a c} K_{b d}-\delta_{a d} K_{b c}-\delta_{b c} K_{a d}+\delta_{b d} K_{a c} \\
{\left[K_{a b}, S_{c \alpha}\right] } & =\delta_{a c} S_{b \alpha}-\delta_{b c} S_{a \alpha} \\
\left\{S_{a \alpha}, S_{b \beta}\right\} & =\frac{1}{2} \epsilon_{\alpha \beta} \epsilon_{a b c d} K^{c d} .
\end{aligned}
$$

Here $\epsilon_{\alpha \beta}$ and $\epsilon_{a b c d}$ are the antisymmetric tensors of $S L(2, \mathbf{R})$ and $S O(4)$ and we shall define $\epsilon_{12}=\epsilon^{21}=\epsilon_{1234}=1$. The Jacobi identity is readily verified; the $S L(2, \mathbf{R})$ invariance is manifest.

Now we would like to describe a two-dimensional sigma model, with target space the group manifold of $G=S U^{\prime}(2 \mid 2)$, which is invariant under the left and right action of $G$ on itself. The basic field of the sigma model will be a field $g$ that takes values in $G$; the model should be invariant under $G \times G$ acting by

$$
g \rightarrow A g B^{-1}, \text { with } A, B \in G
$$

14 After constructing the action, we will make a Wick rotation to a group $S U^{\prime}(1,1 \mid 2)$ that is more directly relevant to the physics in Lorentz signature. For this group, the outer automorphism group is $S U(2)$ rather than $S L(2, \mathbf{R})$. We will also consider later the case of Euclidean signature. 
By a sigma model, we mean a model with an action of the general form

$$
S=\frac{1}{2} \int d^{2} \sigma g_{I J} \partial_{i} \Phi^{I} \partial^{i} \Phi^{J},
$$

where $\Phi^{I}$ are coordinates on the group manifold, and $g_{I J}$ is a metric on $G$. The condition for the sigma model to be left and right invariant is that the metric $g$ should be invariant. Given such a metric, its restriction to the identity element $1 \in G$ is certainly invariant under the subgroup of $G \times G$ that leaves the identity element fixed (this is the diagonal subgroup defined by $A=B$ ). Conversely, any metric at the identity that is invariant under the stabilizer of the identity can be transported over the whole manifold using the $G \times G$ symmetry to give an invariant metric on the whole group manifold.

The tangent space to $G$ at the identity is naturally isomorphic with the Lie algebra of $G$; the diagonal subgroup $A=B$ acts on the tangent space by conjugation. So sigma models of this form are classified by $G$-invariant inner products on the Lie algebra of $G$. If we denote the inner product of two elements $x, y$ of the Lie algebra as $\langle x, y\rangle$, then the condition of invariance is that for any $x, y, z$ one has

$$
\langle[x, y\}, z\rangle+(-1)^{x y}\langle y,[x, z\}\rangle=0 .
$$

(Here [, \} denotes the bracket in the Lie superalgebra; we will henceforth write just [ , ].) Up to a scalar multiple, the most general invariant inner product on the $S U^{\prime}(2 \mid 2)$ Lie algebra reads

$$
\begin{aligned}
\left\langle K_{a b}, K_{c d}\right\rangle & =\epsilon_{a b c d} \\
\left\langle S_{a \alpha}, S_{b \beta}\right\rangle & =\delta_{a b} \epsilon_{\alpha \beta},
\end{aligned}
$$

with other components vanishing.

Note that, unlike most familiar situations encountered in physics, this inner product cannot conveniently be written as $\langle x, y\rangle=\operatorname{Tr} x y$ with the trace taken in some representation of $G$. Perhaps it is important to point out that the basic $2 \mid 2$-dimensional representation of $S U(2 \mid 2)$ cannot be interpreted as a representation of $S U^{\prime}(2 \mid 2)$ (operators that one would try to define as $S U^{\prime}(2 \mid 2)$ generators in this representation do not close on $S U^{\prime}(2 \mid 2)$ but on its extension $S U(2 \mid 2)$ ), so we cannot define a quadratic form on the $S U^{\prime}(2 \mid 2)$ Lie algebra via a supertrace in this representation. The smallest representation of $S U^{\prime}(2 \mid 2)$ is the adjoint representation. 
The sigma model action for a $G$-valued field $g$ can now be introduced. Up to a possible multiplicative constant, it is

$$
S=\frac{1}{2} \int d^{2} \sigma\left\langle g^{-1} \partial_{i} g, g^{-1} \partial_{i} g\right\rangle
$$

We would now like to expand this action near $g=1$, and compare the expansion to the action found from a different point of view in the last section. For this, we will pick an explicit parametrization of the group manifold and compute the action in detail.

\subsection{Evaluation Of The Action}

We wish to evaluate the action explicitly up to quartic order in the fields. Let us first discuss this procedure in general, for any Lie group $G$ with generators $T_{A}$ and an invariant

quadratic form $\langle$,$\rangle on the Lie algebra. We introduce coordinates \Phi^{A}$ and parametrize an element of the group near the identity as

$$
g=\exp \left(\Phi^{A} T_{A}\right)
$$

Now we compute

$$
g^{-1} d g=d \Phi^{A} T_{A}+\frac{1}{2} d \Phi^{A} \Phi^{B}\left[T_{A}, T_{B}\right]+\frac{1}{6} d \Phi^{A} \Phi^{B} \Phi^{C}\left[\left[T_{A}, T_{B}\right], T_{C}\right]+O\left(\Phi^{4}\right) .
$$

Hence we have

$$
\left\langle g^{-1} \partial_{i} g, g^{-1} \partial_{i} g\right\rangle=\partial_{i} \Phi^{A} \partial_{i} \Phi^{B}\left\langle T_{A}, T_{B}\right\rangle-\frac{1}{12} \partial_{i} \Phi^{A} \Phi^{B} \partial_{i} \Phi^{C} \Phi^{D}\left\langle\left[T_{A}, T_{B}\right],\left[T_{C}, T_{D}\right]\right\rangle+O\left(\Phi^{5}\right)
$$

One can also explicitly describe the transformation of the $\Phi^{A}$ under the $G \times G$ symmetry. We introduce infinitesimal parameters $\epsilon_{L}^{A}, \epsilon_{R}^{B}$ for the left and right group actions, and abbreviate $\Phi=\Phi^{A} T_{A}, \epsilon_{L}=\epsilon_{L}^{A} T_{A}, \epsilon_{R}=\epsilon_{R}^{A} T_{A}$. The transformation

$$
\exp (\Phi) \rightarrow \exp \left(\epsilon_{L}\right) \exp (\Phi) \exp \left(-\epsilon_{R}\right)
$$

can be expanded in powers of $\Phi$ to give

$$
\delta \Phi=\epsilon_{L}-\epsilon_{R}+\frac{1}{2}\left[\epsilon_{L}+\epsilon_{R}, \Phi\right]+\frac{1}{12}\left[\left[\epsilon_{L}-\epsilon_{R}, \Phi\right], \Phi\right]+O\left(\Phi^{3}\right) .
$$


Clearly, the unbroken symmetry, which leaves fixed the classical vacuum at $g=1$, is generated by $\epsilon_{L}+\epsilon_{R}$, and the broken symmetry, which shifts the $g=1$ vacuum, is generated by $\epsilon_{L}-\epsilon_{R}$. The conserved currents generating the symmetries are

$$
\begin{aligned}
J_{L}\left(\epsilon_{L}\right)= & \left\langle\epsilon_{L}, d g g^{-1}\right\rangle=\left\langle\epsilon_{L}, d \Phi^{A} T_{A}-\frac{1}{2} d \Phi^{A} \Phi^{B}\left[T_{A}, T_{B}\right]\right. \\
& \left.+\frac{1}{6} d \Phi^{A} \Phi^{B} \Phi^{C}\left[\left[T_{A}, T_{B}\right], T_{C}\right]\right\rangle+O\left(\Phi^{4}\right), \\
J_{R}\left(\epsilon_{R}\right)= & -\left\langle\epsilon_{R}, g^{-1} d g\right\rangle=-\left\langle\epsilon_{R}, d \Phi^{A} T_{A}+\frac{1}{2} d \Phi^{A} \Phi^{B}\left[T_{A}, T_{B}\right]\right. \\
& \left.+\frac{1}{6} d \Phi^{A} \Phi^{B} \Phi^{C}\left[\left[T_{A}, T_{B}\right], T_{C}\right]\right\rangle+O\left(\Phi^{4}\right) .
\end{aligned}
$$

For the $S U^{\prime}(2 \mid 2)$ case, we further write

$$
\Phi=\frac{1}{4} \epsilon_{a b c d} x^{a b} K^{c d}+\theta^{a \alpha} S_{a \alpha}
$$

with bosonic and fermionic coordinates $x^{a b}, \theta^{a \alpha}$. (The coefficient of $K^{c d}$ has been written as a multiple of $\epsilon_{a b c d} x^{a b}$ rather than $x^{c d}$, since this leads to formulas that agree better with the $S U(4)$-invariant formulas of the flat case.) In this case we can compute

$$
d \Phi^{A} \Phi^{B}\left[T_{A}, T_{B}\right]=\left(d x^{a e} x^{b e}-\frac{1}{2} \epsilon^{a b c d} \epsilon_{\alpha \beta} \theta^{c \alpha} \theta^{d \beta}\right) K_{a b}+\frac{1}{2} \epsilon_{a b c d}\left(d x^{a b} \theta^{c \alpha}-x^{a b} d \theta^{c \alpha}\right) S_{\alpha}^{d}
$$

With the aid of this formula, one finds that to this order, the action is

$$
\begin{aligned}
S= & \frac{1}{2} \int d^{2} \sigma\left(\frac{1}{4} \epsilon_{a b c d} d x^{a b} * d x^{c d}-d \theta^{a \alpha} * d \theta^{a \beta} \epsilon_{\alpha \beta}\right. \\
& -\frac{1}{12} \epsilon_{a b a^{\prime} b^{\prime}}\left(d x^{a e} x^{b e}-\frac{1}{2} \epsilon_{a b c d} d \theta^{c \alpha} \theta^{d \beta} \epsilon_{\alpha \beta}\right) *\left(d x^{a^{\prime} e^{\prime}} x^{b^{\prime} e^{\prime}}-\frac{1}{2} \epsilon_{a^{\prime} b^{\prime} c^{\prime} d^{\prime}} d \theta^{c^{\prime} \alpha} \theta^{d^{\prime} \beta} \epsilon_{\alpha \beta}\right) \\
& \left.+\frac{1}{48} \epsilon_{\alpha \beta} \epsilon_{a b c d}\left(d x^{a b} \theta^{c \alpha}-x^{a b} d \theta^{c \alpha}\right) * \epsilon_{a^{\prime} b^{\prime} c^{\prime} d}\left(d x^{a^{\prime} b^{\prime}} \theta^{c^{\prime} \beta}-x^{a^{\prime} b^{\prime}} d \theta^{c^{\prime} \beta}\right)\right) .
\end{aligned}
$$

Here to avoid clutter, we have adopted the convention that if $A$ and $B$ are fields, $d A * d B$ is short for $\partial_{i} A \partial^{i} B$. Using (7.14), we can also now compute that the supersymmetry currents are

$$
\begin{aligned}
& S_{L \alpha}^{a}=d \theta_{\alpha}^{a}-\frac{1}{4} \epsilon^{a b c d}\left(d \theta_{\alpha}^{b} x^{c d}-\theta_{\alpha}^{b} d x^{c d}\right) \\
& S_{R \alpha}^{a}=-d \theta_{\alpha}^{a}-\frac{1}{4} \epsilon^{a b c d}\left(d \theta_{\alpha}^{b} x^{c d}-\theta_{\alpha}^{b} d x^{c d}\right)
\end{aligned}
$$


up to second order in the fields. The rotation currents $K_{L a b}$ (generating the left action of $\left.K_{a b}\right)$ can be similarly computed:

$$
K_{L}^{a b}=d x^{a b}-\frac{1}{2} \epsilon^{a b c d} d x^{c e} x^{d e}+\frac{1}{2} \epsilon_{\alpha \beta}\left(d \theta^{a \alpha} \theta^{b \beta}-d \theta^{b \alpha} \theta^{a \beta}\right) .
$$

When it is likely to cause no confusion, we use the same name $K$ or $S$ for an element of the Lie algebra and the corresponding conserved current or charge.

To analyze the ghost couplings in the next section, we will also need the MaurerCartan equations. For a general group $G$ with Lie algebra basis $T_{A}$, obeying $\left[T_{A}, T_{B}\right]=$ $f_{A B}^{C} T_{C}$, define the right-invariant currents by expanding $d g g^{-1}=\sum_{A} T_{A} J^{A}$. The $J^{A}$ are related to $J_{A}=\left\langle T_{A}, d g g^{-1}\right\rangle$ by "raising an index" with the quadratic form $\langle$,$\rangle . Since$ $d\left(d g g^{-1}\right)=-d g g^{-1} \wedge d g g^{-1}$, the $J^{A}$ obey the Maurer-Cartan equations

$$
d J^{A}=-\frac{1}{2} f_{B C}^{A} J^{B} \wedge J^{C} .
$$

Of course, the $J_{A}$ obey an equivalent equation. In the case of $S U^{\prime}(2 \mid 2)$, these equations give

$$
\begin{aligned}
d S_{a \alpha} & =-\frac{1}{2} \epsilon_{a b c d} K^{b c} \wedge S_{\alpha}^{d} \\
d K^{a b} & =\frac{1}{2} \epsilon^{a b c d} K^{c e} \wedge K^{d e}-\epsilon_{\alpha \beta} S^{a \alpha} \wedge S^{b \beta},
\end{aligned}
$$

where here the currents can be either left or right currents.

Finally, we shall meet in the next section the right-invariant two-forms

$$
\omega_{L}^{\alpha \beta}=S_{L}^{a \alpha} \wedge S_{L}^{a \beta}
$$

Note that $\omega_{L}^{\alpha \beta}=\omega_{L}^{\beta \alpha}$; the $\omega_{L}$ 's transform in the spin one representation of the $S U(2)$ group $R$ of outer automorphisms. A small calculation using the Maurer-Cartan equations shows that the $\omega_{L}$ 's viewed as two-forms on the group manifold are closed, $d \omega_{L}^{\alpha \beta}=\frac{1}{2} \epsilon_{a b c d} S^{a \alpha} \wedge$ $S^{b \beta} \wedge K^{c d}+\alpha \leftrightarrow \beta=0$, where fermi statistics have been used. Since the second Betti number of the $S U^{\prime}(2 \mid 2)$ group manifold is zero, the two-forms $\omega_{L}^{\alpha \beta}$ are actually exact, $\omega_{L}^{\alpha \beta}=d \lambda_{L}^{\alpha \beta}$ for some $\lambda_{L}^{\alpha \beta}$. Though the $\omega_{L}$ 's are right-invariant, the $\lambda_{L}$ 's cannot be chosen to be right-invariant. The $\omega_{L}$ 's are thus non-trivial if viewed as elements of the Lie algebra cohomology of $S U^{\prime}(2 \mid 2)$ and are, in fact, related to the existence of nontrivial central extensions of this group. But the fact that $\omega_{L}^{\alpha \beta}=d \lambda_{L}^{\alpha \beta}$ for some $\lambda_{L}$ 's, even though not right-invariant, means that the interaction terms

$$
\int \sum_{\alpha \beta} d_{\alpha \beta} \omega_{L}^{\alpha \beta}
$$


that one might think of adding to a two-dimensional sigma model action (with "coupling constants" $\left.d_{\alpha \beta}\right)$ are actually trivial. This fact will be important.

It will also be useful to note that $\omega_{L}^{\alpha \beta}$ is invariant under all $S U^{\prime}(2 \mid 2) \times S U^{\prime}(2 \mid 2)$ transformations except for the left susy transformations generated by the charges $\oint S_{L}^{a \alpha}$. The commutation relations of (7.3) together with the Maurer-Cartan equations of (7.21) imply that

$$
\left[\omega_{L}^{\alpha \beta}, \oint S_{L}^{a \gamma}\right]=\frac{1}{2} \epsilon_{a b c d}\left(\epsilon^{\beta \gamma} K_{L}^{b c} S_{L}^{d \alpha}+\epsilon^{\alpha \gamma} K_{L}^{b c} S_{L}^{d \beta}\right)=-\epsilon^{\beta \gamma} d S_{L}^{a \alpha}-\epsilon^{\alpha \gamma} d S_{L}^{a \beta} .
$$

Since $\left.\omega_{L}^{\alpha \beta}=d \lambda_{L}^{\alpha \beta}, 7.24\right)$ implies that

$$
\left[\lambda_{L}^{\alpha \beta}, \oint S_{L}^{a \gamma}\right]=-\epsilon^{\beta \gamma} S_{L}^{a \alpha}-\epsilon^{\alpha \gamma} S_{L}^{a \beta}+d M_{L}^{a \alpha \beta \gamma}
$$

for some $M_{L}^{a \alpha \beta \gamma}$. Similarly, one can show that

$$
\left[\omega_{R}^{\alpha \beta}, \oint S_{R}^{a \gamma}\right]=-\epsilon^{\beta \gamma} d S_{R}^{a \alpha}-\epsilon^{\alpha \gamma} d S_{R}^{a \beta}, \quad\left[\lambda_{R}^{\alpha \beta}, \oint S_{R}^{a \gamma}\right]=-\epsilon^{\beta \gamma} S_{R}^{a \alpha}-\epsilon^{\alpha \gamma} S_{R}^{a \beta}+d M_{R}^{a \alpha \beta \gamma}
$$

where $\omega_{R}^{\alpha \beta}=S_{R}^{a \alpha} \wedge S_{R}^{a \beta}=d \lambda_{R}^{\alpha \beta}$.

\subsection{The Signature Of Spacetime}

Because of the $\epsilon_{a b c d}$ symbol multiplying the term quadratic in $x$, the action introduced above describes motion of strings in a target space with signature ---+++ . The reason for this signature is that we started with the group $S U^{\prime}(2 \mid 2)$, whose bosonic part is $S U(2) \times S U(2)$. Going back to the description of the Lie algebra in eqn. (7.2), if $a$ and $d$ are generators of the two $S U(2)$ 's, then our metric on the Lie algebra was a multiple of $\langle a, a\rangle=-\operatorname{Tr} a^{2},\langle d, d\rangle=\operatorname{Tr} d^{2}$. Thus one $S U(2)$ has --- signature, and the other has +++ signature. To get Lorentz signature, we should replace one of the $S U(2)$ 's by $S U(1,1)$ (which is locally isomorphic to $S L(2, \mathbf{R})$ ). $S U(1,1) \times S U(2)$ is the bosonic part of a supergroup that we might call $S U^{\prime}(1,1 \mid 2)$. Since the analytic continuation from $S U(2)$ to $S U(1,1)$ maps the quadratic form - Tr $a^{2}$ of signature - - - to a form of signature -++ , the $S U^{\prime}(1,1 \mid 2)$ model has Lorentz signature -+++++ . Indeed, the manifold $\mathrm{AdS}_{3} \times \mathbf{S}^{3}$ (with Lorentz signature on $\mathrm{AdS}_{3}$ ) can be understood as the group manifold $S U(1,1) \times S U(2)$.

With Euclidean signature, $\mathrm{AdS}_{3}$ is not a group manifold, so there is no real form of $S U^{\prime}(2 \mid 2)$ that will give a positive signature $\mathrm{AdS}_{3} \times \mathbf{S}^{3}$ model. However, at the cost of losing 
the reality of the Lagrangian, it is possible, as follows, to make an analytic continuation to a model with Euclidean signature in the target space. Let $x^{+}$and $x^{-}$be the self-dual and anti-self-dual parts of $x$. Then the analytic continuation $x^{+} \rightarrow i x^{+}$(with no change in $x^{-}$) maps to a section in which the bosonic part of the action is real and positive definite. However, there is apparently no change of variables that makes the resulting couplings to fermions real. This should not be a complete surprise. Irrespective of our formalism, the Euclidean version of $\mathrm{AdS}_{3} \times \mathbf{S}^{3}$ cannot be represented with real fields. For example, the threeform field $H$ that enters the $\mathrm{AdS}_{3} \times \mathbf{S}^{3}$ supergravity solution obeys a self-duality condition which with Euclidean signature contains a factor of $i$, which appeared in subsection (6.1).

\subsection{Adding A Wess-Zumino Term}

To generalize the sigma model while preserving its symmetries - including twodimensional conformal invariance - the remaining option is to add a term

$$
\int B_{I J} d \Phi^{I} \wedge d \Phi^{J}
$$

with $B$ a two-form on the $S U^{\prime}(2 \mid 2)$ manifold. (We already analyzed in (7.23) some examples of such terms that happen to be trivial.) There are in fact no $S U^{\prime}(2 \mid 2) \times S U^{\prime}(2 \mid 2)$-invariant two-forms on the group manifold. However, for invariance of the interaction (7.27), it suffices that the three-form $H=d B$ should be invariant. $H$ is of course automatically closed. Conversely, given a left and right-invariant and closed three-form $H$, one can construct a new invariant interaction - the Wess-Zumino term - that is defined locally as $(7.27)$.

A three-form $H$ on the $S U^{\prime}(2 \mid 2)$ manifold with the necessary properties is determined by a third order antisymmetric function on the Lie algebra. Up to a scalar multiple, there is a unique such function. It can be defined by

$$
\Psi(x, y, z)=\langle x,[y, z]\rangle
$$

The resulting interaction can be written most invariantly as

$$
S^{\prime}=\int_{X} d^{3} y \epsilon^{i j k}\left\langle g^{-1} \partial_{i} g,\left[g^{-1} \partial_{j} g, g^{-1} \partial_{k} g\right]\right\rangle .
$$

The integration is carried out over a three-manifold $X$ whose boundary is spacetime. 
Up to third order in the fields, the integrand is simply

$$
\epsilon^{i j k}\left\langle\partial_{i} \Phi,\left[\partial_{j} \Phi, \partial_{k} \Phi\right]\right\rangle=\partial_{i}\left(\epsilon^{i j k}\left\langle\Phi,\left[\partial_{j} \Phi, \partial_{k} \Phi\right]\right\rangle\right) .
$$

An integration by parts thus reduces the Wess-Zumino action in cubic order to an ordinary integral over spacetime:

$$
S^{\prime}=\int d^{2} \sigma \epsilon^{i j}\left\langle\Phi,\left[\partial_{i} \Phi, \partial_{j} \Phi\right]\right\rangle .
$$

For $G=S U^{\prime}(2 \mid 2)$, we get, to this order,

$$
S^{\prime}=\int d^{2} \sigma \epsilon^{i j}\left(\partial_{i} x^{a b} \partial_{j} x^{c b} x_{a c}-\frac{3}{2} \epsilon_{a b c d} x^{c d} \epsilon_{\alpha \beta} \partial_{i} \theta^{a \alpha} \partial_{j} \theta^{b \beta}\right) .
$$

But (7.32) describes the first order deformation of flat $\mathbf{R}^{6}$ by an $H$ field from the NS sector; it is in fact the integrated vertex operator of section 5 for an antisymmetric NS-NS field with $B_{m n}=\left(\sigma_{m}\right)^{a b}\left(\sigma_{n}\right)^{c b} x^{a c}$. Note that this $B$ field satisfies $\partial^{m} B_{m n}=\partial_{p} \partial_{p} B_{m n}=0$, so its vertex operator can be evaluated using the methods of section 5 .

The general $G \times G$-invariant invariant action that is conformally invariant at the classical level is

$$
I=\frac{S}{f^{2}}+i k S^{\prime}
$$

$f$ is a constant that one might interpret as the inverse radius of $\mathrm{AdS}_{3} \times \mathbf{S}^{3} ; k$ determines the "level" of the Wess-Zumino coupling.

\subsection{Interpretation Of Parameters}

In the application to string theory, $\mathrm{AdS}_{3} \times \mathbf{S}^{3}$ arises by compactification on $\mathbf{T}^{4}$ or K3 with onebranes and fivebranes. In general, let $Q_{5}^{N S}$ and $Q_{5}^{R R}$ be the numbers of NS and RR fivebranes, and let $Q_{1}^{N S}$ and $Q_{3}^{R R}$ be the analogous onebrane numbers.

One parameter is easy to identify: $k$ (if topologically normalized by including a constant that we have omitted) simply equals $Q_{5}^{N S}$. In fact, NS fivebranes determine, in the usual way, an NS $H$-field with a topologically quantized flux. Elementary strings are electric-magnetic dual to NS fivebranes, and $Q_{5}^{N S}$ determines the "level" of the WessZumino interaction. ( $Q_{5}^{R R}$ would similarly determine the level of a Wess-Zumino interaction for a $D$-string probe, but in the present paper we are considering elementary string actions only.)

On the other hand, $f^{-1}$ is the radius of the $\mathrm{AdS}_{3} \times \mathbf{S}^{3}$ spacetime. This radius, when it is large, can be measured by probing $\mathrm{AdS}_{3} \times \mathbf{S}^{3}$ by massless particles. The long distance 
action for massless particles is governed by supergravity, so - when the radius is large - it can be determined in terms of the brane charges by solving supergravity equations. In the special cases $Q_{5}^{N S}=0$ and $Q_{5}^{R R}=0$, the solution is given in [9]. In general, the solution of the supergravity equations gives

$$
\frac{1}{f^{2}}=\sqrt{\left(Q_{5}^{N S}\right)^{2}+\lambda^{2}\left(Q_{5}^{R R}\right)^{2}}
$$

with $\lambda$ the ten-dimensional string coupling constant. When $Q_{5}^{R R}=0$, this relation becomes $f^{-2}=|k|$. This is the familiar relation between metric and $H$-field on the group manifold which determines the model to be a WZW model of $S U^{\prime}(2 \mid 2)$. It thus has left and right-moving $S U^{\prime}(2 \mid 2)$ current algebra, and will be studied from that point of view in section 10. In general, if the charges are real, one has $1 / f^{2} \geq|k|$.

Supersymmetry requires that the onebrane charges $\left(Q_{1}^{N S}, Q_{1}^{R R}\right)$ be a multiple of the fivebrane charges $\left(Q_{5}^{N S}, Q_{5}^{R R}\right)$. So we can write

$$
\left(Q_{5}^{N S}, Q_{5}^{R R}\right)=Q_{5}(p, q), \quad\left(Q_{1}^{N S}, Q_{1}^{R R}\right)=Q_{1}(p, q),
$$

with relatively prime integers $p, q$ and integers $Q_{1}, Q_{5}$. The parameter $Q_{1}$ does not enter the $S U^{\prime}(2 \mid 2)$ sigma model, but it determines a certain relation between the string coupling constant and the volume $v$ of the $\mathrm{K} 3$ or $\mathbf{T}^{4}$. In fact, $v$ is determined by equating the tensions of the strings derived from onebranes and fivebranes 15 , via $v T_{5} Q_{5}=T_{1} Q_{1}$. With $T_{1}=\sqrt{p^{2}+q^{2} / \lambda^{2}}, T_{5}=\sqrt{\left(p / \lambda^{2}\right)^{2}+q^{2} / \lambda^{2}}$, we get

$$
v=\frac{Q_{1} \lambda}{Q_{5}} \sqrt{\frac{p^{2} \lambda^{2}+q^{2}}{p^{2}+\lambda^{2} q^{2}}} .
$$

Our treatment in the present paper is valid in the limit $\lambda \rightarrow 0$, with $\lambda q, p, Q_{1}$, and $Q_{5}$ fixed. In this limit (assuming $\lambda q \neq 0$ ), the formula simplifies to

$$
v=\frac{Q_{1}}{Q_{5}} \frac{\lambda q}{\sqrt{p^{2}+(\lambda q)^{2}}} .
$$

As $p, q, Q_{1}$, and $Q_{5}$ are integers, $v$ is not a continuous parameter for given $\lambda$, but with $\lambda$ very small and $q \sim 1 / \lambda$, the possible values of $v$ are very closely spaced. In our perturbative treatment, $v$ appears to be a continuous parameter. The fundamental reason for this is that, as we are not considering $D$-brane probes in the present paper, but only fundamental strings, we do not see the quantization of RR flux and hence $Q_{5}^{R R}$ and $Q_{1}^{R R}$ appear to be real-valued parameters. Quantization of RR flux is - as always - a nonperturbative phenomenon from the point of view of weakly coupled perturbative string theory.

15 This condition is equivalent to minimizing the tension in the Einstein frame of the bound string made of wrapped fivebranes and onebranes. 


\subsection{Conformal Field Theory}

The sigma model Lagrangian (7.33) can be constructed for any Lie group or supergroup $G$ with invariant quadratic form $\langle$,$\rangle . Generically, this Lagrangian has a nonzero$ beta function unless $f$ and $k$ are suitably related. However, we will now argue that for $S U^{\prime}(2 \mid 2)$, the beta function vanishes for any $f, k$. This means that in this particular case, we have a conformal field theory description of a Ramond-Ramond background in string theory.

The first step is to look at the one-loop beta function. For any $G$, it is proportional to the quadratic form on the Lie algebra that is defined by

$$
\operatorname{Str}_{a d j} x y
$$

where $\operatorname{Str}_{a d j}$ is the supertrace in the adjoint representation. For conventional simple Lie groups, one defines an invariant $c_{2}(G)$ by stating that (7.37) equals $c_{2}(G)$ times a basic quadratic form $\langle$,$\rangle . For S U^{\prime}(2 \mid 2)$, or in general $S U^{\prime}(n \mid n)$, (7.37) vanishes, and hence the one-loop beta function is zero. This may be shown by explicit computation. Since any invariant quadratic form on the Lie algebra is a multiple of $\langle$,$\rangle , it is sufficient to consider$ the case that

$$
x=y=\left(\begin{array}{cc}
1 & 0 \\
0 & -1
\end{array}\right)
$$

equal diagonal generators of one of the $S U(2)$ 's in $S U^{\prime}(2 \mid 2)$. Under this generator, the bosonic part of the adjoint representation has eigenvalues $2,-2,0,0,0,0$, while the fermionic part has eight states of eigenvalue \pm 1 . So the vanishing comes from $2^{2}+(-2)^{2}-8 \cdot 1^{2}=0$. A more conceptual proof can be given by comparing to $U(n \mid n)$. We will omit this argument (which is similar in spirit to arguments we give below for the all orders beta function).

We will give two arguments to show that the $S U^{\prime}(n \mid n)$ beta function vanishes to all orders; one of the arguments shows that the beta function vanishes exactly and not just to all orders of perturbation theory. Since one of the arguments is based on showing that the $S U^{\prime}(n \mid n)$ beta function is independent of $n$, we pause here to show that this beta function is zero for $S U^{\prime}(1 \mid 1)$. Indeed, $S U^{\prime}(1 \mid 1)$ has no bosonic generators at all and is an abelian supergroup, so the $S U^{\prime}(1 \mid 1)$ sigma model is a free conformal field theory. 
We will show that the beta function of the $S U^{\prime}(n \mid n)$ sigma model vanishes by comparing it to properties of a $U(n \mid n)$ sigma model. In $U(n \mid n)$, one can define an invariant quadratic form by a supertrace in the $n \mid n$-dimensional fundamental representation:

$$
\langle x, y\rangle=\operatorname{Str} x y .
$$

There is also an invariant but degenerate quadratic form $\operatorname{Str} x \cdot \operatorname{Str} y$. The sigma model action thus has two possible couplings

$$
S_{U(n \mid n)}=\int d^{2} \sigma\left(\frac{1}{2 f^{2}} \operatorname{Str}\left(g^{-1} d g\right)^{2}+h\left(\operatorname{Str} g^{-1} d g\right)^{2}\right),
$$

with constants $f, h$. One can also have a Wess-Zumino coupling, which we do not write explicitly. Its inclusion does not affect the argument.

It is useful to single out two generators of the Lie algebra,

$$
I=\left(\begin{array}{ll}
1 & 0 \\
0 & 1
\end{array}\right), \quad L=\left(\begin{array}{cc}
1 & 0 \\
0 & -1
\end{array}\right)
$$

Let $T_{a}$ be a basis of generators that are orthogonal to both $I$ and $L$. In the basis $I, L$, and $T_{a}$, the nonzero brackets of $U(n \mid n)$ take the form

$$
\begin{aligned}
{\left[L, T_{a}\right] } & =c_{a b} T_{b} \\
{\left[T_{a}, T_{b}\right] } & =f_{a b}^{c} T_{c}+d_{a b} I .
\end{aligned}
$$

Here $f_{a b}^{c}$ are the structure constants of $S U^{\prime}(n \mid n)$, and $c_{a b}, d_{a b}$ are constants, whose details will not concern us, that reflect the fact that $L$ generates an outer automorphism of $S U^{\prime}(n \mid n)$ and $I$ appears as a central extension.

Because of this structure of the Lie algebra, the Lagrangian takes a very simple form. We parametrize the group manifold by

$$
g=\exp \left(u I+v L+\Phi^{a} T_{a}\right)
$$

The quantities $g^{-1} d g$ via which the Lagrangian is expressed can be expanded in terms of repeated commutators (the first few terms are in (7.10) ). $L$ never appears on the right hand side except in the linear term $d \Phi$, because it never appears on the right hand side of the commutation relations (7.42). Likewise, since all commutators $[I, \cdot]$ vanish, $u$ does not appear in $g^{-1} d g$ except in the linear term. Because of these facts, $u$ only appears in the 
action in a quadratic $d u d v$ term. The terms that do not involve $I$ or $L$ at all just give the $S U^{\prime}(n \mid n)$ sigma model action. So we get the simple structure:

$$
S_{U(n \mid n)}=S_{S U^{\prime}(n \mid n)}+\int d^{2} \sigma\left(\frac{2 n d u d v}{f^{2}}+n^{2} h(d v)^{2}+\frac{1}{f^{2}} \Delta L\left(v, \Phi^{a}\right)\right) .
$$

Because the kinetic energy is of the form $d u d v+d v^{2}$, there is a $\langle u u\rangle$ and a $\langle u v\rangle$ propagator, but no $\langle v v\rangle$ propagator. The field $v$ is coupled to the $S U^{\prime}(n \mid n)$ fields $\Phi^{a}$ by couplings $\Delta L$, whose details need not concern us. The important thing is that there are no such couplings involving $u$.

Since only $v$ and not $u$ appears in the interaction vertices and the $\langle v v\rangle$ propagator vanishes, it follows that all $U(n \mid n)$ Feynman diagrams all of whose external lines are in $S U^{\prime}(n \mid n)$ are the same as $S U^{\prime}(n \mid n)$ Feynman diagrams. Hence the beta function of the $S U^{\prime}(n \mid n)$ theory is determined by the renormalization of the $\operatorname{Str}\left(g^{-1} d g\right)^{2}$ term for $U(n \mid n)$. But here are two reasons that this renormalization vanishes:

(1) The operator $\mathcal{O}=\operatorname{Str}\left(g^{-1} d g\right)^{2}$ contains a $d u d v$ term. But the $s$-loop effective action, for all $s \geq 1$, is a function of only $v$ and not $u$, since $u$ nowhere appears in the interaction vertices. So there can be no infinity proportional to $\mathcal{O}$. Note that this argument is not limited to perturbation theory.

(2) Consider the $n$-dependence of the $U(n \mid n)$ beta function. To probe it, we parametrize the group by $g=\exp \left(\sum_{A} \Phi^{A} T_{A}\right)$ where now $T_{A}$ runs over all generators of the Lie algebra and $\Phi^{A}$ is a set of coordinates on the group manifold. To probe the beta function, we look at the two point function $\langle\operatorname{Str} C \Phi(x) \operatorname{Str} D \Phi(y)\rangle$ with $c$-number-wave functions $C$ and $D$ taking values in the Lie algebra. The general form of the two point function allowed by $U(n \mid n)$ invariance is

$$
\langle\operatorname{Str} C \Phi(x) \operatorname{Str} D \Phi(y)\rangle=E(x-y) \operatorname{Str} C D+F(x-y) \operatorname{Str} C \operatorname{Str} D,
$$

with functions $E, F .16$ Now let us consider the $n$ dependence of this two point function. Feynman diagrams in $U(n \mid n)$ theory can be constructed using "t Hooft's "double line" notation for fields in the adjoint representation of a unitary group or supergroup [21]. The $n$-dependence comes entirely from supertraces that arise from an "index loop" in a

16 To avoid infrared divergences in this discussion, one could, for example, add to the Lagrangian a mass term $\operatorname{Str} g$ that is invariant under the diagonal subgroup of the $U(n \mid n) \times U(n \mid n)$ symmetry of the sigma model. The argument uses only invariance under this diagonal subgroup. 
Feynman diagram. For $U(n \mid m)$, the supertrace of the identity in the fundamental representation equals $n-m$, and one gets this factor for each index loop. For $U(n \mid n)$, this factor vanishes, and hence all diagrams that contain index loops vanish. Hence the two point function (7.45) is independent of $n$. So in particular $E$, which determines the $S U^{\prime}(n \mid n)$ beta function, is finite for all $n$ if it is finite for some $n$. We have already noted that the $S U^{\prime}(1 \mid 1)$ theory is free and finite, so the $E$ function in (7.45) must have no infinity for $n=1$ and hence for all $n$. Hence, the $S U^{\prime}(n \mid n)$ theory is finite for all $n$.

To avoid confusion, we should note that we are not claiming that in $U(n \mid n)$, there is no infinite renormalization of the $\left(\operatorname{Str} g^{-1} d g\right)^{2}$ term in the Lagrangian. On the contrary, for $U(n \mid n), \operatorname{Str}_{a d j} L^{2} \neq 0$, so there is such an infinity at one-loop order. (For $U(n \mid n)$, $\operatorname{Str}_{a d j} x y$ is a multiple of $\operatorname{Str} x \operatorname{Str} y$, so the one-loop infinity is a multiple of (Str $\left.g^{-1} d g\right)^{2}$.) Likewise, we make no claim that the $F$ term in the correlator is finite, only the $E$ term which is related to the $S U^{\prime}(n \mid n)$ beta function.

\section{Structure Of The Ghost Couplings}

\subsection{First Order Treatment}

In section 7 , we argued that the $\mathrm{AdS}_{3} \times \mathbf{S}^{3}$ model with Ramond-Ramond background can be described by a sigma model with couplings to ghost fields $e^{\phi}$ and $e^{\bar{\phi}}$. The simplifying feature that makes the theory manageable is that the ghost couplings involve only positive powers of $e^{\phi}$ and $e^{\bar{\phi}}$. Hence, there is a consistent truncation in which they are neglected in first approximation. We have described this truncation in the last section in terms of a sigma model with manifest spacetime supersymmetry. But looking back to the formulas of section 7 , it is clear that the ghost couplings do not have manifest supersymmetry. Instead, they have a much more complex structure that we will analyze here.

The ghost couplings that come directly from the vertex operators are as we saw in equation $(6.2)$,

$$
S_{\phi}=\frac{i}{8} \int d^{2} \sigma e^{\phi} p^{a} \epsilon_{a b c d} \bar{\theta}^{b} \bar{\partial} x^{c d},
$$

with a similar term linear in $e^{\bar{\phi}}$ and a higher order $e^{\phi} e^{\bar{\phi}}$ term. Let us analyze the structure of $S_{\phi}$. Just as in section 6 , we will work up to quartic order in fields (that is, in $p, \theta, e^{\phi}$, and their barred counterparts) and try to guess the general structure. Now, at least in the flat space case considered in section $4, p^{a}$ is one of the supercurrents and $\epsilon_{a b c d} \bar{\theta}^{b} \partial x^{c d}$ is the ghost-independent part of another supercurrent. Let us see if in the sigma model, 
the ghost coupling has a similar current-current form. In going to the sigma model, it is convenient to solve for $p^{a}$ by the equations of motion and write the above interaction as

$$
S_{\phi}=\frac{i}{8} \int d^{2} \sigma e^{\phi} \partial \bar{\theta}^{a} \epsilon_{a b c d} \bar{\theta}^{b} \partial x^{c d} .
$$

Here we have ignored the cubic corrections to $p^{a}=\partial \bar{\theta}^{a}$ because they would give sixth order terms in (8.2), which we are neglecting. Now, though this is not immediately apparent, (8.2) can actually be rewritten as

$$
S_{\phi}=-\frac{i}{2} \int d^{2} \sigma e^{\phi}\left(\partial \bar{\theta}^{a}-\frac{1}{4} \epsilon^{a b c d}\left(\bar{\theta}^{b} \partial x^{c d}-\partial \bar{\theta}^{b} x^{c d}\right)\right)\left(\overline{\partial \theta}^{a}-\frac{1}{4} \epsilon^{a e f g}\left(\bar{\theta}^{e} \bar{\partial} x^{f g}-\overline{\partial \theta}^{e} x^{f g}\right)\right) .
$$

We have added a variety of terms which vanish modulo fifth order expressions for various reasons. Some terms are explicitly fifth order in the fields. Also, the $e^{\phi} \partial \bar{\theta}^{a} \overline{\partial \theta}^{a}$ term is equivalent to a fifth order expression, after integrating by parts and using the fact that $\bar{\partial} e^{\phi}=0$ and that $\bar{\partial} \partial \theta^{a}=0$ modulo cubic terms. A term $e^{\phi} \partial \overline{\theta \partial}(\bar{\theta} x)$ is similarly fifth order after integration by parts.

Let us recall from equation (7.18) the form, up to quadratic order in fields, of the currents that generate the left supersymmetries of the sigma model:

$$
S_{L \alpha}^{a}=d \theta_{\alpha}^{a}-\frac{1}{4} \epsilon^{a b c d}\left(d \theta_{\alpha}^{b} x^{c d}-\theta_{\alpha}^{b} d x^{c d}\right)
$$

This has been written in a form that is manifestly invariant under the outer automorphism group $R=S U(2)$. For the time being, however, it will be more useful to relax this manifest invariance. We recall that $(\theta, \bar{\theta})$ are the upper and lower components of an $R$ doublet. We denote the upper and lower components of $S_{L}$ as $E, F$. We have up to second order

$$
\begin{aligned}
& E^{a}=d \theta^{a}-\frac{1}{4} \epsilon^{a b c d}\left(d \theta^{b} x^{c d}-\theta^{b} d x^{c d}\right) \\
& F^{a}=d \bar{\theta}^{a}-\frac{1}{4} \epsilon^{a b c d}\left(d \bar{\theta}^{b} x^{c d}-\bar{\theta}^{b} d x^{c d}\right) .
\end{aligned}
$$

Evidently then, we can write the coupling in (8.3) as

$$
S_{\phi}=-\frac{i}{2} \int d^{2} \sigma e^{\phi} F_{z}^{a} F_{z}^{a}=\frac{1}{2} \int d^{2} \sigma \epsilon^{i j} e^{\phi} F_{i}^{a} F_{j}^{a}=\frac{1}{2} \int e^{\phi} F^{a} \wedge F^{a} .
$$

We have introduced real coordinates by $z=\frac{1}{2}\left(\sigma^{1}+i \sigma^{2}\right)$, and used fermi statistics to express the coupling in terms of a wedge product of one-forms $F^{a}$. Where it will cause no confusion, we also write $E^{a}, F^{a}$ for the conserved charges obtained by integrating those 
currents; they are of course the upper and lower components of the supersymmetry doublet $S_{\alpha}^{a}$. We write $\eta_{+}^{a}, \eta_{-}^{a}$ for infinitesimal parameters generating left-moving supersymmetry. The left supersymmetry of the $S U^{\prime}(2 \mid 2)$ sigma model action

$$
S_{0}=\frac{1}{2} \int d^{2} \sigma\left\langle g^{-1} d g, g^{-1} d g\right\rangle
$$

studied in section 7 is thus

$$
\delta g=\left(\eta_{+}^{a} E_{a}+\eta_{-}^{a} F_{a}\right) g
$$

We will also denote as $K_{a b}$ the current generating the left action of the bosonic generators $K_{a b}$ of $S U^{\prime}(2 \mid 2)$ on $g$.

The ghost coupling in (8.3) is invariant under the right action of $S U^{\prime}(2 \mid 2)$ (the symmetry $g \rightarrow g B^{-1}$ ) since the left supercurrents that appear there are all right-invariant. It is also invariant under the left action of rotations (the bosonic symmetries in $S U^{\prime}(2 \mid 2)$ ), and under the left action of $F^{a}$. However, under the left action of $E^{a}, S_{\phi}$ is not invariant. Because of the commutation relation $\left\{E_{a}, F_{b}\right\}=\frac{1}{2} \epsilon_{a b c d} K^{c d}$, the variation of $S_{\phi}$ under a left supersymmetry is

$$
\delta S_{\phi}=\frac{1}{2} \int e^{\phi} \eta_{+}^{a} \epsilon_{a b c d} K^{c d} \wedge F^{b} .
$$

Now, recall from section 7 the Maurer-Cartan equation for the right-invariant oneforms on the $S U^{\prime}(2 \mid 2)$ manifold:

$$
d F_{a}=-\frac{1}{2} \epsilon_{a b c d} K^{b c} \wedge F^{d}
$$

and

$$
d E_{a}=-\frac{1}{2} \epsilon_{a b c d} K^{b c} \wedge E^{d}
$$

Using the formula for $d F_{a}$, we can write

$$
\delta S_{\phi}=-\int e^{\phi} \wedge \eta_{+a} d F^{a}
$$

The only hope of canceling this variation is to add ghost-dependent terms to the symmetry transformation (8.8). A clue comes from the flat case, where the supercharges have ghost dependent terms: $q_{a}^{-}=\oint p_{a}, q_{a}^{+}=\oint\left(e^{\phi} p_{a}-\frac{i}{2} \epsilon_{a b c d} \theta^{b} \partial x^{c d}\right)$. This suggests that in the flat case, to take the ghosts into account, the left supercurrents must be subjected to a $\phi$-dependent rotation which mixes $E$ and $F$. As will be shown below, the appropriate 
field-dependent rotation is $(E, F) \rightarrow\left(E+i e^{\phi} F, F\right)$. This is an $R$ transformation by the matrix

$$
W=\left(\begin{array}{cc}
1 & i e^{\phi} \\
0 & 1
\end{array}\right)
$$

This strongly suggests that we should modify the transformation law (8.8) under the left supersymmetries to be

$$
\delta g=\left(\eta_{+}^{a}\left(E_{a}+i e^{\phi} F_{a}\right)+\eta_{-}^{a} F_{a}\right) g .
$$

Now in general, under $\delta g=\epsilon g$, the sigma model action $S=\int d^{2} \sigma \frac{1}{2}\left\langle g^{-1} \partial_{i} g, g^{-1} \partial^{i} g\right\rangle$ changes by $\delta S=\int d^{2} \sigma\left\langle\partial_{i} \epsilon, \partial^{i} g g^{-1}\right\rangle$. For the transformation in (8.14), the variation is

$$
\delta S=i \int d^{2} \sigma \partial_{i} e^{\phi} \eta_{+}^{a} F_{i}^{a} .
$$

Because $e^{\phi}$ is holomorphic, we have $\left(\partial_{i}+i \epsilon_{i j} \partial_{j}\right) e^{\phi}=0$. Using this fact and integrating by parts, we get

$$
\delta S=\int d^{2} \sigma e^{\phi} \eta_{+}^{a} \epsilon^{i j} \partial_{i} F_{j}^{a} .
$$

Comparing this with (8.12), we see that to this order, the total action $S+S_{\phi}$ is invariant under the modified spacetime supersymmetry transformation (8.14).

\subsection{Left And Right}

One strange thing about this result is that we have modified the left action of $S U^{\prime}(2 \mid 2)$ on itself, but not the right action. However, by a change of variables we could reverse this result, modifying the right action but not the left action. Indeed, suppose that we make the $R$-transformation that is inverse to $W$ :

$$
W^{-1}=\left(\begin{array}{cc}
1 & -i e^{\phi} \\
0 & 1
\end{array}\right)
$$

This transforms the modified supersymmetries of (8.14) into the standard ones - showing in particular that the modified supersymmetries do possess the standard commutation relations. However, since there is only one $R$ symmetry group that acts on all degrees of freedom including the left and right supersymmetries, this transformation will turn the right action of supersymmetry into

$$
\delta g=g\left(\eta_{+}^{a}\left(E^{a}-i e^{\phi} F^{a}\right)+\eta_{-}^{a} F^{a}\right) .
$$


Thus, we can put the left action or the right action of $S U^{\prime}(2 \mid 2)$ in the standard form, but not both.

The left currents in $S_{\phi}$ are dictated by the exotic action of the left supersymmetries in (8.18). The similarity transformation that "straightens out" the left supersymmetries and makes the right supersymmetries exotic will therefore replace the left currents in $S_{\phi}$ by right currents.

This situation is quite unfamiliar. The model possesses $G \times G$ symmetry. One can pick variables to make the action of either copy of $G$ standard. But one cannot simultaneously put the action of both copies of $G$ in a standard form.

We will show explicitly how to change variables so that the ghost couplings are written in terms of right currents rather than left currents. Under the $R$-transformation of (8.17), the sigma model action of (8.7) transforms to

$$
\delta S=i \int d^{2} \sigma\left(\partial_{i} e^{\phi}\right) r_{i}^{22}
$$

where $r_{i}^{\alpha \beta}$ is the conserved current associated to the $R$-transformation. Instead of computing $r_{i}^{\alpha \beta}$ directly using the Noether method, we shall compute it indirectly from its commutation relations with the $S U^{\prime}(2 \mid 2)$ charges. If $\oint r^{\alpha \beta}$ is the $R$-charge, then the only non-zero commutation relations are with the left and right susy charges which gives

$$
\begin{aligned}
& {\left[\oint r^{\alpha \beta}, \oint S_{L a}^{\gamma}\right]=\frac{1}{2}\left(\epsilon^{\beta \gamma} \oint S_{L a}^{\alpha}+\epsilon^{\alpha \gamma} \oint S_{L a}^{\beta}\right),} \\
& {\left[\oint r^{\alpha \beta}, \oint S_{R a}^{\gamma}\right]=\frac{1}{2}\left(\epsilon^{\beta \gamma} \oint S_{R a}^{\alpha}+\epsilon^{\alpha \gamma} \oint S_{R a}^{\beta}\right) .}
\end{aligned}
$$

Using (7.25) and (7.26), one sees that $\oint r^{\alpha \beta}$ has the same commutation relations as $-\frac{1}{2} \oint\left(\lambda_{L}^{\alpha \beta}+\lambda_{R}^{\alpha \beta}\right)$. Similarly, $d r^{\alpha \beta}$ has the same commutation relations as $-\frac{1}{2}\left(\omega_{L}^{\alpha \beta}+\omega_{R}^{\alpha \beta}\right)$. Since any two-form which commutes with all the $S U^{\prime}(2 \mid 2) \times S U^{\prime}(2 \mid 2)$ transformations must vanish, $d r^{\alpha \beta}+\frac{1}{2}\left(\omega_{L}^{\alpha \beta}+\omega_{R}^{\alpha \beta}\right)$ must vanish, i.e.

$$
d r^{\alpha \beta}=-\frac{1}{2}\left(\omega_{L}^{\alpha \beta}+\omega_{R}^{\alpha \beta}\right) .
$$

Since $\bar{\partial} e^{\phi}=0,(8.19)$ is equal to

$$
\begin{gathered}
\delta S=\int d^{2} \sigma e^{\phi} \epsilon^{i j} \partial_{i} r_{j}^{22}=\int e^{\phi} \wedge d r_{j}^{22} \\
=-\frac{1}{2} \int e^{\phi}\left(F_{L}^{a} \wedge F_{L}^{a}+F_{R}^{a} \wedge F_{R}^{a}\right) .
\end{gathered}
$$

So the $R$-transformation of (8.7) changes the ghost-coupling from $S_{\phi}=\frac{1}{2} \int e^{\phi} F_{L}^{a} \wedge F_{L}^{a}$ to $S_{\phi}=-\frac{1}{2} \int e^{\phi} F_{R}^{a} \wedge F_{R}^{a}$, which now depends only on left-invariant currents as expected. 


\subsection{Incorporation Of $e^{\bar{\phi}}$}

Having understood the basic structure, it is now not so difficult to give a full description of the ghost couplings.

First, consider the term linear in $e^{\bar{\phi}}$ (with no copies of $e^{\phi}$ ). By manipulations quite similar to what we have seen, this coupling can be put in the form

$$
S_{\bar{\phi}}=-\frac{1}{2} \int d^{2} \sigma e^{\bar{\phi}} E^{a} \wedge E^{a} .
$$

Its variation, furthermore, can be canceled by modifying (8.14) so that the left supersymmetry transformations are

$$
\delta g=\left(\eta_{+}^{a}\left(E_{a}+i e^{\phi} F_{a}\right)+\eta_{-}^{a}\left(F_{a}-i e^{\bar{\phi}} E_{a}\right)\right) g
$$

Of course, just as in the discussion of $S_{\phi}$, we could make an $R$-symmetry transformation to remove this correction to the left supersymmetry transformations, at the cost of adding a similar correction to the right supersymmetry transformations. $S_{\bar{\phi}}$ would then be expressed in terms of right currents.

The action $S+S_{\phi}+S_{\bar{\phi}}$ is invariant under the transformation (8.24), up to terms of order $e^{\phi} e^{\bar{\phi}}$. To cancel those terms, it is necessary to add to the action further terms proportional to $e^{\phi} e^{\bar{\phi}}$. This should come as no surprise, because as we saw in section 6 , the vertex operator for the leading order deformation from flat space to $\mathrm{AdS}_{3} \times \mathbf{S}^{3}$ contains a term proportional to $e^{\phi} e^{\bar{\phi}}$. As we will see presently, there actually are further terms $e^{n \phi} e^{m \bar{\phi}}$ with all positive integers $n, m$ satisfying $|n-m| \leq 1$. Luckily, with the experience we have gained so far, it is possible to guess the general structure.

\subsection{Exact Non-Linear Ghost Couplings}

Up to this order in $e^{\phi}$ and $e^{\bar{\phi}}$, the action

$$
S=S_{0}+\frac{1}{2} \int e^{\phi} F^{a} \wedge F^{a}-\frac{1}{2} \int e^{\bar{\phi}} E^{a} \wedge E^{a}
$$

is invariant under the ghost-modified left supersymmetries of (8.24), where $S_{0}$ is the $S U^{\prime}(2 \mid 2)$ sigma model action of (8.7). We will now generalize (8.25) and (8.24) in a manner that leaves the action invariant to all orders in $e^{\phi}$ and $e^{\bar{\phi}}$.

The generalization of the ghost-modified left supersymmetries will be defined by

$$
\delta g=\left(\eta^{a}{ }_{I} v_{\alpha}^{I} S^{\alpha a}\right) g
$$


where $\eta_{I}^{a}$ are constant anticommuting parameters, $v_{\alpha}^{I}$ is a rotation matrix that depends on $e^{\phi}$ and $e^{\bar{\phi}}$ in a fashion which will be determined, and we recall that $S^{\alpha a}=\left(E^{a}, F^{a}\right)$ is the doublet of supersymmetry generators. To lowest order in $e^{\phi}$ and $e^{\bar{\phi}}$, the matrix elements of $v$ are given by

$$
v_{1}^{+}=1, \quad v_{2}^{+}=i e^{\phi}, \quad v_{1}^{-}=-i e^{\bar{\phi}}, \quad v_{2}^{-}=1,
$$

where to avoid confusing the different types of index we let $\alpha$ take the values 1,2 and $I$ takes the values,+- . Under the transformation of (8.26), the sigma model action $S_{0}$ transforms as

$$
\delta S_{0}=\int d^{2} \sigma \eta_{I}^{a}\left(S^{\alpha a} \partial_{i} v_{\alpha}^{I} \cdot\right)
$$

So one would like to construct a generalization of (8.25) such that the transformation of the $\phi$-dependent terms in the action cancels (8.28). This generalization can be found by considering the action $S=S_{0}+S_{1}$ where $S_{0}$ is the sigma model action of (8.7) and

$$
S_{1}=\int \sum_{\alpha, \beta=1,2} c_{\alpha \beta} S^{a \alpha} \wedge S^{a \beta}
$$

Here $c_{\alpha \beta}=c_{\beta \alpha}$; the $c^{\prime}$ s are functions of $e^{\phi}$ and $e^{\bar{\phi}}$ that will be determined. We recall also that $S^{a \alpha}$ denotes either the supersymmetry generators or the corresponding left currents, which are defined as

$$
\left\langle S^{a \alpha}, d g g^{-1}\right\rangle
$$

Note that the constant $\phi$-independent parts of $c^{A B}$ can be chosen arbitrarily since, as shown in section 7 ,

$$
\int S^{a \alpha} \wedge S^{a \beta}=0
$$

Ignoring this constant part, the lowest order $e^{\phi}$ contributions were computed earlier to be $c_{11}=e^{\bar{\phi}}, c_{22}=-e^{\phi}$ and $c_{12}=0$.

Under the modified left susy transformation of (8.26), the currents transform as

$$
\delta S^{a \alpha}=-\eta_{I}^{a} d v^{I \alpha}-\frac{1}{2} \epsilon^{a b c d} \eta_{I b} K_{c d} v^{I \alpha}
$$

This formula is obtained directly from the definition (8.30) of the supercurrents. Under $\delta g=\epsilon g$, the variation of the supercurrent has two terms, one proportional to a derivative of $\epsilon$ and one not; these give the two terms in (8.31). 
So under (8.26), $S_{1}$ transforms as

$$
\begin{aligned}
\delta S_{1} & =2 \int c_{\alpha \beta}\left(-\eta_{I}^{a} d v^{I \alpha} S^{a \beta}-\frac{1}{2} \epsilon_{a b c d} \eta_{I}^{b} v^{I \alpha} K^{c d} S^{a \beta}\right) \\
& =-2 \int c_{\alpha \beta} \eta_{I}^{a}\left(d v^{I \alpha} S^{\beta}+v^{I \alpha} d S^{a \beta}\right)=2 \int d c_{\alpha \beta} \eta_{I}^{a} v^{I \alpha} S^{a \beta}
\end{aligned}
$$

where $v^{I \alpha}=\epsilon^{\alpha \gamma} v_{\gamma}^{I}$. Here we have used the Maurer-Cartan equations and integrated by parts.

So to cancel the transformation of 8.28 for all $\eta_{I}^{a}$, we require the relation

$$
\partial_{i} v_{\alpha}^{I}=-2 \epsilon_{i j} v_{\beta}^{I} \epsilon^{\beta \gamma} \partial_{j} c_{\gamma \alpha}
$$

We define a $2 \times 2$ matrix

$$
C_{\beta}^{\alpha}=\epsilon^{\alpha \gamma} c_{\gamma \beta}
$$

Likewise, we regard $v_{\alpha}^{I}$ as the matrix elements of a $2 \times 2$ matrix $V$. The equation then reads

$$
V^{-1} \partial_{i} V=-2 \epsilon_{i j} \partial_{j} C
$$

As $C$ is traceless, the determinant of $V$ is constant. In terms of a complex coordinate $z=\frac{1}{2}\left(\sigma^{1}+i \sigma^{2}\right)$, the equation for $V$ and $C$ becomes

$$
\begin{aligned}
& V^{-1} \partial V=-2 i \partial C \\
& V^{-1} \bar{\partial} V=2 i \bar{\partial} C
\end{aligned}
$$

with $\partial=\partial_{z}, \bar{\partial}=\partial_{\bar{z}}$. This implies that

$$
\partial \bar{\partial} C=\frac{1}{2}(\bar{\partial} \partial C+\partial \bar{\partial} C)=-\frac{i}{4}\left[V^{-1} \bar{\partial} V, V^{-1} \partial V\right]=-i[\bar{\partial} C, \partial C] .
$$

So we are looking for solutions to $\partial \bar{\partial} C=-i[\bar{\partial} C, \partial C]$ such that, ignoring contant $\phi$ independent terms, $c_{11}=-\frac{1}{2} e^{\bar{\phi}}, c_{22}=\frac{1}{2} e^{\phi}$, and $c_{12}=0$ when $e^{\phi}$ is small. One can solve these conditions explicitly to find that

$$
C=\frac{1}{1-\frac{1}{4} e^{\phi+\bar{\phi}}}\left(\begin{array}{cc}
i & -\frac{1}{2} e^{\phi} \\
-\frac{1}{2} e^{\bar{\phi}} & -i
\end{array}\right) .
$$

So the exact expression for the action is $S=S_{0}+S_{1}$ where

$$
S_{1}=\int\left(1-\frac{1}{4} e^{\phi+\bar{\phi}}\right)^{-1}\left(\frac{1}{2} e^{\phi} F^{a} \wedge F^{a}-\frac{1}{2} e^{\bar{\phi}} E^{a} \wedge E^{a}-2 i E^{a} \wedge F^{a}\right) .
$$


One can now look for a matrix $V$ satisfying (8.36) (where $C$ is given by (8.38)) such that (8.27) is satisfied when $e^{\phi}$ is small. Once again, one can explicitly solve these conditions to find that

$$
V=\frac{1}{1-\frac{1}{4} e^{\phi+\bar{\phi}}}\left(\begin{array}{cc}
1+\frac{1}{4} e^{\phi+\bar{\phi}} & i e^{\phi} \\
-i e^{\bar{\phi}} & 1+\frac{1}{4} e^{\phi+\bar{\phi}}
\end{array}\right)
$$

\subsection{Exact Ghost Couplings in Presence of Wess-Zumino Term}

One can also ask what happens to the ghost couplings in $S_{1}$ after adding a WessZumino term to $S_{0}$ with coupling parameter $N$. For this, we restore the coupling parameter $f$ (which we have suppressed so far in this section) and introduce also the Wess-Zumino coupling $k$. So the action is

$$
S=\frac{1}{f^{2}}\left(S_{0}+S_{1}\right)+k S_{W Z}=\frac{1}{f^{2}}\left(S_{0}+S_{1}+N S_{W Z}\right)
$$

where $N=k f^{2}$ and $S_{W Z}=\frac{i}{2} \int d^{3} \sigma\left\langle d g g^{-1},\left[d g g^{-1}, d g g^{-1}\right]\right\rangle$.17 In this case, the transformation of $S_{0}+N S_{W Z}$ under the left supersymmetry transformation of (8.26) is given by

$$
\delta S_{0}+N \delta S_{W Z}=\int d^{2} \sigma \eta_{I}^{a}\left(\partial_{i} v_{\alpha}^{I} S_{i}^{a \alpha}+i N \epsilon^{i j} \partial_{i} v_{\alpha}^{I} S_{j}^{a \alpha}\right)
$$

Here we have kept the definition $S^{a \alpha}=\left\langle S^{a \alpha}, d g g^{-1}\right\rangle$, but with $N \neq 0$, the left supersymmetry currents are actually the combinations in (8.42): $\widetilde{S}_{i}^{a \alpha}=S_{i}^{a \alpha}+i N \epsilon_{i j} S_{j}^{a \alpha}$. Defining $c_{A B}, V$ and $C$ as above, and requiring that $\delta S_{1}$ cancels the transformation of (8.42), one finds that the equation satisfied by $C$ is modified to

$$
\partial C=\frac{i}{2}(1+N) V^{-1} \partial V, \quad \bar{\partial} C=-\frac{i}{2}(1-N) V^{-1} \bar{\partial} V .
$$

This implies that

$$
\begin{gathered}
\partial \bar{\partial} C=\frac{1}{2}[(1-N) \bar{\partial} \partial C+(1+N) \partial \bar{\partial} C]=\frac{i}{4}(N-1)(N+1)\left[V^{-1} \bar{\partial} V, V^{-1} \partial V\right] \\
=-i[\bar{\partial} C, \partial C] .
\end{gathered}
$$

So the equation for $C$ is independent of $N$.

The initial conditions for this equation, however, depend on $N$. To determine them, we should be a bit more precise about how the derivation in section 6 generalizes in the

17 We take Euclidean signature for the worldsheet and hence include a factor of $i$ in $S_{W Z}$. 
presence of both RR and NS flux. We start with the flat model as presented in section 4 , and then perturb the Lagrangian by RR and NS vertex operators with coefficients $f_{R R}$ and $f_{N S}$ :

$$
L \rightarrow L+f_{R R} \int V_{R R}+f_{N S} \int V_{N S}
$$

In second order, both the RR and NS fluxes give back reaction on the metric, and the Lagrangian will require a further correction

$$
\left(f_{R R}^{2}+f_{N S}^{2}\right) \int d^{2} \sigma \frac{1}{24} \epsilon_{a b c d} x^{a e} x^{b f} \partial x^{c e} \bar{\partial} x^{d f}+\ldots
$$

The coefficient of this term determines, after some rescalings of fields, the sigma model radius $1 / f$. In other words, if we let $f=\sqrt{f_{R R}^{2}+f_{N S}^{2}}$, and rescale $x$ and the $\theta$ 's by a factor of $f^{-1}$, then the ghost-free part of the action gets an overall scale $1 / f^{2}$, as in section 6. Since the $e^{\phi}$ and $e^{\bar{\phi}}$ couplings arise in linear order only from $f_{R R}$, they are proportional after the rescaling to $f_{R R} / f$ times the expression at $f_{N S}=0$. Thus, the term in $S_{1}$ linear in $e^{\phi}$ and $e^{\bar{\phi}}$ is multiplied by $f_{R R} / f$ from what it is in the pure RR case. Also, note that a Wess-Zumino coupling comes only from $V_{N S}$, and hence after the rescaling, $N=f_{N S} / f$.

The net effect is that turning on NS flux multiplies the linear contribution to $C$ by a factor $f_{R R} / f=\sqrt{1-N^{2}}$. Since this factor appears with $e^{\phi}, e^{\bar{\phi}}$ only in the combinations $\sqrt{1-N^{2}} e^{\phi}$ and $\sqrt{1-N^{2}} e^{\bar{\phi}}$, it could be removed by adding a constant to $\phi$ and $\bar{\phi}$, an operation under which the equation for $C$ is invariant. Up to such a shift, the result for $C$ is independent of $N$. If we prefer not to shift $\phi$ and $\bar{\phi}$, then the general solution for $C$ is

$$
C\left(e^{\phi}, e^{\bar{\phi}}, N\right)=C_{0}\left(\sqrt{1-N^{2}} e^{\phi}, \sqrt{1-N^{2}} e^{\bar{\phi}}\right)
$$

where $C_{0}$ is the solution found above at $N=0$.

There is an important benefit of not shifting $\phi$ and $\bar{\phi}$ to eliminate the $N$ dependence. Because of the background charge in the worldsheet Lagrangian, such a shift would change the string coupling constant by an amount that would diverge if we go to the points $N= \pm 1$. If we want to vary $N$ keeping the string coupling constant fixed, we should use the form of the $C$ matrix in (8.46).

Equation (8.46) shows that going beyond the WZW point, that is to $|N|>1$, would change the reality properties of the solution. (For $|N|<1, C$ is hermitian if we consider $\bar{\phi}$ the complex conjugate of $\phi$, but that fails for $|N|>1$.) In terms of our discussion in section 7 , this is related to the fact that the fluxes are no longer real if $|k|>1 / f^{2}$. 
To study the limit as $N \rightarrow 1$, a subtle choice of shifting $\phi$ and $\bar{\phi}$ is useful. We counter-rotate $\phi$ and $\bar{\phi}$ :

$$
\begin{aligned}
e^{\phi} & \rightarrow-\frac{2}{\sqrt{1-N^{2}}} e^{\phi} \\
e^{\bar{\phi}} & \rightarrow-\frac{\sqrt{1-N^{2}}}{2} e^{\bar{\phi}} .
\end{aligned}
$$

Factors of -2 have been included to put the resulting formulas in the nicest form. This rescaling has no effect on the string coupling constant, since the background charge effects cancel between $\phi$ and $\bar{\phi}$. For $N$ near 1, we now get

$$
C=\left(1+\frac{1-N^{2}}{4} e^{\phi+\bar{\phi}}\right)\left(\begin{array}{cc}
i & e^{\phi} \\
\frac{1-N^{2}}{4} e^{\bar{\phi}} & -i
\end{array}\right)+O\left((1-N)^{2}\right) .
$$

We have included terms of order $(1-N)$ since these are needed to determine the solution for $V$. We now compute that to leading order near $N=1$,

$$
\begin{aligned}
\partial C & =\partial \phi\left(\begin{array}{cc}
0 & e^{\phi} \\
0 & 0
\end{array}\right) \\
\bar{\partial} C & =\frac{1}{2}(1-N) \bar{\partial} \bar{\phi}\left(\begin{array}{cc}
i e^{\phi+\bar{\phi}} & e^{2 \phi+\bar{\phi}} \\
e^{\bar{\phi}} & -i e^{\phi+\bar{\phi}}
\end{array}\right) .
\end{aligned}
$$

Inserting these formulas in (8.43), we find that the limit of $V$ for $N \rightarrow 1$ is

$$
V=\left(\begin{array}{cc}
1 & 0 \\
i e^{\bar{\phi}} & 1
\end{array}\right)\left(\begin{array}{cc}
1 & -i e^{\phi} \\
0 & 1
\end{array}\right) .
$$

In the formalism that we have used up to this point, the right supercurrents are the standard ones, and the left ones are rotated by $V . N=1$ is in fact the WZW point, at which the left currents are anti-holomorphic and the right currents are holomorphic. A more natural description at the WZW point is one in which the left currents are rotated by an anti-holomorphic factor, and the right currents by a holomorphic factor. To get to such a description, we make an $R$ transformation by the inverse of the second factor in $V$, to go to a description in which the anti-holomorphic currents are rotated from the standard ones by a factor

$$
\left(\begin{array}{cc}
1 & 0 \\
i e^{\bar{\phi}} & 1
\end{array}\right)
$$

and the holomorphic currents are instead rotated by

$$
\left(\begin{array}{cc}
1 & i e^{\phi} \\
0 & 1
\end{array}\right)
$$


It will now be shown that this $R$-transformation removes the ghost couplings derived from (8.48), so we get a pure WZW model of $S U^{\prime}(2 \mid 2)$, with the currents rotated as just indicated. This description, which matches nicely with the treatment of the flat case in section 4, will be our starting point in section 10, where we examine $S U^{\prime}(2 \mid 2)$ current algebra.

So we need to show that

$$
\delta\left(S_{0}+N S_{W Z}\right)+\delta\left(S_{\phi}\right)=-S_{\phi}
$$

under the $R$-transformation of (8.52) where $S_{\phi}=-\int e^{\phi} F^{a} \wedge F^{a}$. We will organize the proof as follows. We will show that the first order variation of $S_{0}+N S_{W Z}-$ that is, the term of order $e^{\phi}-$ is

$$
\delta_{1}\left(S_{0}+N S_{W Z}\right)=-S_{\phi}
$$

This is the result we want to first order in $e^{\phi}$. Moreover, we will prove that there are no higher order terms (proportional to $e^{n \phi}$ for $\left.n>1\right)$ in $\delta\left(S_{0}+N S_{W Z}\right)$. Combining this last fact with (8.54), which identifies $S_{\phi}$ as the first order variation of $S_{0}+N S_{W Z}$, it follows that $\delta S_{\phi}=0$. Hence the left hand side of (8.53) is linear in $e^{\phi}$, and (8.54) is equivalent to (8.53).

To show (8.54), note first that

$$
\delta_{1}\left(S_{0}+N S_{W Z}\right)=-i \int d^{2} \sigma\left(\partial_{i} e^{\phi}\right) \widetilde{r}_{i}^{22}=i \int d^{2} \sigma e^{\phi} \partial_{z} \widetilde{r}_{\bar{z}}^{22}
$$

where $\widetilde{r}_{i}^{\alpha \beta}$ is the conserved current of the WZW model associated to the $R$-transformation. At this stage, we can prove that there can be no higher order variation of $S_{0}+N S_{W Z}$. Indeed, since two strictly upper triangular $2 \times 2$ matrices commute, the $R$-transformation we are making, if the coefficient $e^{\phi}$ is constant, would commute with the current $\widetilde{r}_{\bar{z}}^{22}$ that generates an upper triangular $R$ transformation. A variation of $\widetilde{r}_{\bar{z}}^{22}$ proportional to derivatives of $e^{\phi}$ would, on symmetry and dimensional grounds, be proportional to $\bar{\partial} e^{\phi}=0$. So $\delta\left(S_{0}+N S_{W Z}\right)=\delta_{1}\left(S_{0}+N S_{W Z}\right)$. As we discussed in the last paragraph, it follows that (8.54) and (8.53) are equivalent.

As in subsection (8.2), we shall compute $\widetilde{r}_{i}^{\alpha \beta}$ indirectly from its commutation relations with the $S U^{\prime}(2 \mid 2)$ charges

$$
\left[\oint \widetilde{r}^{\alpha \beta}, \oint \widetilde{S}_{L a}^{\gamma}\right]=\frac{1}{2}\left(\epsilon^{\beta \gamma} \oint \widetilde{S}_{L a}^{\alpha}+\epsilon^{\alpha \gamma} \oint \widetilde{S}_{L a}^{\beta}\right)
$$




$$
\left[\oint \widetilde{r}^{\alpha \beta}, \oint \widetilde{S}_{R a}^{\gamma}\right]=\frac{1}{2}\left(\epsilon^{\beta \gamma} \oint \widetilde{S}_{R a}^{\alpha}+\epsilon^{\alpha \gamma} \oint \widetilde{S}_{R a}^{\beta}\right)
$$

which implies that

$$
\begin{aligned}
& {\left[\widetilde{r}^{\alpha \beta}, \oint \widetilde{S}_{L a}^{\gamma}\right]=\frac{1}{2}\left(\epsilon^{\beta \gamma} \widetilde{S}_{L a}^{\alpha}+\epsilon^{\alpha \gamma} \widetilde{S}_{L a}^{\beta}\right)+d M_{a}^{\alpha \beta \gamma},} \\
& {\left[\widetilde{r}^{\alpha \beta}, \oint \widetilde{S}_{R a}^{\gamma}\right]=\frac{1}{2}\left(\epsilon^{\beta \gamma} \widetilde{S}_{R a}^{\alpha}+\epsilon^{\alpha \gamma} \widetilde{S}_{R a}^{\beta}\right)+d N_{a}^{\alpha \beta \gamma},}
\end{aligned}
$$

for some $M_{a}^{\alpha \beta \gamma}$ and $N_{\alpha \beta \gamma}$. Note that the susy charges and currents contain tilde's since they include the contribution from the Wess-Zumino term.

Since $\widetilde{S}_{L a}^{\alpha}$ has only a $\bar{z}$ component and $\widetilde{S}_{R a}^{\alpha}$ has only a $z$ component, (8.57) implies that

$$
\begin{gathered}
{\left[\widetilde{r}_{\bar{z}}^{\alpha \beta}, \oint \widetilde{S}_{L a}^{\gamma}\right]=\frac{1}{2}\left(\epsilon^{\beta \gamma} \widetilde{S}_{L a \bar{z}}^{\alpha}+\epsilon^{\alpha \gamma} \widetilde{S}_{L a \bar{z}}^{\beta}\right)+\bar{\partial} M_{a}^{\alpha \beta \gamma}} \\
{\left[\widetilde{r}_{\bar{z}}^{\alpha \beta}, \oint \widetilde{S}_{R a}^{\gamma}\right]=\bar{\partial} N_{a}^{\alpha \beta \gamma} .}
\end{gathered}
$$

So, ignoring the $\bar{\partial}$ terms, $\widetilde{r}_{\bar{z}}^{\alpha \beta}$ has the same commutation relation as $-\lambda_{L \bar{z}}^{\alpha \beta}$ of (7.25) since $\widetilde{S}_{L a \bar{z}}^{\alpha}=2 S_{L a \bar{z}}^{\alpha}$ when $N=1$. Therefore, $\widetilde{r}_{\bar{z}}^{\alpha \beta}+\lambda_{L \bar{z}}^{\alpha \beta}$ is $S U^{\prime}(2 \mid 2) \times S U^{\prime}(2 \mid 2)$-invariant up to $\bar{\partial}$ terms, which implies that

$$
\widetilde{r}_{\bar{z}}^{\alpha \beta}=-\lambda_{L \bar{z}}^{\alpha \beta}+\bar{\partial} f^{\alpha \beta}
$$

for some $f^{\alpha \beta}$. Using (8.59) and $\bar{\partial} e^{\phi}=0$, (8.55) implies that

$$
\delta\left(S_{0}+N S_{W Z}\right)=-i \int d^{2} \sigma e^{\phi} \partial_{z} \lambda_{L \bar{z}}^{22}=\int e^{\phi} \wedge \omega_{L}^{22}=\int e^{\phi} F_{L}^{a} \wedge F_{L}^{a}=-S_{\phi} .
$$

This completes the proof.

\subsection{Quantum Treatment And Conformal Invariance}

The above analysis of the supersymmetry of the ghost couplings was really based on a classical manipulation. One may question whether there are quantum anomalies that spoil supersymmetry.

First of all, there is no problem in maintaining invariance under the right action of $S U^{\prime}(2 \mid 2)$, since this is preserved, for example, by Pauli-Villars regularization. Likewise, Pauli-Villars regularization preserves the symmetry under the bosonic part of $S U^{\prime}(2 \mid 2)$, acting on the left. The question is to preserve the left supersymmetries.

Let us suppose that, in some regularization, $c$ and $v$ have been chosen so that the supersymmetric variation of the action vanishes up to $k^{\text {th }}$ order in $e^{\phi}$ and $e^{\bar{\phi}}$, that is 
including all terms $e^{a \phi+b \bar{\phi}}$ with $a+b \leq k$. We also assume that $c$ and $v$ to this order are polynomials of order $k$ in $e^{\phi}$ and $e^{\bar{\phi}}$. We will show that $c$ and $v$ can be corrected by $k+1^{\text {th }}$ order polynomials to cancel the variation in that order. The analysis could be taken as a substitute for the explicit solution of the classical equations that we have given above.

An important part of the structure is that since $\phi$ and $\bar{\phi}$ enter only in the combinations $e^{\phi}$ and $e^{\bar{\phi}}$, which have no short distance singularities, $\phi$ and $\bar{\phi}$ can be treated in making the analysis as $c$-number functions that obey $\bar{\partial} \phi=\partial \bar{\phi}=0$. Also, if $\phi$ and $\bar{\phi}$ are constant, the whole deformation that we have constructed collapses. That is because, as explained in section 7, the two forms $S^{a \alpha} \wedge S^{a \beta}$ can be written

$$
S^{a \alpha} \wedge S^{a \beta}=d \lambda^{\alpha \beta}
$$

for some $\lambda$. Hence the interaction $S_{1}$ is

$$
S_{1}=-\int d c_{\alpha \beta} \wedge \lambda^{\alpha \beta}
$$

where we can think of $\lambda$ as a quantum field operator of dimension one. This shows that the corrections to the sigma model action $S_{0}$ vanish if $c$ is constant and in general are "soft" or superrenormalizable interactions. We will not actually use this way of writing $S_{1}$ explicitly, because there is no nice choice of $\lambda$ (for instance, one cannot pick $\lambda$ to be right-invariant), but nevertheless this fact underlies the arguments that follow. Because $S_{1}=0$ if $\phi$ and $\bar{\phi}$ are constant, it follows that any anomaly is proportional to $\partial_{z} \phi$ or $\partial_{\bar{z}} \bar{\phi}$. Since $\phi$ and $\bar{\phi}$ appear only via powers of $e^{\phi}$ or $e^{\bar{\phi}}$, any $\partial_{z} \phi$ term multiplies $e^{a \phi}$ for some $a>0$, and any $\partial_{\bar{z}} \bar{\phi}$ term multiplies $e^{b \bar{\phi}}$ for some $b>0$.

Let $\widetilde{S}^{a I}=v_{\alpha}^{I} S^{a \alpha}$ be the rotated supercurrents that generate the left supersymmetry to order $k$. Thus, the divergence of $\widetilde{S}^{a I}$ is of order $k+1$ in $e^{\phi}, e^{\bar{\phi}}$. This divergence is of the form

$$
\partial_{i} \widetilde{S}_{i}^{a I}=\mathcal{O}^{a I}
$$

where $\mathcal{O}^{a I}$ has the following properties. It is right-invariant and of dimension two since $\widetilde{S}$ is right-invariant and of dimension one; it is proportional to $\partial \phi$ or $\bar{\partial} \phi$ since there is no anomaly if these vanish; and because of the $a$ index of the current, it transforms as a vector under the "rotation" subgroup of $S U^{\prime}(2 \mid 2)$. Any operator with these properties is a linear 
combination of the left supersymmetry currents times $\partial \phi$ or $\bar{\partial} \phi .18$ The divergence of the currents is therefore of this form, and hence so is the variation of the action $S_{0}+S_{1}$ under a supersymmetry generated by $\widetilde{S}^{a I}$ :

$$
\delta_{a I}\left(S_{0}+S_{1}\right)=\int d^{2} \sigma\left(\partial_{z} \phi f_{I J} \widetilde{S}_{\bar{z} a}^{J}+\partial_{\bar{z}} \bar{\phi} g_{I J} \widetilde{S}_{z a}^{J}\right)
$$

Here $f_{I J}$ and $g_{I J}$ are unknown functions of $e^{\phi}$ and $e^{\bar{\phi}}$. A "Wess-Zumino consistency condition" 19 shows that

$$
f_{I J}=f_{J I}, \quad g_{I J}=g_{J I} .
$$

Furthermore, $f_{I J}$ is divisible by $e^{\phi}$, since, as noted at the end of the last paragraph $\phi$ only appears in this form, so the $\partial \phi$ term in (8.63) is really a sum of terms $\partial \phi e^{a \phi}$ with $a>0$; similarly, $g_{I J}$ is divisible by $e^{\bar{\phi}}$. Since $\partial \phi e^{a \phi}=\partial\left(e^{a \phi} / a\right)$ and similarly for $\bar{\phi}$, we can write $f_{I J}=\partial F_{I J}, g_{I J}=\bar{\partial} G_{I J}$, where $F$ and $G$ like $f$ and $g$ are polynomials in $e^{\phi}, e^{\bar{\phi}}$. The anomaly is therefore

$$
\delta_{a I}\left(S_{0}+S_{1}\right)=\int d^{2} \sigma\left(\partial_{z} F_{I J} \widetilde{S}_{\bar{z} a}^{J}+\partial_{\bar{z}} G_{I J} \widetilde{S}_{z a}^{J}\right)
$$

Now we write $F=H+K, G=H-K$, and of course we are interested in canceling the term of order $k+1$ (in $e^{\phi}$ and $e^{\bar{\phi}}$ ) in $H$ and $K$. The contribution proportional to $K$ can be canceled via a $k+1^{t h}$ order correction to $c$, and the contribution proportional to $H$ can be canceled via a $k+1^{\text {th }}$ order contribution to $v$.

This shows that spacetime supersymmetry is not spoiled by any quantum anomalies; at most such anomalies modify the classical expressions for $c$ and $v$.

18 Any dimension one classical operator is $\lambda_{A} \partial_{i} \Phi^{A}$, where $\Phi^{A}$ are the coordinates on the group manifold and $\lambda=\lambda_{A} d \Phi^{A}$ is a one-form on the group manifold. The operator in question is right-invariant if and only if $\lambda$ is right-invariant; but the right-invariant one-forms are associated precisely with the left currents.

19 One applies $\delta_{b J}$ to 8.63 and uses the fact that $\left\{\delta_{b J}, \delta_{a I}\right\}\left(S_{0}+S_{1}\right)=0$ since $\left\{\delta_{b J}, \delta_{a I}\right\}$ is a generator of rotations and the action is manifestly rotation-invariant. But in evaluating $\delta_{b J}\left(S_{0}+S_{1}\right)$ from the formula $(8.63)$, the action of $\delta_{b J}$ on the right and side is only via the action of supersymmetry on the supercurrents $\widetilde{S}, \delta_{b J} \widetilde{S}_{a I} \sim \epsilon_{I J} \epsilon_{a b c d} K^{c d}$. Because of the $\epsilon_{I J}$, $\left\{\delta_{b J}, \delta_{a I}\right\}\left(S_{0}+S_{1}\right)=0$ holds if and only if $f$ and $g$ are symmetric. 


\subsection{Conformal Invariance}

The contributions $S_{0}$ and $S_{1}$ to the action are conformally invariant at the classical level. Now we wish to show that also the quantum theory is conformally invariant. We already know from section 7 that this is true for $S_{0}$ alone; we wish to show that incorporation of the ghost couplings does not spoil conformal invariance.

The interactions in $S_{1}$ are actually sigma model fields of dimension one, multiplying $\partial \phi$ or $\bar{\partial} \phi$, as we have recalled in eqn. (8.60). The trace of the stress tensor vanishes if the "soft" interactions in $S_{1}$ are turned off. Hence, with $S_{1}$ included, this trace - which we denote $T$ - is a sum of terms each of which is a sigma model operator of dimension one or zero multiplying one or two derivatives of $\phi$ and $\bar{\phi}$ and powers of $e^{\phi}$ and $e^{\bar{\phi}}$. In addition, $T$ is invariant under left and right supersymmetry. There is no left and right-invariant sigma model operator of dimension less than two except the identity, so $T$ is of the form

$$
T=\partial_{z} \phi \partial \bar{z} \bar{\phi} N\left(e^{\phi}, e^{\bar{\phi}}\right)
$$

Moreover, by familiar arguments, $N$ is divisible by $e^{\phi}$ and $e^{\bar{\phi}}$. It follows that

$$
T=\partial_{z} \partial_{\bar{z}} \widetilde{N}\left(e^{\phi}, e^{\bar{\phi}}\right)
$$

for some $\tilde{N}$; we have used the fact that for any $a, b>0, \partial_{z} \phi \partial_{\bar{z}} \bar{\phi} e^{a \phi+b \bar{\phi}}=\partial_{z} \partial_{\bar{z}} e^{a \phi+b \bar{\phi}} / a b$. But a $c$-number trace of the stress tensor of the form in (8.67) can be canceled by adding to the Lagrangian a ghost coupling to background curvature, $\int d^{2} \sigma \widetilde{N}\left(e^{\phi}, e^{\bar{\phi}}\right) R$, with $R$ the curvature scalar of the worldsheet. Upon adding such a coupling, we achieve conformal invariance. We do not know whether with a natural regularization such an addition to the action is actually needed.

\section{9. $N=2$ Constraints for $\mathbf{A d S}_{3} \times \mathbf{S}^{3}$}

In this section, we shall first define the $N=2$ superconformal constraints associated with the $\mathrm{AdS}_{3} \times \mathbf{S}^{3}$ with ghosts that was described in section 8 . The $N=4$ topological constraints can be easily constructed from these $N=2$ constraints using the method described in section 2 . We shall then show that these $N=2$ constraints commute with the ghostdependent $S U^{\prime}(2 \mid 2) \times S U^{\prime}(2 \mid 2)$ transformations found in section 8 . Using the formalism of sections 2-5, this implies that scattering amplitudes as well as physical state conditions are $S U^{\prime}(2 \mid 2) \times S U^{\prime}(2 \mid 2)$ invariant. Finally, we shall show that the $N=2$ constraints are also 
holomorphic. The proofs of $S U^{\prime}(2 \mid 2) \times S U^{\prime}(2 \mid 2)$ invariance and holomorphicity are not as precise as the rest of this paper, because we treat some aspects of the $S U^{\prime}(2 \mid 2)$ current operator products classically, potentially overlooking terms analogous to normal-ordering contributions in free field theory. We expect that a more complete treatment would be somewhat similar to the proof of $S U^{\prime}(2 \mid 2)$ invariance and conformal invariance of ghost couplings in the last section.

We have not explicitly checked that our constraints have the standard $N=2$ OPE's, which would be a useful thing to check. Probably the only difficult OPE to check would be that $G^{+}$has no singularity with itself.

\subsection{Review of Constraints in Minkowski Background}

Since the proof of $S U^{\prime}(2 \mid 2) \times S U^{\prime}(2 \mid 2)$ invariance is somewhat complicated, it will be helpful to first review this invariance for the flat Minkowski case. Recall that the $N=2$ superconformal constraints in a flat $\delta=6$ Minkowski background are given by

$$
\begin{gathered}
T=\frac{1}{8} \epsilon_{a b c d} \partial x^{a b} \partial x^{c d}+p_{a} \partial \theta^{a}+\frac{1}{2} \partial \rho \partial \rho+\frac{1}{2} \partial \sigma \partial \sigma+\frac{3}{2} \partial^{2}(\rho+i \sigma)+T_{C}^{G S} \\
G^{+}=-\frac{1}{24} e^{-2 \rho-i \sigma} \epsilon^{a b c d} p_{a} p_{b} p_{c} p_{d}+\frac{i}{2} e^{-\rho} p_{a} p_{b} \partial x^{a b}+ \\
e^{i \sigma}\left(\frac{1}{8} \epsilon_{a b c d} \partial x^{a b} \partial x^{c d}+p_{a} \partial \theta^{a}\right)+\frac{1}{2} e^{i \sigma}\left(\partial(\rho+i \sigma) \partial(\rho+i \sigma)-\partial^{2}(\rho+i \sigma)\right)+G_{C}^{+G S} \\
G^{-}=e^{-i \sigma}+G_{C}^{-G S} \\
J=\partial(\rho+i \sigma)+J_{C}^{G S} .
\end{gathered}
$$

Holomorphicity of these constraints is clear. The only non-trivial $S U^{\prime}(2 \mid 2) \times S U^{\prime}(2 \mid 2)$ invariance to check is the supersymmetry corresponding to $q_{a}^{+}=\oint\left(e^{\phi} p_{a}-\frac{i}{2} \epsilon_{a b c d} \theta^{b} \partial x^{c d}\right)$, which generates the transformation

$$
\begin{gathered}
\delta \theta^{a}=u^{a} e^{\phi}, \quad \delta p_{a}=\frac{i}{2} \epsilon_{a b c d} u^{b} \partial x^{c d}, \quad \delta x^{a b}=i\left(u^{a} \theta^{b}-u^{b} \theta^{a}\right), \\
\delta \partial(\rho-i \sigma)=2 u^{a} p_{a} e^{\phi}, \quad \delta \phi=0, \\
\delta e^{m \rho+i(m+1) \sigma}=e^{(m-1) \rho+i m \sigma} u^{a} p_{a}, \quad \delta e^{-i \sigma}=0 .
\end{gathered}
$$

Note that these transformations can be obtained by taking the contour integral of the susy generator around the worldsheet field. We recall that $\phi=-\rho-i \sigma$ and that $\rho$ and $\sigma$ have canonical OPE's. 
Under the transformation of (9.2), it is trivial to show that $\delta J=\delta G^{-}=0$. To show that $\delta T=0$, note that the transformation of $x^{a b}$ cancels the transformation of $p_{a}$ and the transformation of $\theta^{a}$ cancels the transformation of $\partial(\rho-i \sigma)$. Invariance of $G^{+}$is more complicated and inolves cancellation between various terms. The variation of $e^{-2 \rho-i \sigma}$ in the first term does not contribute since $(p)^{5}=0$. The variation of $p_{a}$ in the first term contributes

$$
\frac{i}{12} e^{-2 \rho-i \sigma} \epsilon^{a b c d} p_{b} p_{c} p_{d} \epsilon_{a e f g} u^{e} \partial x^{f g}=-\frac{i}{2} e^{-2 \rho-i \sigma} u^{a} p_{a}\left(p_{c} p_{d} \partial x^{c d}\right)
$$

which cancels the variation of $e^{-\rho}$ in the second term. The variation of $p_{a}$ and $x^{a b}$ in the second term contributes

$$
\frac{i}{2} e^{-\rho}\left(i \epsilon_{a b c d} u^{b} \partial x^{c d} p_{e} \partial x^{a e}+2 i u^{a} p_{a} p_{b} \partial \theta^{b}\right)=-e^{-\rho}\left(u^{e} p_{e}\right)\left(\frac{1}{8} \epsilon_{a b c d} \partial x^{a b} \partial x^{c d}+p_{b} \partial \theta^{b}\right)
$$

which is cancelled by the variation of $e^{i \sigma}$ in the third term of $G^{+}$. Finally, the variation of $x, \theta^{a}$ and $p_{a}$ in the third term of $G^{+}$contributes

$$
e^{i \sigma}\left(\frac{i}{2} \epsilon_{a b c d} \partial x^{a b} u^{c} \partial \theta^{d}-\frac{i}{2} \epsilon_{a b c d} u^{b} \partial x^{c d} \partial \theta^{a}+p_{a} u^{a} \partial e^{\phi}\right)=-e^{i \sigma} u^{a} p_{a} \partial e^{\phi},
$$

which is cancelled by the variation of the fourth term in $G^{+}$, namely $\frac{1}{2} e^{i \sigma}\left((\partial \phi)^{2}+\partial^{2} \phi\right)$. The easiest way to compute the variation of this fourth term is to write it as $\partial e^{\phi} e^{2 i \sigma+\rho}$, which is regularized as

$$
\partial e^{\phi} e^{2 i \sigma+\rho}=\frac{1}{2 \pi i} \oint d y \frac{\partial_{y} e^{\phi(y)}}{y-z} e^{2 i \sigma(z)+\rho(z)}
$$

where the contour integration of $y$ goes around the point $z$. To reproduce $\frac{1}{2} e^{i \sigma}\left((\partial \phi)^{2}+\partial^{2} \phi\right)$ from (9.3), one uses that

$$
\partial_{y} e^{\phi(y)} e^{2 i \sigma(z)+\rho(z)}=e^{i \sigma(z)} \partial_{y}\left[(y-z)^{-1}+\partial \phi(z)+\frac{y-z}{2}\left((\partial \phi)^{2}+\partial^{2} \phi\right)\right] .
$$

\section{2. $S U^{\prime}(2 \mid 2) \times S U^{\prime}(2 \mid 2)$ Invariance of Constraints}

In the $\mathrm{AdS}_{3} \times \mathbf{S}^{3}$ background with Ramond-Ramond coupling, we learned in the last section that the sixteen spacetime-supersymmetry transformations are now generated by the eight fermionic components of the left-invariant currents $g^{-1} d g$, and by the eight currents

$$
v_{1}^{+} E^{a}+v_{2}^{+} F^{a}, \quad v_{1}^{-} E^{a}+v_{2}^{-} F^{a}
$$


where $E^{a}$ and $F^{a}$ are the fermionic components of the right-invariant currents $d g g^{-1}$, and

$$
V=\left(\begin{array}{ll}
v_{1}^{+} & v_{2}^{+} \\
v_{1}^{-} & v_{2}^{-}
\end{array}\right)=\left(1-\frac{1}{4} e^{\phi+\bar{\phi}}\right)^{-1}\left(\begin{array}{cc}
1+\frac{1}{4} e^{\phi+\bar{\phi}} & i e^{\phi} \\
-i e^{\bar{\phi}} & 1+\frac{1}{4} e^{\phi+\bar{\phi}}
\end{array}\right)
$$

It will now be argued that the following $N=2$ generators are invariant under all $S U^{\prime}(2 \mid 2) \times S U^{\prime}(2 \mid 2)$ transformations of the action (including the $\phi$-dependent transformations of $(9.4))$ :

$$
\begin{gathered}
T=\frac{1}{8} \epsilon^{a b c d} K_{a b} K_{c d}-\frac{1}{2} \epsilon_{\alpha \beta} S^{a \alpha} S^{a \beta}+\frac{1}{2} \partial \rho \partial \rho+\frac{1}{2} \partial \sigma \partial \sigma+\frac{3}{2} \partial^{2}(\rho+i \sigma)+T_{C}^{G S} \\
G^{+}=-\frac{1}{6} e^{-2 \rho-i \sigma} \epsilon^{a b c d} U_{a b} U_{c d}+i e^{-\rho} K^{a b} U_{a b}+ \\
e^{i \sigma}\left(\frac{1}{8} \epsilon^{a b c d} K_{a b} K_{c d}-\frac{1}{2} \epsilon_{\alpha \beta} S^{a \alpha} S^{a \beta}\right)+\frac{1}{2} e^{i \sigma}\left(\partial(\rho+i \sigma) \partial(\rho+i \sigma)-\partial^{2}(\rho+i \sigma)\right)+G_{C}^{+} G S \\
G^{-}=e^{-i \sigma}+G_{C}^{-G S} \\
J=\partial(\rho+i \sigma)+J_{C}^{G S},
\end{gathered}
$$

where

$$
\begin{gathered}
U_{a b}=S_{a}^{\alpha} S_{b}^{\beta}\left(\partial c_{\alpha \beta} / \partial e^{\phi}\right)=S_{a}^{\alpha} S_{b}^{\beta} \partial c_{\alpha \beta}\left(\partial e^{\phi}\right)^{-1} \\
=\left(1-\frac{1}{4} e^{\phi+\bar{\phi}}\right)^{-2}\left[\frac{1}{2} F_{a} F_{b}-\frac{1}{8} e^{2 \bar{\phi}} E_{a} E_{b}-\frac{i}{4} e^{\bar{\phi}}\left(E_{a} F_{b}+F_{a} E_{b}\right)\right],
\end{gathered}
$$

$c_{\alpha \beta}=\epsilon_{\alpha \gamma} \epsilon_{\beta \delta} c^{\gamma \delta}$ where $c_{\gamma \delta}$ is defined in (8.38), $S_{a}^{1}=E_{a}, S_{a}^{2}=F_{a}$, and $\left[E_{a}, F_{a}, K_{a b}\right]$ are the fermionic and bosonic $z$-components of the right-invariant currents $\partial_{z} g g^{-1}$. Note that since the $\phi$-dependent part of the action is anti-symmetric in $z$ and $\bar{z}$, it does not contribute to $T$. Also note that when $E^{a}=\partial \theta^{a}, F^{a}=p^{a}, K^{a b}=\partial x^{a b}$, and $e^{\bar{\phi}}$ is set to zero, one recovers the $N=2$ constraints of (9.1) in a flat Minkowski background. The anti-holomorphic $N=2$ generators, $\left[\bar{T}, \bar{G}^{+}, \bar{G}^{-}, \bar{J}\right]$, are similar to those of (9.6) but use the $\bar{z}$-components of the right-invariant currents, $\bar{\partial} g g^{-1}$.

Under $S U^{\prime}(2 \mid 2)$ transformations acting from the right, (9.6) is clearly invariant since it is constructed using right-invariant currents. Also, under the bosonic $S U^{\prime}(2 \mid 2)$ transformations acting from the left, (9.6) is invariant since all indices are contracted in an $\mathrm{SO}$ (4)-covariant manner. So one only needs to check that (9.6) is invariant under the $\phi$-dependent susy transformation generated by

$$
\eta_{I}^{a}\left(v_{1}^{I} E^{a}+v_{2}^{I} F^{a}\right)=\eta_{I}^{a} v_{\alpha}^{I} S^{\alpha a}
$$


where $v_{\alpha}^{I}$ are defined in (9.5). Under (9.7), the right-invariant currents transform as

$$
\delta S^{\alpha a}=\epsilon^{\alpha \beta}\left(-\frac{1}{2} \epsilon^{a b c d} \eta_{I}^{b} v_{\beta}^{I} K^{c d}-\eta_{I}^{a} \partial v_{\beta}^{I}\right), \quad \delta K^{a b}=-\eta_{I}^{a} v_{\alpha}^{I} S^{\alpha b}+\eta_{I}^{b} v_{\alpha}^{I} S^{\alpha a}
$$

where the transformations of $S^{\alpha a}$ were explained in (8.31). The compactification variables are all inert under this transformation.

One also needs to define how the chiral bosons, $\rho$ and $\sigma$, transform under the susy transformation of (9.7). Since (9.7) only involves these chiral bosons in the linear combination $\phi=-\rho-i \sigma, \rho+i \sigma$ commutes with (9.7) and is therefore invariant under the susy transformation. However, since

$$
\partial(\rho(y)-i \sigma(y)) e^{n \phi(z)} \rightarrow \frac{2 n}{y-z} e^{n \phi}
$$

$\partial(\rho(y)-i \sigma(y))$ will not be invariant but will be defined to transform as

$$
\delta(\partial(\rho-i \sigma))=2 \eta_{I}^{a} S_{a}^{\alpha}\left(\partial v_{\alpha}^{I} / \partial \phi\right)=\frac{2}{\partial \phi} \eta_{I}^{a} S_{a}^{\alpha} \partial v_{\alpha}^{I}
$$

Note that $\partial v_{\alpha}^{I}=\partial \phi\left(\partial v_{\alpha}^{I} / \partial \phi\right)$ since $\partial \bar{\phi}=0$ even in the presence of $S_{1}$. The notation in (9.10) is somewhat symbolic. We have $\partial v_{\alpha}^{I}=\partial \phi f_{\alpha}^{I}\left(e^{\phi}, e^{\bar{\phi}}\right)$ for some function $f$, and we write $f_{\alpha}^{I}$ as $\frac{1}{\partial \phi} \partial v_{\alpha}^{I}$. Other formulas below must be read likewise.

In defining the above transformation we need to justify using the free field OPE (9.9) even after the deformation of the action by coupling in the ghost fields. We can treat the ghost couplings as a perturbation and bring down terms from the action, and recall that the action only involves ghost terms of the form $e^{m \phi+k \bar{\phi}}$. We then consider the OPE of any field $A(y)$ with $e^{n \phi(z)}$. Noting that $\phi(z)$ has no singularity with the fields $\phi$ or $\bar{\phi}$ coming from the ghost couplings in the deformed action, we conclude that the deformation will not modify the singularity structure of the OPE of $A(y)$ with $e^{n \phi(z)}$, and so these are the same as the free field OPE singularity. This justifies (9.9) and the OPE's we now consider. In particular we have,

$$
e^{m \rho(y)+i(m+1) \sigma(y)} e^{n \phi(z)} \rightarrow \frac{n}{y-z} e^{(m-1) \rho(y)+i m \sigma(y)} e^{(n-1) \phi(z)},
$$

where we have taken the single pole with one of the $e^{\phi}$ 's in the term $\left(e^{\phi}(z)\right)^{n}$. So we shall define

$$
\delta\left(e^{m \rho+i(m+1) \sigma}\right)=e^{(m-1) \rho+i m \sigma} \frac{1}{\partial e^{\phi}} \eta_{I}^{a} S_{a}^{\alpha} \partial v_{\alpha}^{I} .
$$


Note that $e^{(m-1) \rho+i m \sigma}$ may have poles with $\frac{1}{\partial e^{\phi}} \partial v_{\alpha}^{I}$, so this expression may need to be normal-ordered. It might be possible to explicitly compute the contributions of such normal-ordering terms, but we have not yet done so. Finally, we will need to know the susy transformation of $e^{-i \sigma}$. Since $e^{-i \sigma}$ has no poles with $e^{n \phi}$ for $n$ positive, we shall define

$$
\delta\left(e^{-i \sigma}\right)=0
$$

Using the transformations defined above, it is now straightforward to check that the $N=2$ constraints of (9.6) are invariant under (9.7). The easiest constraints to check are $G^{-}$and $J$, which are invariant using (9.13) and the fact that $\phi$ is invariant. The next easiest constraint to check is $T$, where the variation of the the sigma model term cancels the variation of the kinetic term of the chiral bosons. Note that the sigma model term can be written as $\operatorname{Str}\left(\partial g g^{-1} \partial g g^{-1}\right)$, whose variation under (9.7) is $-\eta_{I}^{a} S_{a}^{\alpha} \partial v_{\alpha}^{I}$. The kinetic term for the chiral bosons can be written as $\frac{1}{2} \partial(\rho-i \sigma) \partial(\rho+i \sigma)$, so using (9.10), its variation is $\eta_{I}^{a} S_{a}^{\alpha} \partial v_{\alpha}^{I}$.

The hardest constraint to check is $G^{+}$. To check its invariance, it will be useful to note that

$$
\partial c^{\alpha \beta} \partial c_{\beta \gamma}=\partial c_{\alpha \beta} \partial c^{\beta \gamma}=0
$$

Using

$$
v_{\gamma}^{I} \epsilon^{\gamma \alpha} \partial c_{\alpha \beta}=\frac{i}{2} \partial v_{\beta}^{I}
$$

from equation (8.35), this implies that

$$
\partial v_{\alpha}^{I} \partial c^{\alpha \beta}=2 i \epsilon^{\gamma \delta} v_{\gamma} \partial c_{\delta \alpha} \partial c^{\alpha \beta}=0
$$

Note that

$$
G^{+}=e^{-2 \rho-i \sigma} X+e^{-\rho} Y+e^{i \sigma} Z+e^{2 i \sigma+\rho} \partial e^{\phi}
$$

where $X, Y$ and $Z$ only depend on the chiral bosons in the combinations $\phi$ and $\bar{\phi}$ and the last term comes from (9.3). Defining the supersymmetry transformation of $G^{+}$by

$$
\delta G^{+}=\delta\left(e^{-2 \rho-i \sigma}\right) X+e^{-2 \rho-i \sigma} \delta X+\delta\left(e^{-\rho}\right) Y+e^{-\rho} \delta Y+\delta\left(e^{i \sigma}\right) Z+e^{i \sigma} \delta Z+\delta\left(e^{2 i \sigma+\rho}\right) \partial e^{\phi},
$$

we shall now show that $\delta G^{+}=0$. Variation of the first term in $G^{+}$gives two contributions, one coming from the variation of $e^{-2 \rho-i \sigma}$ and the other coming from the variation of $U_{a b}$. Using (9.12), the variation of $e^{-2 \rho-i \sigma}$ gives

$$
-\frac{e^{-3 \rho-2 i \sigma}}{6 \partial e^{\phi}} \eta_{I}^{e} S_{e}^{\alpha} \partial v_{\alpha}^{I} \epsilon^{a b c d} U_{a b} U_{c d}=-\frac{e^{-3 \rho-2 i \sigma}}{6\left(\partial e^{\phi}\right)^{3}} \eta_{I}^{e} \partial v_{\alpha}^{I} \partial c_{\beta \gamma} \partial c_{\delta \tau} \epsilon^{a b c d} S_{e}^{\alpha} S_{a}^{\beta} S_{b}^{\gamma} S_{c}^{\delta} S_{d}^{\tau} .
$$


Since $S_{a}^{\alpha}$ is anti-commuting,

$$
\begin{gathered}
\epsilon^{a b c d} S_{e}^{\alpha} S_{a}^{\beta} S_{b}^{\gamma} S_{c}^{\delta} S_{d}^{\tau}=\left(\epsilon_{\kappa \pi} S_{e}^{\kappa} S_{e}^{\pi}\right)\left(\epsilon^{\alpha \beta} \epsilon^{a b c d} S_{b}^{\gamma} S_{c}^{\delta} S_{d}^{\tau}\right. \\
\left.+\epsilon^{\alpha \gamma} \epsilon^{a b c d} S_{b}^{\beta} S_{c}^{\delta} S_{d}^{\tau}+\epsilon^{\alpha \delta} \epsilon^{a b c d} S_{b}^{\beta} S_{c}^{\gamma} S_{d}^{\tau}+\epsilon^{\alpha \tau} \epsilon^{a b c d} S_{b}^{\beta} S_{c}^{\gamma} S_{d}^{\delta}\right) .
\end{gathered}
$$

but contracting the $\alpha$ index of $\partial v_{I}^{\alpha}$ with any of the indices of $\partial c^{\beta \gamma} \partial c^{\delta \tau}$ gives zero because of (9.16), so this contribution vanishes identically.

The second contribution from the first term of $G^{+}$comes from the variation of $S_{a}^{\alpha}$ and is

$$
\begin{gathered}
\frac{4 e^{-2 \rho-i \sigma}}{6 \partial e^{\phi}} \epsilon^{a b c d}\left(\frac{1}{2} \epsilon_{a e f g} \eta_{I}^{e} K^{f g} v_{\tau}^{I}+\eta_{I}^{a} \partial v_{\tau}^{I}\right) \epsilon^{\alpha \tau}\left(\partial c_{\alpha \beta}\right) S_{b}^{\beta} U_{c d} \\
=\frac{e^{-2 \rho-i \sigma}}{3 \partial e^{\phi}} v_{\tau}^{I}\left(2 \eta_{I}^{b} K^{c d}+4 \eta_{I}^{c} K^{d b}\right) \epsilon^{\alpha \tau}\left(\partial c_{\alpha \beta}\right) S_{b}^{\beta} U_{c d} \\
=-\frac{i e^{-2 \rho-i \sigma}}{6 \partial e^{\phi}} \partial v_{\beta}^{I}\left(2 \eta_{I}^{b} K^{c d}+4 \eta_{I}^{c} K^{d b}\right) S_{b}^{\beta} U_{c d}
\end{gathered}
$$

where we have used (9.15), (9.16) and that $\epsilon^{a b c d} \epsilon_{a e f g}=\delta_{e}^{b} \delta_{f}^{c} \delta_{g}^{d}$ plus cyclic permutations. The variation (9.19) can be put in a simpler form by noting that

$$
\begin{gathered}
\partial v_{\beta}^{I} S_{b}^{\beta} U_{c d}=\partial v_{\beta}^{I} S_{b}^{\beta} S_{c}^{\gamma} S_{d}^{\delta} \frac{\partial c_{\gamma \delta}}{\partial e^{\phi}} \\
=\partial v_{\beta}^{I}\left(S_{b}^{\gamma} S_{c}^{\beta}-\epsilon^{\beta \gamma}\left(\frac{1}{2} \epsilon_{\tau \kappa} S_{b}^{\tau} S_{c}^{\kappa}\right)\right) S_{d}^{\delta} \frac{\partial c_{\gamma \delta}}{\partial e^{\phi}} \\
=\partial v_{\beta}^{I} S_{b}^{\gamma} S_{c}^{\beta} S_{d}^{\delta} \frac{\partial c_{\gamma \delta}}{\partial e^{\phi}}=-\partial v_{\beta}^{I} S_{c}^{\beta} U_{b d},
\end{gathered}
$$

where we have used (9.16) to go from the second to the last line. Using (9.20), one can finally write the variation of the first term of $G^{+}$in (9.19) as

$$
-\frac{i e^{-2 \rho-i \sigma}}{\partial e^{\phi}} \eta_{I}^{b} \partial v_{\beta}^{I} S_{b}^{\beta} K^{c d} U_{c d}
$$

Using (9.12), it is easy to see that (9.21) is precisely cancelled by the variation of $e^{-\rho}$ in the second term of $G^{+}$.

One also gets contributions from varying $S_{a}^{\alpha}$ and $K_{a b}$ in the second term of $G^{+}$. Using (9.8), these contribute

$$
\begin{aligned}
& -\frac{2 i}{\partial e^{\phi}} e^{-\rho}\left(\eta_{I}^{a} S^{b \beta} v_{\beta}^{I} S_{a}^{\gamma} S_{b}^{\delta} \partial c_{\gamma \delta}+K^{a b}\left(\frac{1}{2} \epsilon_{a e f g} \eta_{I}^{e} K^{f g} v_{\tau}^{I}+\eta_{I}^{a} \partial v_{\tau}^{I}\right) \epsilon^{\alpha \tau} \partial c_{\alpha \beta} S_{b}^{\beta}\right) \\
= & -\frac{2 i}{\partial e^{\phi}} e^{-\rho}\left(\eta_{I}^{a} \epsilon^{\beta \delta} v_{\beta}^{I} S_{a}^{\gamma}\left(\frac{1}{2} \epsilon_{\kappa \pi} S_{b}^{\kappa} S_{b}^{\pi}\right) \partial c_{\gamma \delta}+\frac{1}{2} \epsilon_{a e f g} \eta_{I}^{e} K^{a b} K^{f g} v_{\tau}^{I} \epsilon^{\alpha \tau}\left(\partial c_{\alpha \beta}\right) S_{b}^{\beta}\right)
\end{aligned}
$$




$$
=\frac{1}{\partial e^{\phi}} e^{-\rho}\left(\eta_{I}^{a}\left(\partial v_{\gamma}^{I}\right) S_{a}^{\gamma}\left(\frac{1}{2} \epsilon_{\kappa \pi} S_{b}^{\kappa} S_{b}^{\pi}\right)-\frac{1}{2} \epsilon_{a e f g} \eta_{I}^{e} K^{a b} K^{f g} S_{b}^{\beta} \partial v_{\beta}^{I}\right)
$$

where we have used (9.16) to go from the first to the second line and (9.15) to go from the second to the third line. Since $\epsilon_{a e f g} K^{a b} K^{f g}=\frac{1}{4} \delta_{e}^{b} \epsilon_{a c f g} K^{a c} K^{f g}$, 9.22) can be written as

$$
-\frac{1}{2 \partial e^{\phi}} e^{-\rho}\left(\eta_{I}^{a} \partial v_{\gamma}^{I} S_{a}^{\gamma}\right)\left(-\epsilon_{\kappa \pi} S_{b}^{\kappa} S_{b}^{\pi}+\frac{1}{4} \epsilon_{a c f g} K^{a c} K^{f g}\right)=-\frac{e^{-\rho}}{\partial e^{\phi}} \eta_{I}^{a} \partial v_{\gamma}^{I} S_{a}^{\gamma} \operatorname{Str}\left(\partial g g^{-1} \partial g g^{-1}\right)
$$

which is cancelled by the variation of the $e^{i \sigma}$ in the third term of $G^{+}$.

Finally, it will be shown that the remaining contribution to the variation of the third term in $G^{+}$is cancelled by the variation of the fourth term in $G^{+}$. From varying the sigma model stress-tensor, the remainining contribution to the third term in $G^{+}$is given by

$$
-\eta_{I}^{a} S_{a}^{\alpha} \partial v_{\alpha}^{I} e^{i \sigma}
$$

but by writing the fourth term in $G^{+}$as in (9.3), it is easy to see that (9.24) is cancelled by the variation of the $e^{2 i \sigma+\rho}$ in $(9.3)$. So we have shown, up to normal ordering, that the constraints of $(9.6)$ are $S U^{\prime}(2 \mid 2)$-invariant.

\subsection{Holomorphicity of Constraints}

It will now be shown that, in addition to being invariant under $S U^{\prime}(2 \mid 2) \times S U^{\prime}(2 \mid 2)$, the $N=2$ constraints of (9.6) are also holomorphic. To prove this, we will need to know some facts about the chiral bosons. The first fact is that $\bar{\partial} \phi=0$. This is because the action only involves $\rho$ and $\sigma$ in the combination $\rho+i \sigma$, so $\partial \bar{\partial}(\rho+i \sigma)=0$ even after including the $\phi$-dependent terms of $S_{1}$ into the action. However, once those $\phi$-dependent couplings are included, $\rho-i \sigma$ is no longer holomorphic. Rather, in the presence of those couplings the equation of motion for $\phi$ gives $\partial \bar{\partial}(\rho-i \sigma)=2 \partial S_{1} / \partial \phi$. Another useful fact is that $\bar{\partial} e^{-i \sigma}=0$. This can be seen by unbosonizing the $\sigma$ field back into $b=e^{-i \sigma}$ and $c=e^{i \sigma}$. Since the $\phi$-dependent part of the action only includes positive powers of $e^{\phi}=e^{-\rho-i \sigma}$, it contains $b$ dependence but not $c$ dependence. So the equation of motion for $c$ is still $\bar{\partial} b=0$. A final useful fact is that

$$
\bar{\partial} e^{i(m+1) \sigma+m \rho}=-e^{i m \sigma+(m-1) \rho} \frac{i}{\partial e^{\phi}} \bar{S}_{a}^{\alpha} \partial c_{\alpha \beta} S_{a}^{\beta}
$$

where $\bar{S}_{a}^{\beta}$ is defined to be the $\bar{z}$ component of the left-supersymmetry current $S_{a}^{\alpha}$. Note that when there is no bar over the current, it will be assumed to be the $z$ component of 
the current. Equation (9.25) can be heuristically justified in a manner similar to (9.12), but there may be normal-ordering contributions that have not been included.

To prove holomorphicity of the constraints, we will also need to know what are $\bar{\partial} K_{a b}$ and $\bar{\partial} S_{a}^{\alpha}$. Since the bosonic $S U^{\prime}(2 \mid 2) \times S U^{\prime}(2 \mid 2)$ transformations are unmodified in the presence of $S_{1}, K_{a b}$ is conserved on-shell, i.e. $\bar{\partial} K_{a b}=-\partial \bar{K}_{a b}$. The Maurer-Cartan equation implies that

$$
\partial \bar{K}_{a b}-\bar{\partial} K_{a b}=-\epsilon_{\alpha \beta}\left(S_{a}^{\alpha} \bar{S}_{b}^{\beta}-\bar{S}_{a}^{\alpha} S_{b}^{\beta}\right)+\epsilon_{a b c d} K^{c e} \bar{K}^{d e}
$$

SO

$$
\bar{\partial} K_{a b}=\frac{1}{2} \epsilon_{\alpha \beta}\left(S_{a}^{\alpha} \bar{S}_{b}^{\beta}-\bar{S}_{a}^{\alpha} S_{b}^{\beta}\right)-\frac{1}{2} \epsilon_{a b c d} K^{c e} \bar{K}^{d e} .
$$

In the presence of $S_{1}$, the conserved supersymmetries are given by $v_{\alpha}^{I} S_{a}^{\alpha}$, so $\bar{\partial}\left(v_{\alpha}^{I} S_{a}^{\alpha}\right)=$ $-\partial\left(v_{\alpha}^{I} \bar{S}_{a}^{\alpha}\right)$ where $v_{\alpha}^{I}$ is defined in (9.5). Using (9.15), this implies that

$$
\bar{\partial} S_{a}^{\delta}+2 i \epsilon_{\alpha \beta}\left(\bar{\partial} c^{\beta \delta}\right) S_{a}^{\alpha}=-\partial \bar{S}_{a}^{I}+2 i \epsilon_{\alpha \beta}\left(\partial c^{\beta \delta}\right) \bar{S}_{a}^{\alpha}
$$

Using the Maurer-Cartan equations, $\partial \bar{S}_{a}^{\alpha}-\bar{\partial} S_{a}^{\alpha}=-\frac{1}{2} \epsilon_{a b c d}\left(K_{b c} \bar{S}_{d}^{\alpha}-\bar{K}_{b c} S_{d}^{\alpha}\right)$, one learns that

$$
\bar{\partial} S_{a}^{\alpha}=-i \epsilon_{\gamma \beta}\left(S_{a}^{\gamma} \bar{\partial} c^{\alpha \beta}-\bar{S}_{a}^{\gamma} \partial c^{\beta \alpha}\right)+\frac{1}{4} \epsilon_{a b c d}\left(K_{b c} \bar{S}_{d}^{\alpha}-\bar{K}_{b c} S_{d}^{\alpha}\right)
$$

Using these equations for the chiral bosons and the right-invariant currents, one is now ready to prove the holomorphicity of the constraints. That $\bar{\partial} T=0$ was proved in section 8. Also, $\bar{\partial} G^{-}=\bar{\partial} J=0$ using the above facts about chiral bosons. Once again, $\bar{\partial} G^{+}=0$ is the most difficult equation to prove, but our task will be simplified by the knowledge that $G^{+}$is invariant under left supersymmetry. Expanding $G^{+}$as in (9.17), we shall define

$$
\bar{\partial} G^{+}=\bar{\partial}\left(e^{-2 \rho-i \sigma}\right) X+e^{-2 \rho-i \sigma} \bar{\partial} X+\bar{\partial}\left(e^{-\rho}\right) Y+e^{-\rho} \bar{\partial} Y+\bar{\partial}\left(e^{i \sigma}\right) Z+e^{i \sigma} \bar{\partial} Z+\bar{\partial}\left(e^{2 i \sigma+\rho}\right) \partial e^{\phi} .
$$

First, note that if one takes the supersymmetry parameter to be

$$
\eta_{I}^{a}=-\frac{1}{2} \epsilon_{\alpha \beta}\left(v^{-1}\right)_{I}^{\alpha} \bar{S}^{\beta a}
$$

then the supersymmetric transformation law of $e^{m \rho+i(m+1) \sigma}$ becomes $\delta\left(e^{m \rho+i(m+1) \sigma}\right)=$ $\bar{\partial} e^{m \rho+i(m+1) \sigma}$ using (9.15), (9.12), and (9.25). Here we have defined $v^{-1}$ by $\left(v^{-1}\right)_{I}^{\alpha} v_{\beta}^{I}=\delta_{\beta}^{\alpha}$. Second, note that when $\eta_{I}^{\alpha}$ is chosen as in (9.29), (9.8) and (9.26) imply that

$$
\bar{\partial} K_{a b}-\delta K_{a b}=-\frac{1}{2} \epsilon_{a b c d} K^{c e} \bar{K}^{d e} .
$$


Finally, note that when $\eta_{I}^{\alpha}$ is chosen as in (9.29), (9.8) and (9.27) imply that

$$
\bar{\partial} S_{a}^{\alpha}-\delta S_{a}^{\alpha}=-i \epsilon_{\gamma \beta} S_{a}^{\gamma} \bar{\partial} c^{\alpha \beta}-\frac{1}{4} \epsilon_{a b c d} \bar{K}_{b c} S_{d}^{\alpha}
$$

Using the knowledge that $\delta G^{+}=0$, one can therefore conclude that $\bar{\partial} G^{+}$gets contributions from only three sources. The first source is from (9.30), the second source is form (9.31), and the third source is from when the $\bar{\partial}$ hits a $\bar{\partial} c_{\alpha \beta}$ in $G^{+}$. These three sources contribute only to the terms $\bar{\partial} X, \bar{\partial} Y$ and $\bar{\partial} Z$ in the computation of (9.28). All other contributions cancel out using the proof of the previous subsection. It will now be shown that the contributions from these three sources cancel each other out in $\bar{\partial} X, \bar{\partial} Y$, and $\bar{\partial} Z$, implying that $\bar{\partial} G^{+}=0$.

First, consider the contribution of (9.31) to $\bar{\partial} X$, which is

$$
\frac{2}{3\left(\partial e^{\phi}\right)^{2}} \epsilon^{a b c d}\left(i \epsilon_{\gamma \beta} S_{a}^{\gamma} \bar{\partial} c^{\alpha \beta}+\frac{1}{4} \epsilon_{a e f g} \bar{K}_{e f} S_{g}^{\alpha}\right) \partial c_{\alpha \delta} S_{b}^{\delta} S_{c}^{\tau} \partial c_{\tau \kappa} S_{d}^{\kappa}
$$

The term involving $\bar{K}_{e f}$ is zero since, after writing $\epsilon^{a b c d} \epsilon_{a e f g}$ as $\delta_{e}^{b} \delta_{f}^{c} \delta_{g}^{d}$ plus cyclic permutations, one can use $S_{a}^{\alpha} \partial c_{\alpha \beta} S_{a}^{\beta}=0$ since $c_{\alpha \beta}$ is a symmetric matrix, and

$$
S_{c}^{\alpha} \partial c_{\alpha \delta} S_{c}^{\tau} \partial c_{\tau \kappa}=-\frac{1}{2} \epsilon^{\alpha \tau}\left(\epsilon_{\pi \sigma} S_{c}^{\pi} S_{c}^{\sigma}\right) \partial c_{\alpha \delta} \partial c_{\tau \kappa}=0
$$

using (9.14). The remaining term is

$$
\begin{gathered}
\frac{2 i}{3\left(\partial e^{\phi}\right)^{2}} \epsilon^{a b c d} \epsilon_{\gamma \beta} S_{a}^{\gamma} \bar{\partial} c^{\beta \alpha} \partial c_{\alpha \delta} S_{b}^{\delta} S_{c}^{\tau} \partial c_{\tau \kappa} S_{d}^{\kappa} \\
=\frac{1}{3\left(\partial e^{\phi}\right)^{2}} \epsilon^{a b c d} S_{a}^{\gamma} \partial \bar{\partial} c_{\gamma \delta} S_{b}^{\delta} S_{c}^{\tau} \partial c_{\tau \kappa} S_{d}^{\kappa}
\end{gathered}
$$

since (8.37) implies that

$$
\bar{\partial} \partial c_{\gamma \delta}=i\left(\epsilon_{\gamma \beta} \bar{\partial} c^{\beta \alpha} \partial c_{\alpha \delta}+\epsilon_{\delta \beta} \bar{\partial} c^{\beta \alpha} \partial c_{\alpha \gamma}\right)
$$

But (9.32) is cancelled by the contribution when the $\bar{\partial}$ hits the $c_{\alpha \beta}$ 's, so the sources cancel out in the $\bar{\partial} X$ term.

Next, consider the contribution of (9.31) to $\bar{\partial} Y$, which is

$$
-\frac{2 i}{\partial e^{\phi}}\left(i \epsilon_{\gamma \beta} S_{a}^{\gamma} \bar{\partial} c^{\alpha \beta}+\frac{1}{4} \epsilon_{a e f g} \bar{K}_{e f} S_{g}^{\alpha}\right) \partial c_{\alpha \delta} S_{b}^{\delta} K^{a b} .
$$


The term involving $\bar{K}_{e f}$ in (9.33) is cancelled by the contribution of (9.30) to $\bar{\partial} Y$, which is

$$
-\frac{i}{2} U^{a b} \epsilon_{a b c d} K^{c e} \bar{K}^{d e}=\frac{i}{2} \epsilon_{a e f g} \bar{K}^{e f} K^{a b} U^{g b} .
$$

The remaining term in $(9.33)$ is

$$
\frac{2}{\partial e^{\phi}} \epsilon_{\gamma \beta} S_{a}^{\gamma} \bar{\partial} c^{\beta \alpha} \partial c_{\alpha \delta} S_{b}^{\delta} K^{a b}=-\frac{i}{\partial e^{\phi}} S_{a}^{\gamma} \bar{\partial} \partial c_{\gamma \delta} S_{b}^{\delta} K^{a b},
$$

which is cancelled by the contribution to $\bar{\partial} Y$ when the $\bar{\partial}$ hits the $c_{\alpha \beta}$.

Finally, consider the contribution of (9.31) to $\bar{\partial} Z$, which is

$$
\epsilon_{\alpha \beta}\left(i \epsilon_{\gamma \delta} S_{a}^{\gamma} \bar{\partial} c^{\alpha \delta}+\frac{1}{4} \epsilon_{a e f g} \bar{K}_{e f} S_{g}^{\alpha}\right) S_{a}^{\beta} .
$$

But this vanishes since $S_{a}^{\alpha}$ is anti-commuting. Similarly, the contribution of (9.30) to $\bar{\partial} Z$ vanishes since

$$
\epsilon_{a b c d} K^{a b} \epsilon_{c d e f} K^{e g} \bar{K}^{f g}=4 K^{e f} K^{e g} \bar{K}^{f g}=0
$$

by symmetry in $f$ and $g$ of $K^{e f} K^{e g}$. Since there are no $c_{\alpha \beta}$ terms in $Z$, we have proven (up to normal-ordering) that $\bar{\partial} G^{+}=0$.

\section{WZW on $S U^{\prime}(2 \mid 2)$}

It would be nice to be able to completely solve the two parameter conformal theory we have defined above. One of the two parameters, $k$ is quantized and so is not associated with a marginal deformation of the theory, whereas the other parameter, $1 / f^{2}$, controls the radius of the target and is associated with such a deformation. The experience with conformal theories suggests that finding an exactly solvable conformal theory along a whole line of marginal deformations is extremely rare. In fact, the only known examples involve tori and their orbifolds. It is not clear to us whether one should expect in the case at hand an exactly solvable theory for all $1 / f^{2}$ and fixed $k$. However there is one point at which this must be so and that is the WZW limit where $1 / f^{2}=|k|$. In this limit we should get an affine Kac-Moody algebra based on the supergroup $S U^{\prime}(2 \mid 2)$ at level $k$, which should be soluble to a large extent. The $S U^{\prime}(2 \mid 2)$ current algebra can be written explicitly as follows:

$$
K_{a b}(z) K_{c d}(0) \sim \frac{k \epsilon^{a b c d}}{z^{2}}+\frac{\delta_{a c} K_{b d}(0)-\delta_{a d} K_{b c}(0)-\delta_{b c} K_{a d}(0)+\delta_{b d} K_{a c}(0)}{z}
$$




$$
\begin{gathered}
S_{a \alpha}(z) S_{b \beta}(0) \sim \frac{k \delta_{a b} \epsilon_{\alpha \beta}}{z^{2}}+\frac{\frac{1}{2} \epsilon_{\alpha \beta} \epsilon_{a b c d} K^{c d}(0)}{z} \\
K_{a b}(z) S_{c \alpha}(0) \sim \frac{\delta_{a c} S_{b \alpha}(0)-\delta_{b c} S_{a \alpha}(0)}{z}
\end{gathered}
$$

We will see how this current algebra can be realized in terms of more familiar theories.20

The flat model on $\mathbf{R}^{6}$ times $\mathrm{K} 3$ or $\mathbf{T}^{4}$ was described in section 4 in the spacetime supersymmetric formalism using free fermions $\left(p_{a}, \theta^{b}\right)$ of spins $(1,0)$. From our discussion in section 6.4, we anticipate that after deformation to $\mathrm{AdS}_{3} \times \mathbf{S}^{3}$ with only NS flux, the model can be described by a WZW model of $S U^{\prime}(2 \mid 2)$ that is obtained by integrating out $p$, but can also be realized by a system in which the fermions $(p, \theta)$ are both retained and have a first order kinetic energy. We can argue more specifically that in this description, $p$ and $\theta$ are simply free fields, just as in the $\mathbf{R}^{6}$ case. In fact, in the RNS approach, the $\operatorname{AdS}_{3} \times \mathbf{S}^{3}$ background with NS flux only is described [10] by an $S U(1,1) \times S U(2)$ current algebra with free fermions. Such a current algebra is constructed from bosonic $S U(1,1) \times S U(2)$ current algebra plus free RNS fermions $\psi^{i}$. The RNS approach is mapped to the supersymmetric approach by a change of variables described in detail for the $\mathbf{R}^{6}$ case in section 4 . This change of variables maps $\psi^{i}$ plus ghosts to $p_{a}, \theta^{b}$ plus some ghost-like fields. Going from $\mathbf{R}^{6}$ to $\mathrm{AdS}_{3} \times \mathbf{S}^{3}$ should not really change that story; in the RNS description the $\psi^{i}$ are still free, so the same change of variables can be made in the same way and gives free fields $p, \theta$. Thus, we have a prediction that the current algebra of $S U^{\prime}(2 \mid 2)$ can be realized by $S U(2) \times S U(2)$ bosonic current algebra plus free fermions $p, \theta$ of spins $(1,0)$ (with a similar statement relating $S U^{\prime}(1,1 \mid 2)$ to $\left.S U(1,1) \times S U(2)\right)$. In fact, this has already been shown [23], and we will review it below.

The $S U^{\prime}(2 \mid 2)$ current algebra, restricted to $S U(2) \times S U(2)$, is at levels $(-k, k)$, where the minus sign for one of the $S U(2)$ 's is familiar from section 7 . Since inclusion of $p, \theta$ will shift the level by +2 for each $S U(2)$, we must start with a purely bosonic current algebra at level $(-k-2, k-2)$ for the two $S U(2)$ 's:

$$
c=\frac{3(k-2)}{(k-2)+2}+\frac{3(-k-2)}{(-k-2)+2}=6 .
$$

20 It would be interesting to revisit sigma models on super Calabi-Yau manifolds proposed in 22] as mirror to rigid Calabi-Yau manifolds, in light of the observation here that sigma model on supermanifolds may in some cases be equivalent to more familiar systems. 
What central charge do we expect for the $S U^{\prime}(2 \mid 2)$ current algebra? For any group at level $k$, the central charge is

$$
c=\frac{k \operatorname{dim} \mathrm{G}}{k+c_{2}(G)} .
$$

Here $c_{2}(G)$ denotes the dual coxeter number of the group $G$, and in the case of a supergroup, $\operatorname{dim} G$ is interpreted as the super-dimension of the group. For $S U^{\prime}(2 \mid 2)$, as we know from section $7, c_{2}(G)=0$, so the above formula becomes $c=\operatorname{dim} G$. The superdimension of $S U^{\prime}(2 \mid 2)$ is -2 , so we expect $c=-2$. This agrees with the contribution 6 from the bosonic current algebra plus $4(-2)=-8$ from four pairs of spin $(1,0)$ free fermions.

We will now complete the dictionary between the currents $K^{a b}$ and $S_{\alpha}^{a}$ of $S U^{\prime}(2 \mid 2)$ and the bosonic currents $j^{a b}$ of $S U(2) \times S U(2)$ of WZW and the free fermions $\left(p_{a}, \theta^{a}\right)$. (In writing the bosonic currents as $j^{a b}$, we identify the adjoint representation of $S U(2) \times$ $S U(2)=S O(4)$ with the antisymmetric tensor representation of $S O(4)$.) The $S O(4) \mathrm{OPE}$ structure of the $S O(4)$ currents together with $p$ and $\theta$ is given (for the left-movers) by 21

$$
\begin{gathered}
j_{a b}(z) j_{c d}(0) \sim \frac{k \epsilon_{a b c d}+2 \delta_{a c} \delta_{b d}-2 \delta_{a d} \delta_{b c}}{z^{2}}+\frac{\delta_{a c} j_{b d}(0)-\delta_{a d} j_{b c}(0)-\delta_{b c} j_{a d}(0)+\delta_{b d} j_{a c}(0)}{z} \\
p_{a}(z) \theta_{b}(0) \sim \frac{\delta_{a b}}{z} \\
p_{a}(z) j_{b c}(0) \sim 0 \quad \theta_{a}(z) j_{b c}(0) \sim 0
\end{gathered}
$$

The $S U^{\prime}(2 \mid 2)$ currents can be constructed out of these fields as follows:

$$
\begin{gathered}
K^{a b}(z)=j^{a b}(z)-\left(p^{a}(z) \theta^{b}(z)-p^{b}(z) \theta^{a}(z)\right) \\
S_{a}^{1}(z)=k \partial \theta_{a}(z)+\frac{1}{2} \epsilon_{a b c d} \theta^{b}(z)\left(j^{c d}(z)+\theta^{c}(z) p^{d}(z)\right) \\
S_{a}^{2}(z)=p_{a}(z)
\end{gathered}
$$

It is straightforward to check that these currents satisfy the expected WZW OPE's for the affine Kac-Moody $S U^{\prime}(2 \mid 2)$ given above. As explained in (8.51) and (8.52), the left and right spacetime-supersymmetry generators are related to $S_{a}^{\alpha}$ and $\bar{S}_{a}^{\alpha}$ by

$$
q_{a}^{+}=-i\left(S_{a}^{1}+i e^{\phi} S_{a}^{2}\right), \quad q_{a}^{-}=S_{a}^{2},
$$

21 If the levels of the $S U(2)$ 's were equal and opposite, the double pole in the $j_{a b} \cdot j_{c d}$ operator product would be proportional to $\epsilon_{a b c d}$. Because of the shifts $(-k, k) \rightarrow(-k+2, k+2)$, this is not quite so and there is a term proportional to $\delta_{a c} \delta_{b d}-\delta_{a d} \delta_{b c}$. 


$$
\bar{q}_{a}^{+}=-i\left(\bar{S}_{a}^{1}+i e^{\bar{\phi}} \bar{S}_{a}^{2}\right), \quad \bar{q}_{a}^{-}=\bar{S}_{a}^{2} .
$$

For application to string theory, we also must describe the global $N=4$ algebra, introduced for $\mathbf{R}^{6}$ times K3 or $\mathbf{T}^{4}$ in section 4 . To replace $\mathbf{R}^{6}$ by $\operatorname{AdS}_{3} \times \mathbf{S}^{3}$, we make a relatively simple modification using the above-described fields. Thus, we write the $N=4$ algebra as follows:

$$
\begin{gathered}
T=T_{S U^{\prime}(2 \mid 2)}+\frac{1}{2} \partial \rho \partial \rho+\frac{1}{2} \partial \sigma \partial \sigma+\frac{3}{2} \partial^{2}(\rho+i \sigma)+T_{C}^{G S} \\
G^{+}=-e^{-2 \rho-i \sigma}(p)^{4}+\frac{i}{2} e^{-\rho}\left(p_{a} p_{b} K^{a b}+\frac{p_{a} \partial p_{a}}{2 k}\right) \\
+e^{i \sigma}\left(T_{S U^{\prime}(2 \mid 2)}+\frac{1}{2} \partial(\rho+i \sigma) \partial(\rho+i \sigma)-\frac{1}{2} \partial^{2}(\rho+i \sigma)\right)+G_{C}^{+G S} \\
G^{-}=e^{-i \sigma}+G_{C}^{-G S} \\
J=\partial(\rho+i \sigma)+J_{C}^{G S} \\
\widetilde{G}^{+}=e^{J_{C}^{G S}+\rho}+e^{\rho+i \sigma} \widetilde{G}_{C}^{+} G S \\
\widetilde{G}^{-}=e^{-J_{C}^{G S}}\left(-e^{-3 \rho-2 i \sigma}(p)^{4}+\frac{i}{2} e^{-2 \rho-i \sigma}\left(p_{a} p_{b} K^{a b}+\frac{p_{a} \partial p_{a}}{2 k}\right)\right. \\
\left.+e^{-\rho}\left(T_{S U^{\prime}(2 \mid 2)}+\frac{1}{2} \partial(\rho+i \sigma) \partial(\rho+i \sigma)-\frac{1}{2} \partial^{2}(\rho+i \sigma)\right)\right)+e^{-\rho-i \sigma} \widetilde{G}_{C}^{-} G S \\
J^{++}=e^{\rho+i \sigma} J_{C}^{++} G S \\
J^{--}=e^{-\rho-i \sigma} J_{C}^{--G S}
\end{gathered}
$$

where $T_{S U^{\prime}(2 \mid 2)}$ is the stress tensor for the $S U^{\prime}(2 \mid 2)$ WZW model

$$
T_{S U^{\prime}(2 \mid 2)}=\frac{\epsilon_{a b c d} K^{a b} K^{c d}}{8 k}+\frac{\epsilon_{\alpha \beta} S^{a \alpha} S^{a \beta}}{2 k} .
$$

Most of these formulas are obtained from the $\mathbf{R}^{6}$ case by replacing $d x$ by $K$ in the obvious way; however, the terms involving $p \partial p / 2 k$ in $G^{+}$and $\widetilde{G}^{-}$are new. These terms arise from a multiple contraction in the current algebra. The verification of supersymmetry and holomorphy of the constraints is analogous to the flat space case reviewed at the beginning of section 9 .

Acknowledgements: We would like to thank M. Bershadsky, L. Dolan, P. Etingof, V. Kac, G. Sotkov and A. Strominger for valuable discussions. NB would also like to thank Harvard University, the Institute for Advanced Study, and Rutgers University for their hospitality, and would like to thank CNPq grant 300256/94-9 and FAPESP grant 98/04086-5 for partial financial support. Research of CV was supported in part by NSF grant PHY-98-02709. Research of EW was partly supported by NSF Grant PHY-9513835. 


\section{References}

[1] D. Friedan, E. Martinec, and S. Shenker, "Conformal Invariance, Supersymmetry, and String Theory," Nucl. Phys. B271 (1986) 93.

[2] M. B. Green and J. H. Schwarz, "Covariant Description Of Superstrings," Phys. Lett. 136B (1984) 367.

[3] N. Berkovits, "Covariant Quantization Of The Green-Schwarz Superstring In A Calabi-Yau Background," Nucl. Phys. B431 (1994) 258, "A New Description Of The Superstring," Jorge Swieca Summer School 1995, p. 490, hep-th/9604123.

[4] N. Berkovits and C. Vafa, $N=4$ Topological Strings, Nucl. Phys. B433 (1995) 123, hep-th/9407190.

[5] N. Berkovits, "Quantization of the Superstring with Manifest U(5) Super-Poincaré Invariance", hep-th/99020??.

[6] N. Berkovits and W. Siegel, "Superspace Effective Actions For 4-D Compactifications Of Heterotic And Type II Superstrings," Nucl. Phys. B462 (1996) 213.

[7] J. de Boer and K. Skenderis, "Covariant Computation of the Low Energy Effective Action of the Heterotic Superstring", Nucl. Phys. B481 (1996) 129.

[8] J. Maldacena, "The Large $N$ Limit Of Superconformal Field Theories And Supergravity," Adv. Theor. Math. Phys. 2 (1998) 231.

[9] J. Maldacena and A. Strominger, " $\mathrm{AdS}_{3}$ Black Holes And A Stringy Exclusion Principle," hep-th/9804085.

[10] A. Giveon, D. Kutasov, and N. Seiberg, "Comments On String Theory On AdS 3 ," hep-th/9806194.

[11] J. de Boer, H. Ooguri, H. Robins, and J. Tannenhauser, "String Theory On AdS 3 ," hep-th/9812046.

[12] J. Balog, L. O'Raifeartaigh, P. Forgacs and A. Wipf, "Consistency of String Propagation on Curved Space-Times: An SU(1,1) Based Counterexample", Nucl. Phys. B325 (1989) 225;

P.M.S. Petropoulos, "Comments on SU(1,1) String Theory", Phys. Lett. B236 (1990) 151 ;

I. Bars and D. Nemeschansky, "String Propagation In Backgrounds With Curved Space-Time," Nucl. Phys. B348 (1991) 89 ;

M. Henningson, S. Hwang, P. Roberts, and B. Sundborg, "Modular Invariance Of SU (1, 1) Strings," Phys. Lett. B267 (1991) 350 ;

S. Hwang, "No-Ghost Theorem for SU(1,1) String Theories", Nucl. Phys. B354 (1991) 100 ;

I. Bars, "Ghost-Free Spectrum Of A Quantum String In $S L(2, \mathbf{R})$ Curved Space-Time, Phys. Rev. D53 (1996) 3308, hep-th/9503205, "Solution Of The $S L(2, \mathbf{R})$ String In 
Curved Spacetime," in Future Perspectives In String Theory (Los Angeles, 1995), hepth/9511187;

J. Teschner, "The Minisuperspace Limit Of The $S L(2, \mathbf{C}) / S U(2)$ WZNW Model," hep-th/9712258;

J. M. Evans, M. R. Geberdiel, and M. J. Perry, "The No-Ghost Theorem And Strings On $\mathrm{AdS}_{3}, "$ hep-th/9812252.

[13] R. R. Metsaev and A. A. Tseytlin, "Type IIB Superstring Action In $\mathrm{AdS}_{5} \times \mathbf{S}^{5}$ Background," Nucl. Phys. B533 (1998) 109, hep-th/9805028;

R. Kallosh, J. Rahmfeld, and A. Rajaraman, "Near Horizon Superspace," hepth/9805217;

R. Kallosh and J. Rahmfeld, "The GS String Action On $\operatorname{AdS}_{5} \times \mathbf{S}^{5}$," Phys. Lett. B443 (1998) 143, hep-th/9808038;

I. Pesando, "A k Gauge Fixed Type IIB Superstring Action On $\mathrm{AdS}_{5} \times \mathbf{S}^{5}$, JHEP 11 (1998) 002, hep-th/9808020;

I. Pesando, "The GS Type IIB Superstring Action On $\mathrm{AdS}_{3} \times \mathbf{S}^{3} \times \mathbf{T}^{4}$, hep-th/9809145;

J. Rahmfeld and A. Rajaraman, "The GS String Action On $\mathrm{AdS}_{3} \times \mathbf{S}^{3}$ with RamondRamond Charge," hep-th/9809164 ;

J. Park and S.-J. Rey, "Green-Schwarz Superstring On $\mathrm{AdS}_{3} \times \mathbf{S}^{3}$, hep-th/9812062.

[14] M. Yu and B. Zhang, "Light-Cone Gauge Quantization Of String Theories On $\mathrm{AdS}_{3}$ Space," hep-th/9812216;

A. Rajaraman and M. Rozali, "On the Quantization of the GS String on $\operatorname{AdS}_{5} \times \mathbf{S}^{5}$ ", hep-th/9902046.

[15] E. Witten, "On the Structure of the Topological Phase of Two-Dimensional Gravity," Nucl. Phys. B340 (1990) 281.

[16] N. Berkovits, "The Ten-Dimensional Green-Schwarz Superstring is a Twisted NeveuSchwarz-Ramond String", Nucl. Phys. B420 (1994) 332, hep-th/9308129;

N. Berkovits and C. Vafa, "On the Uniqueness of String Theory", Mod. Phys. Lett. A9 (1994) 653, hep-th/9310170;

N. Ohta and J. Petersen, " $N=1$ From $N=2$ Superstrings", Phys. Lett. B325 (1994) 67, hep-th/9312187.

[17] E. Verlinde and H. Verlinde, "Multiloop Calculations in Covariant Superstring Theory", Phys. Lett. B192 (1987) 95;

U. Carow-Watamura, Z. Ezawa, K. Harada, A. Tezuka and S. Watamura, "Chiral Bosonization of Superconformal Ghosts on Riemann Surface and Path Integral Measure", Phys. Lett. B227 (1989) 73.

[18] I. Antoniadis, E. Gava, K. Narain and T. Taylor, "Topological Amplitudes in String Theory", Nucl. Phys. B413 (1994) 162 hep-th/9307158;

M. Bershadsky, S. Cecotti, H. Ooguri and C. Vafa, "Kodaira-Spencer Theory of Gravity and Exact Results for Quantum String Amplitudes", Comm. Math. Phys. 165 
(1994) 311 hep-th/9309140;

M. Bershadsky, S. Cecotti, H. Ooguri and C. Vafa, "Holomorphic Anomalies in Topological Field Theories", Nucl. Phys. B405 (1993) 279.

[19] N. Berkovits, "Super-Poincaré Invariant Superstring Field Theory", Nucl. Phys. B450 (1995) 90.

[20] M. Bershadsky, A. Vaintrob and S. Zhukov, to appear.

[21] G. 't Hooft, "A Planar Diagram Model For Strong Interactions," Nucl. Phys. B (1974).

[22] S. Sethi, "Supermanifolds, Rigid Manifolds and Mirror Symmetry," Mirror Symmetry II, ed. B. Greene and S.-T. Yau, Int. Press 1997.

[23] I. Bars, "Free Fields And New Cosets of Current Algebras," Phys. Lett. B255 (1991) 353. 